\begin{tabular}{|l|l|}
\hline $\begin{array}{l}\text { 2. To: (Receiving Organization) } \\
\text { Distribution }\end{array}$ & $\begin{array}{l}\text { 3. From: (Originating Organization) } \\
\text { Engineering Testing } \\
\text { Laboratory }-82700\end{array}$ \\
\hline $\begin{array}{l}\text { 5. Proj./Prog./Dept./Div.: } \\
102250 / \text { B000 }\end{array}$ & $\begin{array}{l}\text { 6. Design Authority/ Design Agent/Cog. } \\
\text { Engr.: } \\
\text { Design Auth.: George Janicek }\end{array}$ \\
\hline
\end{tabular}

8. Originator Remarks:

For review and release.

4. Related EDT No.:

$N / A$

7. Purchase order No.:

$\mathrm{N} / \mathrm{A}$

9. Equip./Component No.:

N/A

10. System/Bldg./Facility: 200 Genera]

11. Receiver Remarks: 11A. Design Baseline Document? [] Yes [X] No

12. Major Assm. Dwg. No.:

N/A

13. Permit/Permit Application No.: N/A

14. Required Response Date: ASAP

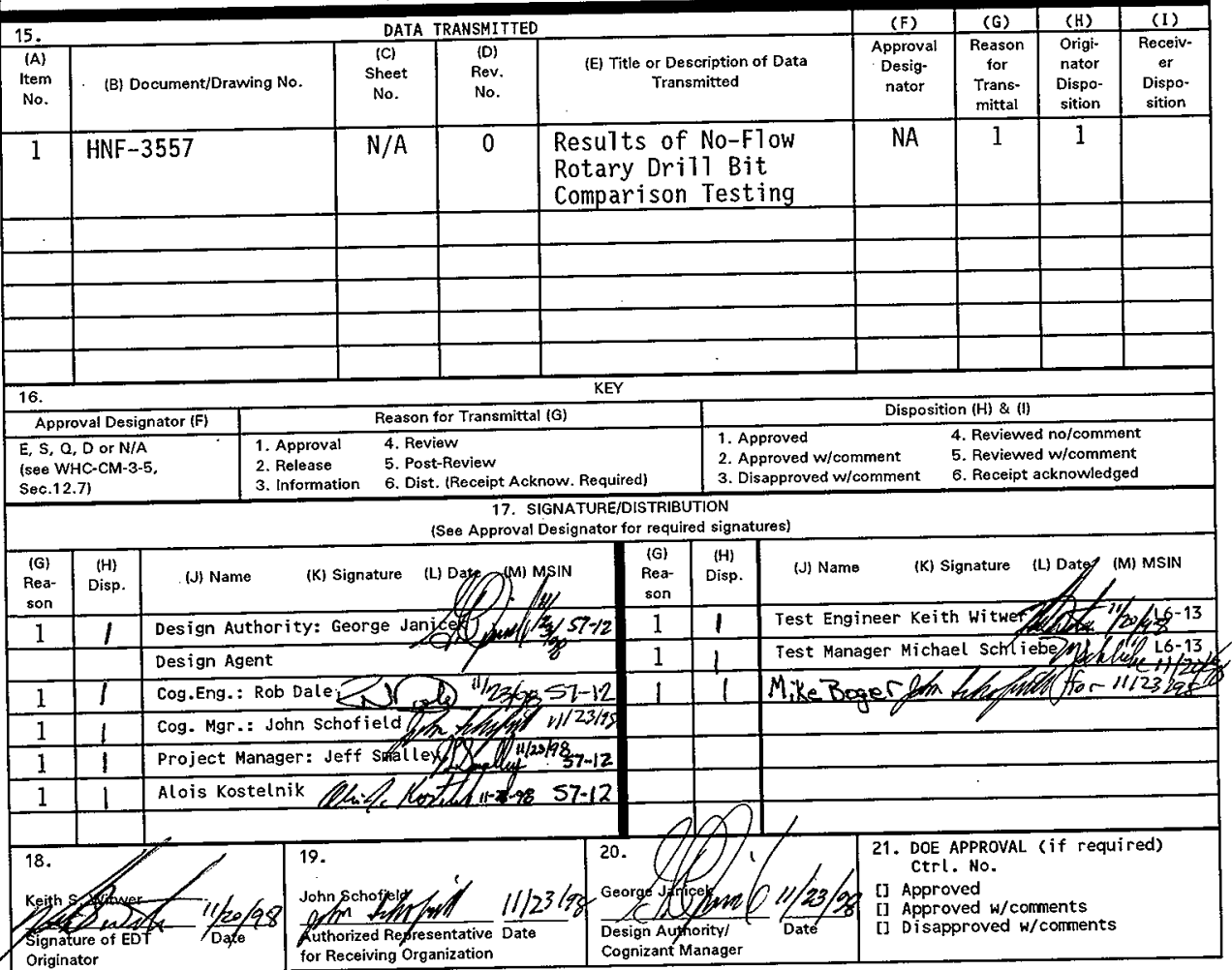

BD-7400-172-2 (05/96) GEF097 
HNF-3557, Rev. 0

\title{
RESULTS OF NO-FLOW ROTARY DRILL BIT COMPARISON TESTING
}

\author{
KEITH S WITWER \\ Numatec Hanford Corporation, Richland, WA 99352 \\ U.S. Department of Energy Contract DE-AC06-96RL 13200
}
EDT/ECN: 601070 UC: 2070
Org Code: $82700 \quad$ Charge Code: 102250/B000 / HNQ2033/
B\&R Code: EW3120074 Total Pages: 87

Key Words: No-Flow, Rotary, RMCS, Sampler, Drill Bit, ETL

Abstract: This document describes the results of testing of a newer rotary sampling bit and sampler insert called the No-Flow System. This No-Flow System was tested side by side against the currently used rotary bit and sampler insert, called the Standard Sytem. The two systems were tested using several "hard to sample" granular non-hazardous simulants to determine which could provide greater sample recovery. The No-Flow System measurably outperformed the Standard System in each of the tested simulants.

TRADEMARK DISCLAIMER. Reference herein to any specific commercial product, process, or service by trade name, trademark, manufacturer, or otherwise, does not necessarity constitute or imply its endorsement, recommendation, or favoring by the United States Government or any agency thereof or its contractors or subcontractors.

Printed in the United states of America. To obtain copies of this document, contact: Document Control Services, P.O. Box 950, Mailstop H6-08, Richland WA 99352, Phone (509) 372-2420; Fax (509) 376-4989.
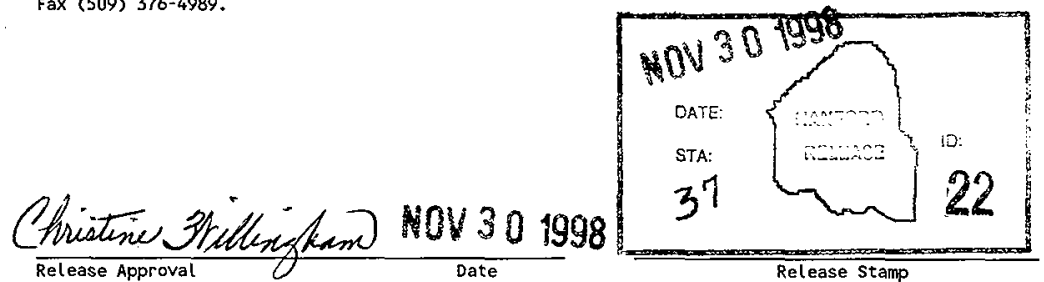

\section{Approved for Public Release}


RESULTS OF NO-FLOW ROTARY DRILL BIT COMPARISON TESTING

HNF- 3557, Rev. 0

October 1998

Keith S. Witwer

Engineering Testing Laboratory

Numatec Hanford Company

Richland, Washington 


\section{TABLE OF CONTENTS}

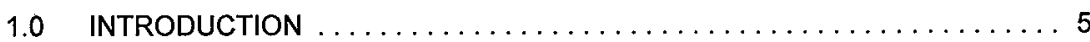

2.0 TEST OBJECTIVE $\ldots \ldots \ldots \ldots \ldots \ldots \ldots \ldots \ldots \ldots \ldots \ldots \ldots$

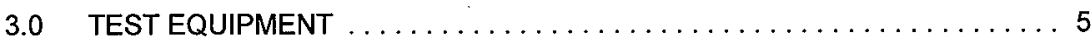

3.1 CORE DRILLING MACHINE $\ldots \ldots \ldots \ldots \ldots \ldots \ldots \ldots \ldots \ldots$

3.2 ROTARY DRILL BITS $\ldots \ldots \ldots \ldots \ldots \ldots \ldots \ldots \ldots \ldots \ldots \ldots \ldots \ldots$

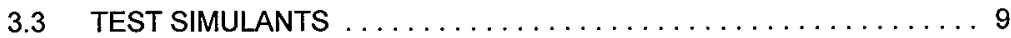

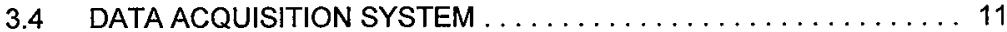

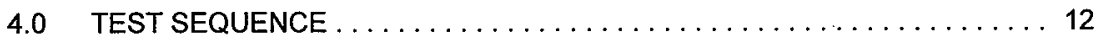

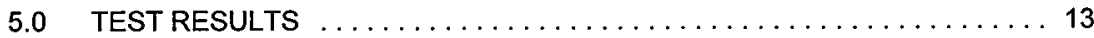

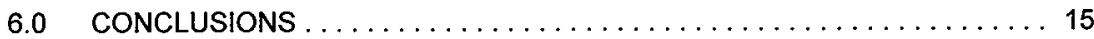

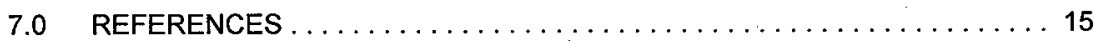

APPENDIX A - EQUIPMENT CALIBRATION DATA $\ldots \ldots \ldots \ldots \ldots \ldots \ldots$

APPENDIX B - CONTROLLED LOGBOOK NOTES $\ldots \ldots \ldots \ldots \ldots \ldots \ldots$

APPENDIX C - DAS RAW DATA FILES $\ldots \ldots \ldots \ldots \ldots \ldots \ldots \ldots \ldots \ldots \ldots$ 


\section{LIST OF FIGURES}

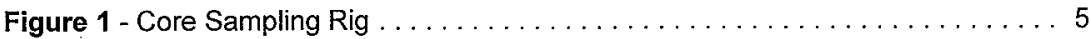

Figure 2 - Standard \& No-Flow Rotary Bits and Inserts $\ldots \ldots \ldots \ldots \ldots \ldots \ldots$

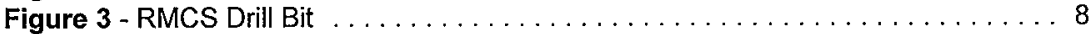

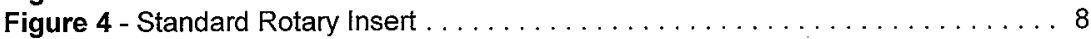

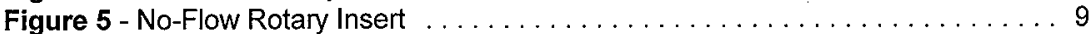

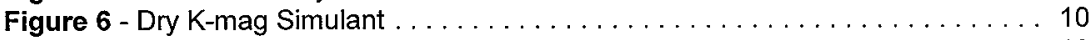

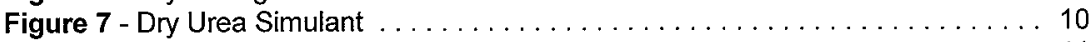

Figure 8 - Dry Sand Simulant . . . . . . . . . . . . . . . . . . . . . . 11

Figure 9 - Sample Recovery Comparison - No-Flow vs. Standard System . . . . . . 14

\section{LIST OF TABLES}

Table 1 - Bit Recovery Comparison . . . . . . . . . . . . . . . . . . 13 


\section{RESULTS OF NO-FLOW ROTARY DRILL BIT COMPARISON TESTING}

\section{$1.0 \quad$ INTRODUCTION}

Side-by-side sample recovery comparison testing was done during September 1998 between the rotary drill bit currently used for field sampling operations and a newly designed rotary mode drill bit. The rotary drill bit currently used for core drilling operations on the Hanford site (Longyear Inc. P.N. 100IVD/8) and its corresponding sampler insert, herein called the "Standard System", allows a significant amount of nitrogen purge gas to enter the center sample core rather than be directed out only the purge gas holes on the cutting face of the bit. This misdirected purge gas flow can disturb a cored sample such that the quantity of a sample recovered could be significantly reduced in certain waste forms. The new bit (Longyear Inc. P.N. 100IVD/9) and its associated sampler insert design, herein called the "No-Flow System", incorporates a leak proof seal between the sampler insert and the inside surface of the drill bit. This leak-proof seal prevents the purge gas from directly entering the center sampler hole and should allow for better sample recovery and better sample integrity.

\subsection{TEST OBJECTIVE}

The testing described herein was intended to demonstrate improvements (if any) in sample recovery with the No-Flow System over the Standard System while sampling various "hard to recover" simulants.

\subsection{TEST EQUIPMENT}

\subsection{CORE DRILLING MACHINE}

The drilling machine used was a Longyear Model $34^{1}$. This testing drill, in its basic form, is the same type of drill installed on the core sampling trucks for field operations.

The drilling machine is shown in Figure 1. 
Figure 1 - Core Sampling Rig

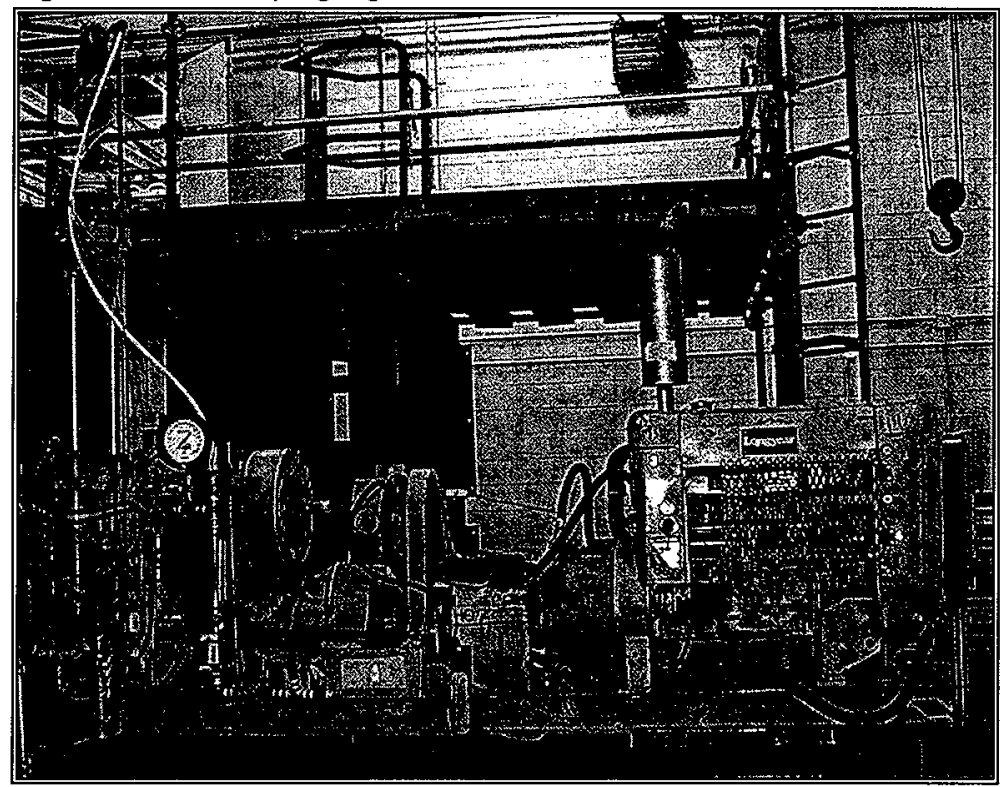

\section{$3.2 \quad$ ROTARY DRILL BITS AND INSERTS}

Drill bits used for this testing were Longyear part numbers 100IVD/8 (standard rotary bit) and 100IVD/9 (No-Flow rotary bit). The bits have the same overall geometry except for the inside sampler sealing surface. The standard bit has three equally spaced pins on the inner bottom surface that the sampler rests on while the No-Flow bit has a circumferentially flat, downward and radially inwardly sloping surface that the sampler rests on. A rotating mechanism, called a "sampler insert," screws into the bottom of a rotary sampler assembly and rests on the inner bit sealing surface. Each style rotary bit mates up to its own unique sampler insert. The standard sampler insert has a flat rotating surface that rests on the three pins mounted inside the standard bit. The No-Flow insert has a downward sloping circular surface set at a slightly sharper angle than the inside bit surface. This provides a sharp-edged sealing surface. Figure 2 shows the geometry of the two systems. Both style bits are composed of the same material composition - a proprietary sintered bronze and tungsten mixture. Each bit tested had a unique serial number and passed a receipt inspection before being used. 
Figure 2 - Standard \& No-Flow Rotary Bits and Inserts

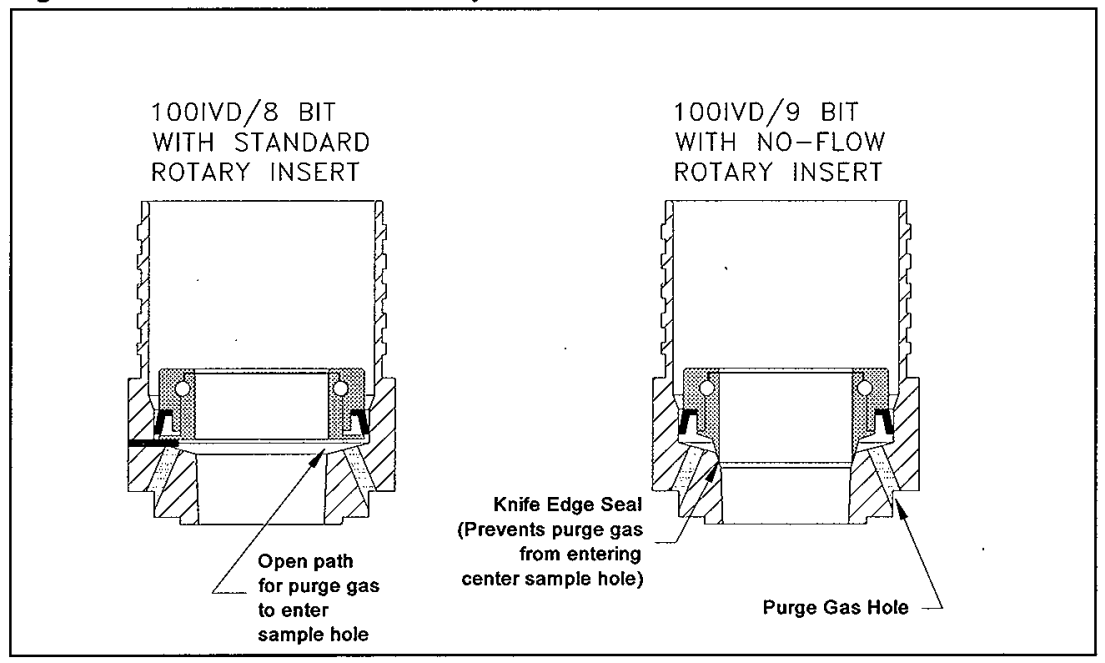

A photo of the standard RMCS (100IVD/8) rotary drill bit is shown in Figure 3. Figures 4 and 5 show photographs of the Standard and No-Flow inserts respectively. The photograph of the Standard insert is shown with the chevron seal while the NoFlow insert is shown without the seal installed. Note that the No-Flow insert would normally have a chevron seal fused to the insert for field sampling operations, but for this testing it was not used. This did not affect any tests results since the seal acts primarily as a fluid back-flow preventer which was not needed here since the simulants contained no free liquids. 
HNF- 3557

Rev. 0

PAGE 8

Figure 3 - Standard RMCS (100IVD/8) Drill Bit

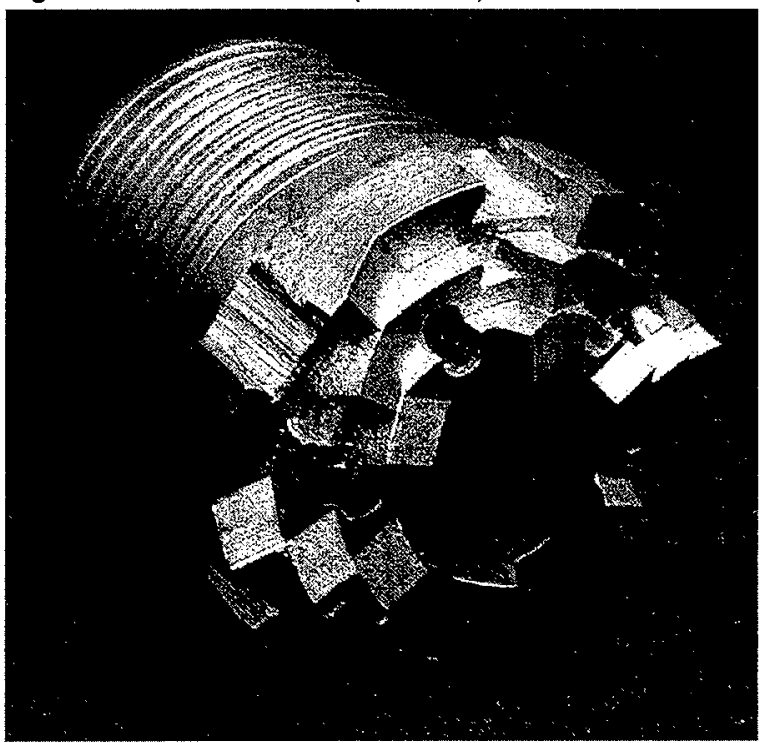

Figure 4 - Standard Rotary Insert

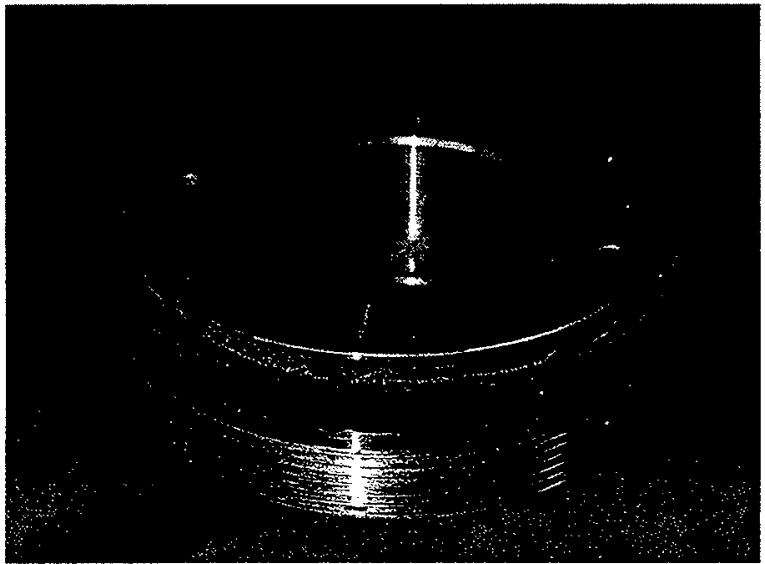


Figure 5 - No-Flow Rotary Insert

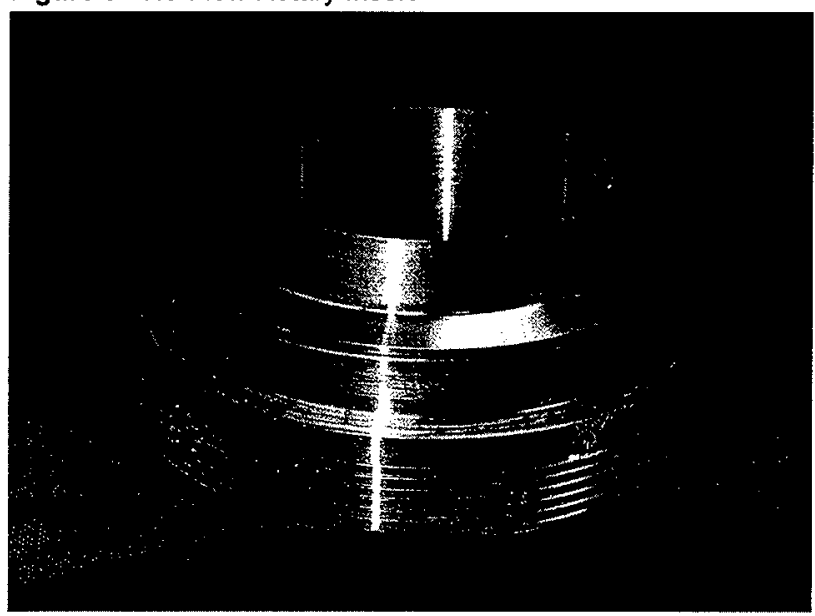

\subsection{TEST SIMULANTS}

Three nonhazardous materials were chosen as test simulants: sulfur Kmag [potassium magnesium sulfate $-\mathrm{K}_{2} \mathrm{SO}_{4}{ }^{*} 2\left(\mathrm{MgSO}_{4}\right)$ ], urea $\left(\mathrm{NH}_{2} \mathrm{CONH}_{2}\right)$ and common sand. The sand and the urea were tested both wet and dry. The Kmag was tested only dry since previous testing showed it too easy to sample when wet. The simulants were prepared as "wet" by adding water to a container already full of dry simulant until the simulant was saturated. The water was then allowed to completely drain out the bottom of the container a few minutes later. The dry simulant was used "as received" from the manufacturer/vendor. Wet and dry combinations of the three materials gave a total of five simulant combinations for testing.

Figures 6,7 and 8 show samples of each of the simulants. 
HNF- 3557

Rev. 0

PAGE 10

Figure 6 - Dry K-mag Simulant

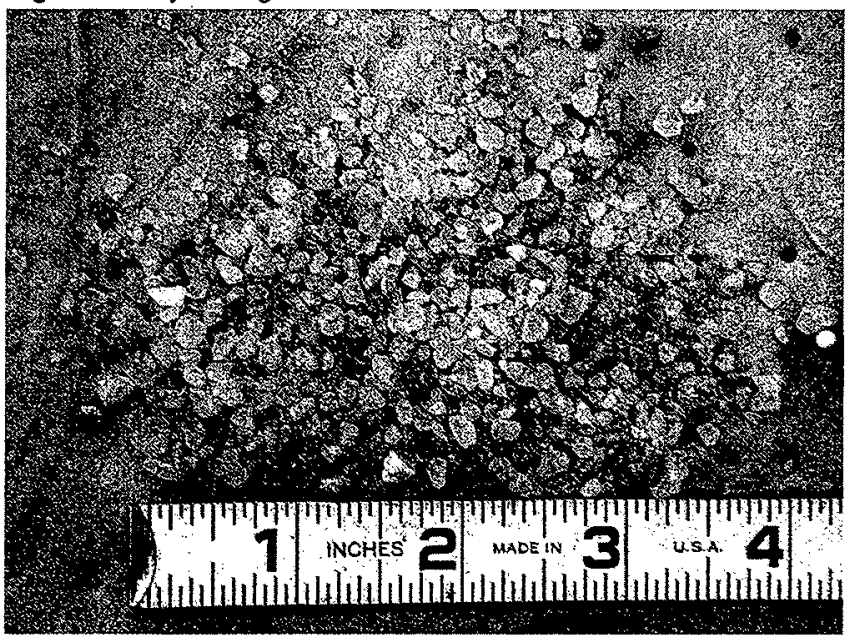

Figure 7 - Dry Urea Simulant

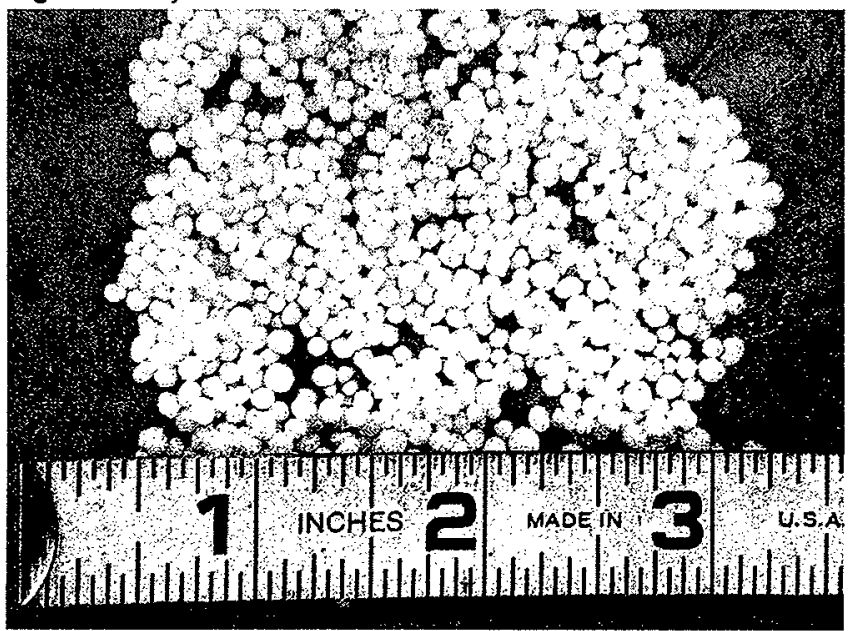


Figure 8 - Dry Sand Simulant

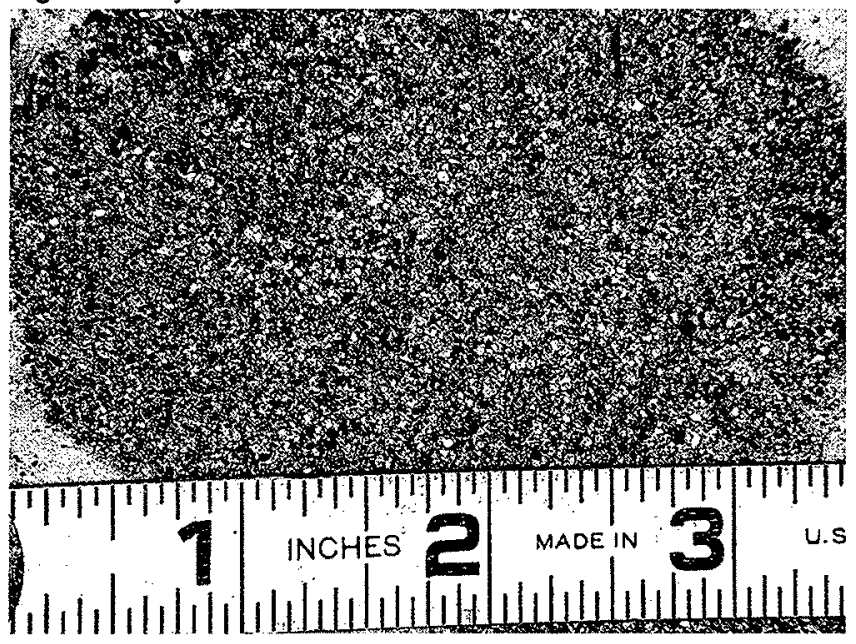

\subsection{DATA ACQUISITION SYSTEM}

A data acquisition system (DAS) was used to record pertinent drilling parameters. These drilling parameters included: purge gas indicated flow rate, purge gas pressure, purge gas temperature, drill bit rpm, drill bit down force, drill bit depth, drill bit penetration rate and elapsed time. Each sensor providing information to the DAS was under a current laboratory calibration or was verified as accurate using NIST traceable secondary calibration sources (see Appendix A). Note that most equipment items associated with the Rotary Mode Core Sampling (RMCS) system uses the English system of units. This includes items such as sampler length, diameter, bit diameter, etc. Subsequently, many items recorded during testing and described in this test report are given in the English system for clarity. 


\subsection{TEST SEQUENCE}

Each simulant tested was contained in a 24 " tall by 16 " diameter container with the simulant level set to at least 22" deep. The following list describes steps taken to perform a test.

1) A sampler, with the appropriate insert, was loaded into a standard core barrel that was then attached to the drill string mounted in the drill chuck of the drill machine.

2) The drill string/core barrel was then lowered until the bit face just touched the simulant surface.

3) The grapple assembly was then lowered down from an overhead crane hook, inside the drill string, onto the pintle rod, and secured to the drill frame. This depth became the zero point for the sampler and was electronically set as 0 " on the data acquisition system.

4) Static downward force (750 lbs.) and purge gas (30 SCFM) was set.

5) The drill string/bit was then set to rotate at 55 RPM.

6) Downward travel and DAS recording were started simultaneously.

7) The drill was allowed to penetrate the simulant for 19 inches and then downward motion was stopped. The grapple assembly was then raised, which triggered the sampler valve - if it had not already automatically closed by passing the $19^{\prime \prime}$, travel point.

8) The sampler was removed from the core barrel and weighed after each test. The pretest weight was subtracted from the post test weight giving the total sample weight. This information was recorded in a controlled logbook (HNF-N-23-1) 


\subsection{TEST RESULTS}

Of the five possible simulants, only three were found useful for comparison testing. Wet sand, dry urea, and dry K-mag gave measurable results. Five tests with each of the two bit/insert combinations were done in the wet sand simulant and six tests were done with each bit/insert combination in both the dry urea and dry K-mag. The other two simulants, dry sand and wet urea, gave such low recoveries with each bit/insert combination that recovery comparison was not measurable (i.e., one or two grams of simulant at most).

It was noticed during testing that the maximum down force developed was around $200 \mathrm{lbs}$ - although the drill was preset to allow up to $750 \mathrm{lbs}$ down force. The drill penetration rate was limited to approximately 140 inches/minute, in each of the simulants tested, by the capacity of the hydraulic system. More down force could have been developed by the simulant if the penetration rate were higher.

In addition, it was also noticed that with purge gas flowing and without bit rotation, the simulants would "fluidize" beneath the bit and the bit would penetrate fairly well. Without purge gas and likewise without bit rotation, the applied down force would increase rapidly as the bit was lowered - and $750 \mathrm{lbs}$ would be developed within 4-6 inches, but the bit would not continue to penetrate. This information is pertinent in that it reveals the inadequacy of a Push-Mode Core Sampling system (which does not have the ability to use purge gas) for drilling in some, otherwise easy to sample, granular materials.

Table 1 shows the average recovery for each bit/insert combination in each of the three useful simulants. Three columns for each bit/insert combination describe the test results. The first column "Avg. Qty. Recovered" shows the mean average of all the tests runs in a particular simulant with a particular bit/insert. The second column "Percent of full sampler" shows what percentage of a full sampler the "Avg. Qty. Recovered" was. (Note that the recovery percentage is based on the mass of a $300 \mathrm{ml}$ volume of the simulant.) The third column shows the consistency of the test results by showing the standard deviation of the specific group of tests. Raw test data from the data acquisition system is included in Appendix $\mathrm{C}$.

Table 1 - Bit Recovery Comparison

\begin{tabular}{|l|c|c|c|c|c|c|}
\hline \multirow{2}{*}{} & \multicolumn{3}{|c|}{$\begin{array}{c}\text { Standard System } \\
\text { (P.N. 100IVD/8) }\end{array}$} & \multicolumn{3}{c|}{$\begin{array}{c}\text { No-Flow System } \\
\text { (P.N. 100IVD/9) }\end{array}$} \\
\cline { 2 - 7 } & $\begin{array}{l}\text { Avg. Qty. } \\
\text { Recovered } \\
\text { (grams) }\end{array}$ & $\begin{array}{c}\text { Percent } \\
\text { of full } \\
\text { sampler }\end{array}$ & $\begin{array}{c}\text { Standard } \\
\text { Deviation } \\
\text { (grams) }\end{array}$ & $\begin{array}{c}\text { Avg. Qty. } \\
\text { Recovered } \\
\text { (grams) }\end{array}$ & $\begin{array}{c}\text { Percent } \\
\text { of full } \\
\text { sampler }\end{array}$ & $\begin{array}{c}\text { Standard } \\
\text { Deviation } \\
\text { (grams) }\end{array}$ \\
\hline Dry K-mag & 129.3 & 32.4 & 50 & 207.8 & 52.1 & 34 \\
\hline Dry Urea & 55.7 & 24.6 & 7.1 & 98.6 & 43.5 & 11.2 \\
\hline Wet Sand & 32.4 & 8.1 & 15.2 & 95.1 & 23.8 & 11.2 \\
\hline
\end{tabular}


Notice in Table 1 that in each simulant the No-Flow bit demonstrated higher recovery and better consistency of test results. In terms of "Avg. Qty. Recovered," the NoFlow out-performed the Standard bit as follows:

\section{- Dry Kmag $-61 \%$ better recovery \\ - Dry Urea - $77 \%$ better recovery \\ - Wet Sand $-194 \%$ better recovery}

The chart in Figure 9 graphically represents the percent recovery (of a $300 \mathrm{ml}$ sampler) for each test in the three simulants. In the chart the samples are shown with the highest recovery to the left for clarity. "This is shown as Sample Number 1 although this is not necessarily the order in which the sample was taken during testing. Note that the NoFlow tests are shown as cylinders and the Standard tests are shown as rectangles. In addition, the simulant types are differentiated on the chart using unique surface textures.

Figure 9 - Sample Recovery Comparison: No-Flow vs. Standard System

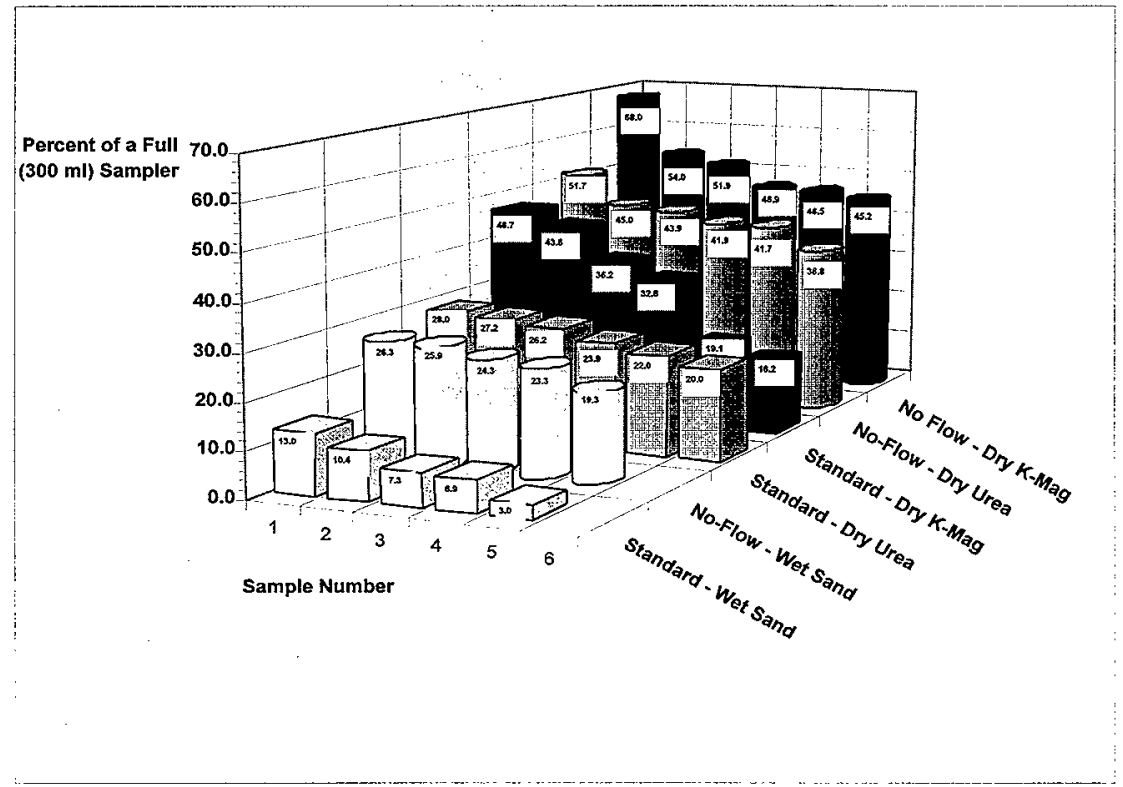

Any effect the No-Flow bit might have on sample integrity (how well the core stayed intact and realistically represented a particular depth segment) was not measured since 
incremental layering in the preparation of the simulant mixtures was not done. One could assume that sample integrity would improve with the No-Flow bit due to the geometry of the sealing surface and the subsequent redirected flow pattern.

\subsection{CONCLUSIONS}

The test results show that the No-Flow System outperforms the Standard System in each of three different simulants. Although these simulants may not perfectly replicate material found in Hanford waste tanks, they do represent hard-to-recover materials and differ from each other in several physical attributes - such as particle size, shape, density and water content:

For solid, easy-to-recover simulants, the Standard and No-Flow System would recover roughly equivalent samples since the purge air flow pattern would not disturb the solid core. Were the sample to break up during recovery, the No-Flow would perform better since it would have less tendency to disrupt the sample.

The No-Flow system forces all the available purge gas out the purge gas holes rather than allowing some to escape into the center sample hole area. This allows a higher velocity purge flow which should help scavenge the cuttings away from the bit face more effectively. Also, the purge holes are more likely to remain free of blockage since the path between the sample core and the purge path is closed. Examination of the drill bit after testing with the Standard System showed that in several instances the purge holes were either partially or completely blocked. Blocked purge holes decrease bit penetration efficiency since particles are not removed from the bit cutting zone.

\subsection{REFERENCES}

1. HNF, 1997, HNF-N-23-1, Laboratory Logbook for Engineering Testing Notes, Keith Witwer, Numatec Hanford Corporation, Richland, Washington. 


\section{APPENDIX A - EQUIPMENT CALIBRATION DATA}

\section{Equipment Calibration Data}

\begin{tabular}{|c|c|c|c|}
\hline मltem & Hanford Std Lab & Range/Accuracy & Cal Expiration \\
\hline $\begin{array}{c}\text { Omega CL505A Temperature } \\
\text { Calibrator }\end{array}$ & $750-13-55-002$ & $+1-0.5^{\circ} \mathrm{C}$ & $6 / 15 / 99$ \\
\hline $\begin{array}{l}\text { Celesco PT101-40A - Depth } \\
\text { Indicator }\end{array}$ & ETL Lab Cal & $\sim 24 "+/-1 / 16^{\prime \prime}$ & Cal/Accur chk as req'd \\
\hline $\begin{array}{l}\text { Toledo/Mett Mdl } 8140 \\
\text { Platform Scale }\end{array}$ & $750-66-01-004$ & $0-2000 \mid b(C a l) \pm 2 l b$ & $12 / 21 / 98$ \\
\hline Cole-Parmer Flowmeter & $N / A$ & 4-50 SCFM +/- 1 SCFM & $1 / 23 / 99$ \\
\hline Type K Thermocouple & ETL Lab Cal & $0-300^{\circ} \mathrm{C} / \pm 2^{\circ} \mathrm{C}$ & Cal/Accur chk as req'd \\
\hline Purge Gas Pressure Gage & $752-31-04-085$ & $+/ 1 \mathrm{PSI}$ & $12 / 30 / 98$ \\
\hline
\end{tabular}


HNF- 3557

Rev. 0

PAGE 17

APPENDIX B - CONTROLLED LOGBOOK NOTES 


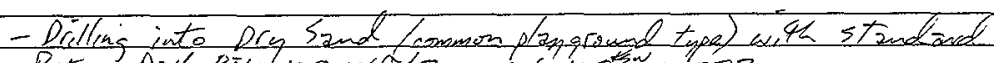
Rotarn Dill Bit $100160 / 8$, sw toow 1072 ?

- 250 lhe, 55 EPM, 30 SCFM

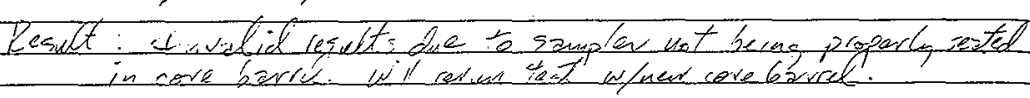

-Test B10772

- Repeat of last test-dilling into diry sand of standiand cutarg lit (100100/8) 5010772

$=750 \mathrm{H}, 55 \mathrm{RPM}, 30 \mathrm{SEEM}$

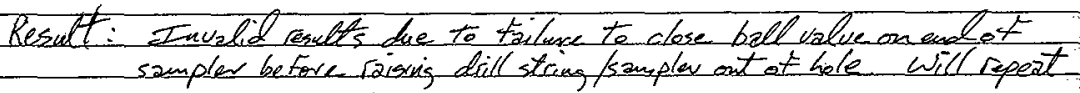

- Test c10272

- Repeat of last test Drilling into Lry sand w/standasd totary bit $(100100 / 2) \leq N 10772-(\operatorname{san} 2$ lev \pm 4$)$ $-75015 \mathrm{~F}, 55 \mathrm{RPM}, 30 \mathrm{SCFM}$ (Wo pinte rod 1 insert) Zesult: 6ood test Total wt of samplew betare test $=4553$ qrams Total wt of sampler after test $=4553$ grams (w/sand sample)

- Note that downtorce registers litte or noling on floes scale during

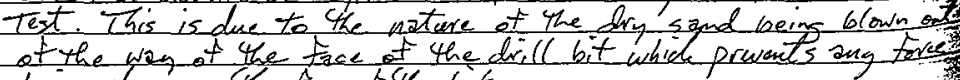
being shown on the fice ot the bit.
4

Cest F E Elo7 - Drille. SN $-75016 f$ 呇 Res dt: $\omega_{0}$ Cx:

8 . 4. Note: Test c e 107 - Dirille. rotar. 7501 


\section{Test 010722}

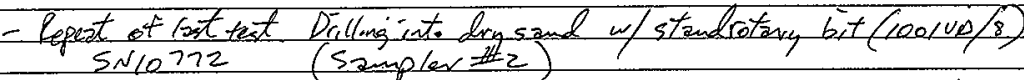

-750 (b), $55 \mathrm{RPM} 30 \mathrm{SCEM}$

\$ to pintie rod. insert

Result: Good test. Total wt of samplex Getore =

rotal wt of sampleveffer $=4269.7 \mathrm{grams}$

Test $=10772$

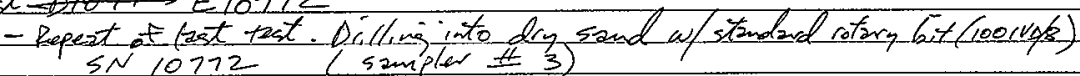

- 750 lbf, LS RPM, 30 SCFM

Result: beel tat

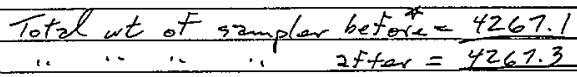

Test F10172

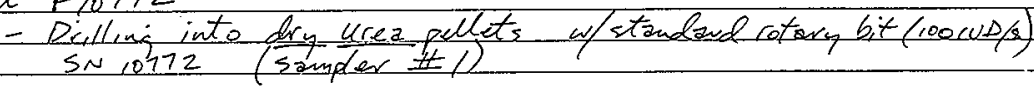

$-75016+, 55 R P M, 305 C F M$

Reselt: 6ood Test sampler wt Getore: 4410.0 " $"$ aftar. 4471.8 Note: Liter the sand, aedounctore registered on the floor scale (see Tast Test c c $1077 z$

- Drilling into dry urea pellets (Repeat of last test) Using standzond rotary bit (100100/8) SN10772 (sampler 12 ) $-75016,55 R P M, 30$ SCEM

Resut: Good Tat Sampler wt before $=4433.6$ after $=4569.5$ ksw $\rightarrow 4487.7$ 
HNF- 3557

86

- Test $H 10772$

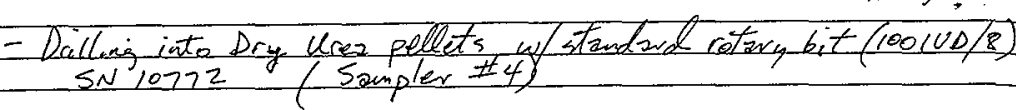

$-75016 F, 55 R P M, 30$ SCFM

Result: Bastrest Samplec wt atore 4717.5 grams

- stuck spring-release mechanism prevented boll walue - Test I10772

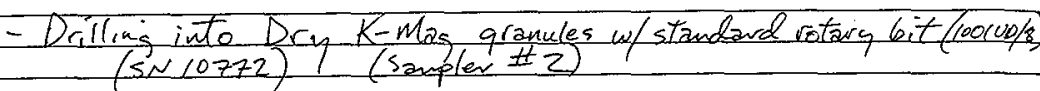
$(5 N / 0772)$ (Saxpler $t$

Resuet: Good Test

Samplear wt betore test $=\frac{4433.6}{4498}$

Test T10772

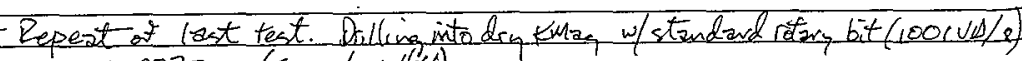
SN 10772 (szimplen 14 ).

$-75016 \neq, 55 \mathrm{RPM}, 30 \mathrm{SCFM}$

Rerult: Good test Somplear wt betork: 4717.5 ar

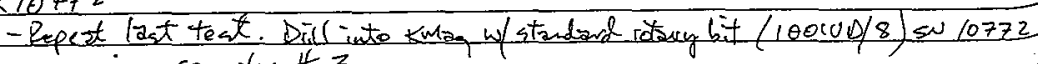
using samplew 3

-750 bF, $55 \mathrm{RPM}$. 30 SCFM

Result. Baplest-

Euraged dsill itring up

betare clesing ball vave-will resem test.
Somples wt betare $=-1 / \sigma$

1) after $=\quad / / 6$
-Test $A-N F I$

- Divil inteo sed

$-75016 F$

Eesult: Coce

Test B-NF - Dillinto sen

-750 16

Result: Cos

$-7 e s t C-n$

$\frac{- \text { Repert } 1}{5}$

$-75016$

Result: 


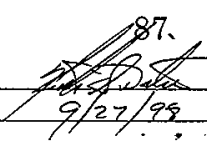
-750 bF, $55 \mathrm{RPM}, 30 \mathrm{scFM}$
Result: $600 d$ Test Wt sampler BEFORE $=4410$ grams
". AFter $=4583.9$

\section{Test $A-N F I$}

- Diul into dry K-mlaq w/No Flow Insent $\$$ Rotary Drill Bit $100100 / 9$

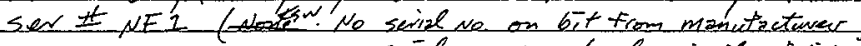
se a new suial ve wes placed on inside ot sit "NFi")

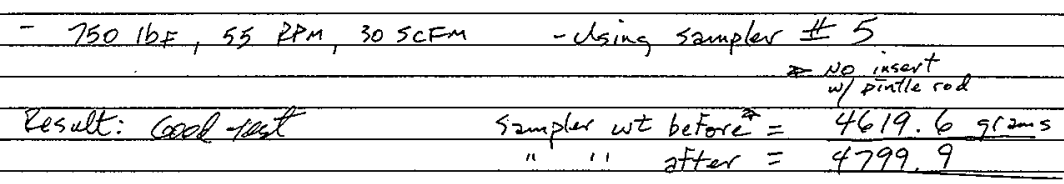

Test $B-N E I$

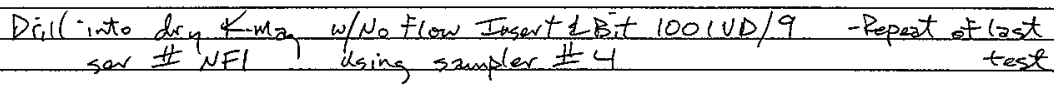
-250 bF, $55 \mathrm{RPM}, 30$ SCFM

Result: Good Test $\begin{aligned} \text { Senplewt betere } & =4717.5 \text { ar } \\ \text { in in viter } & =4924.7\end{aligned}$ Test C-NFI

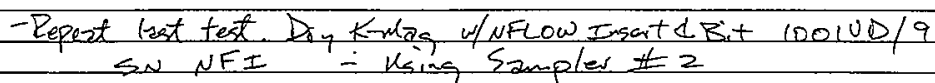
-750 ibp, $55 \mathrm{RP}, 30$ SCFM

Result: $\begin{aligned} \text { Samples wt betere } & =4433.6 \\ \text { i. } 11 \text { after } & =4619.3\end{aligned}$ 
88

Test D,NEI

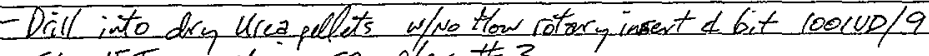
su NEI - Using sampler \pm 3

$-75016 F, 55 \mathrm{RPM}, 30 \mathrm{SCFM}$

- Result: Goed test

- Test E-NFI

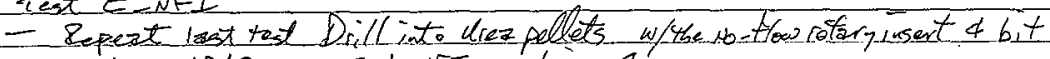
$100100 / 9-5 N$ NEI - Using 1

-750 ibF, $55 \mathrm{RPM}, 30$ SCFM

- Result:Good test
Sampler wt before $=4434.6$ grams after $=4529.1$ grams
- Test F NFI

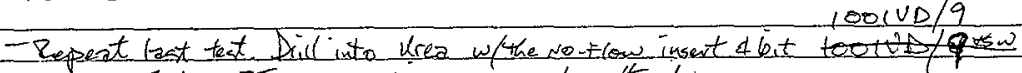
$S N=N E I$ - Using sampler II

$-75016=, 55 \mathrm{RPM}, 30 \mathrm{SCFM}$

- Result:-6od fent
Sampler wt before $=4410$ grams
$=$ Text I_N

- Repeat. Q

$-750(b+1$,

- Eosult:

Text G-NFI

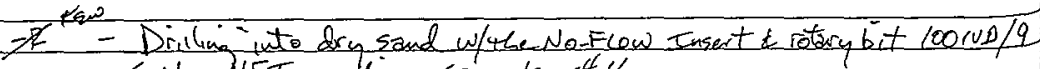
$S N-$ NFI lising sampler 44

- 750 ib, 55 RPM 30 SCEM

Result Good test

Sampler wt betore $=47 / 7.5$

1 i. after $=4717.5$ 
HNF- 3557

- Test H-NFI

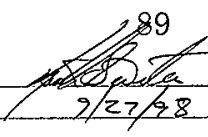

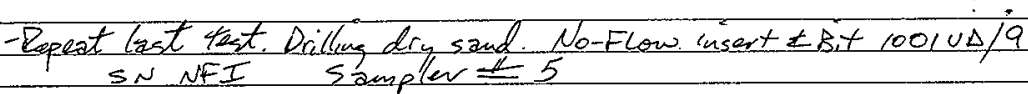

-750 b 5 , $55 \mathrm{RPM}$. $30 \mathrm{SCFM}$

- Result: coos tred

samplar wt betore $\frac{46 / 9.6 \text { grms }}{4620.6 \text { grams }}$
in after

TeSt I NEI

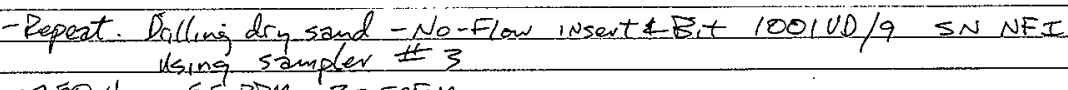

$-150 \mathrm{ch}, 55 \mathrm{RPM}, 30$ SCFM

- Rosult: Good test

Sampler wt betore $=4434.6$ grams
in atter $=445.4$ grams

Then $18 \mathrm{M} 10772$

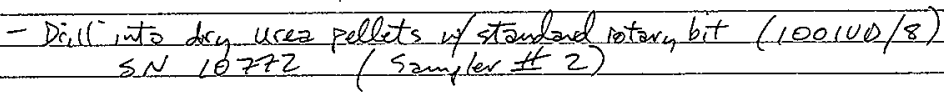

- $750 \mathrm{Cb}, 55 \mathrm{RPM}, 30$ SCFM

Reselts:

Sampler wt before $=4433.6$ grams 
HNF- 3557

90

Photos of similants used.
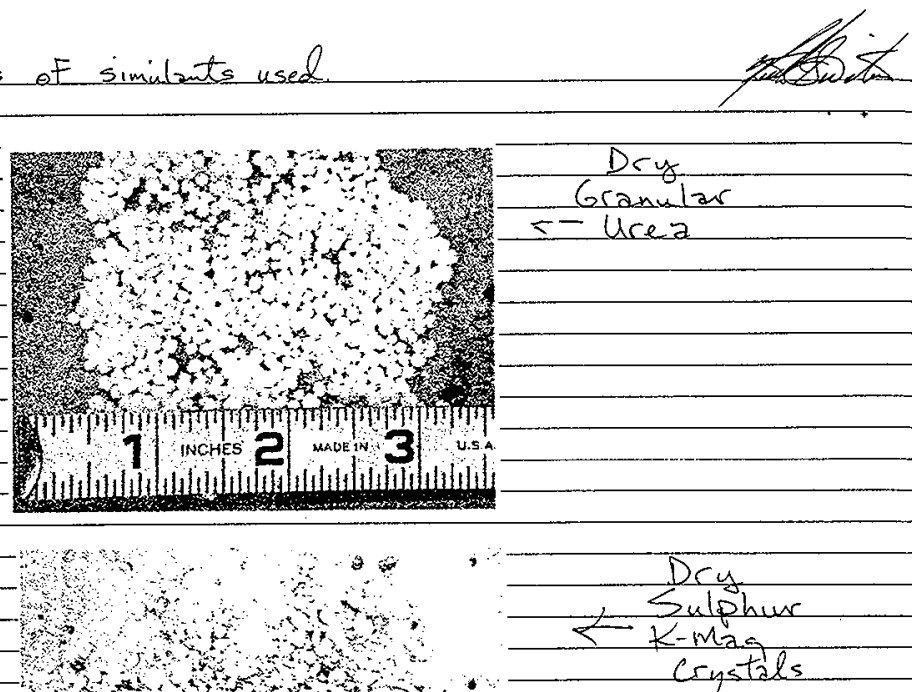

$$
\begin{aligned}
& \text { Dry } \\
& \multirow{2}{*}{\text { Sulphur }} \\
& \text { Crystals }
\end{aligned}
$$

Test: $010^{-}$

- Drill is - Sami $-250$

Reswit:
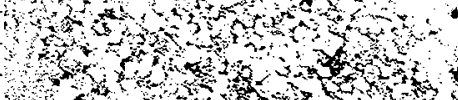

ond $x^{2}+2$

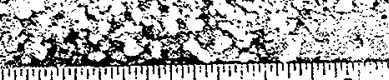

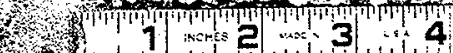

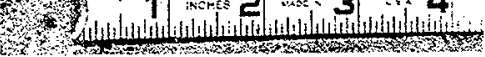
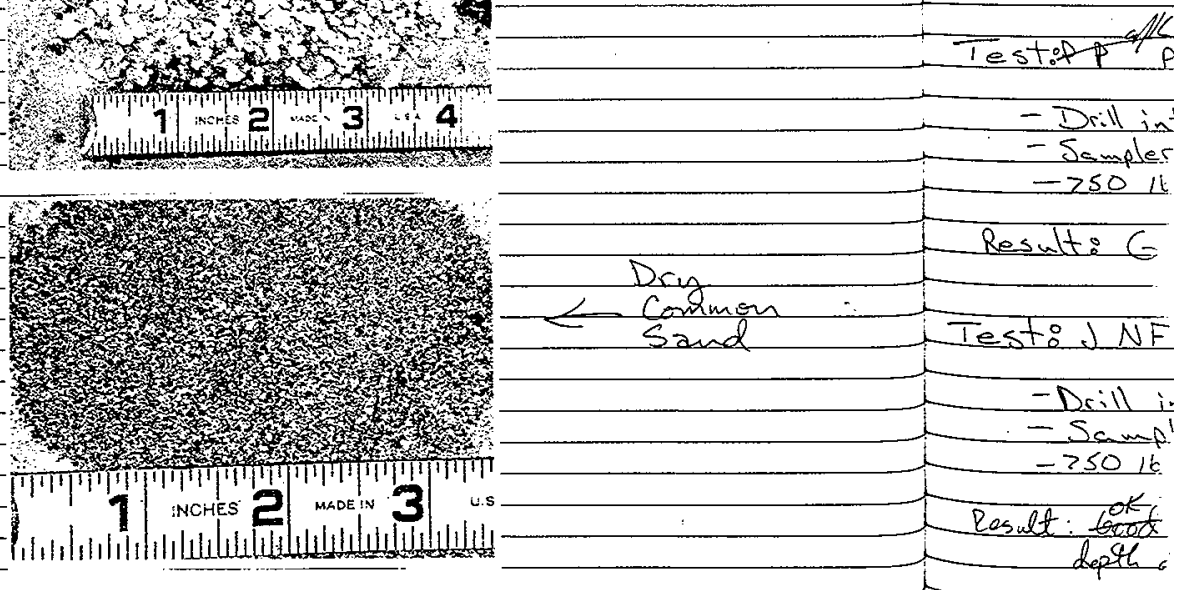
Test: N 10772

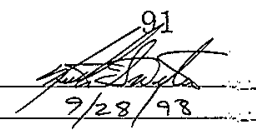

Drill into diy Urea pellets $w /$ standard rotary bit $(100 I \cup 0 / 8)$ (Sampler It 5$)$ $-750 \mathrm{lbF}, 55 \mathrm{RPM}, 30 \mathrm{SCFM}$

Result: Good Test Sampler wt before 4619.6 grams $\therefore$ "after 4665 ..

Test: 010722

- Drill into doy urea pellets w/ standadd cotary bit (100TU0/8) - Samples 2

$\rightarrow 50$ ibf, S5RPM, 30 SCFM

Result: Cooed Test Sampler wt before 4434 grams
"

Test:p plll $\rho, 0>>2$ - Drill into dry urea pellets w/ standard rotary bit (100IJD/8)

-250 l6f, SSRPM, 30 SCFM

Result: Geod Tost Sampler wt before 4717.5gans

Test: JNFI

- Drill into dry urea pellets w/ No Flow bit of insect (100IVD/9)

- Sampler 3

$-75016 \mathrm{f}, 55$ RPM, 30 SCFM

Result: ok ksw test although computal $i$ le yas not recorded (maltunction) and depth of sample was a little short showd probalely not use Ghes diata.

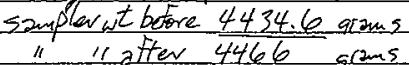


92

Test KNEI

- Drill into dyg urea pellets using No-ELow Nsert and bit $100100 / 9$ using samplev \#1 $-750 \mathrm{lbF}, 55 R P M$, SO SCFM

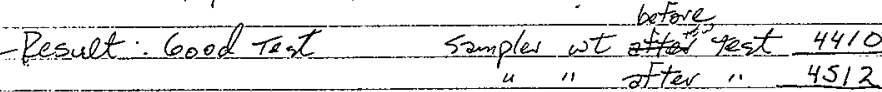

Test P NFI $-D r_{1}(\log$ $-2500$ Result very good

- Test $L$ NFI - Dill into dicyulrea pellets using No-Flow waERT \& bit $100100 / 9$

$-25016 \neq, 55 R P M, 305 \mathrm{cFM}$

- Result: Good test

Sampler wt before Test $\frac{4410}{4505}$ grams

-Test M NEI

- Drill into dry ucea pellets using no-flow unsent 46 t 100 rop/9

$-75012 \neq, 55 \mathrm{RPM}, 30 \mathrm{SCFM}$ Using-sampler $\# 3$

- Result: Good test

Sampler wt before $\frac{4434.6}{4518}$ srams

Tat $B / 077$

-Drilling - sn $1 c$

- Resudt:60

Test Q10712
- Dill
$-\quad$ sn
- Result:
Tsee no $=$ Test: 51072
- Drilling in
insert

Test N NEI

- Drilling into dry K-Wag using no-Flow insent $t$ bit $100 / v 0 / 9$

$-75016,55 \mathrm{RPm}, 30$ SCEM Using sampler I Z

- Result Good test Sampler wt batore $\frac{4434}{4621}$ grams

$-\operatorname{Resuts} 36$

- Test O NFI $=$ Drillininintodry K-mag using no-flow usent $46 i t$ looloD/9 Sampler wt betexe 4619.6 or

- Result: Good ted 
Test $P$ NEI

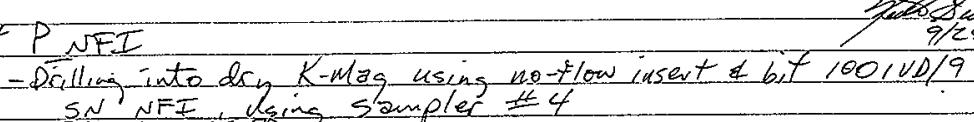
-750 (bF, $55 \mathrm{RPM}, 30$ SCFh

Result: Good test very good recovery (?) sampler wt betore 4717.5 grams 4989 grans.

\section{$=$ Test $Q 10772$}

- Diclling into dry K-Mar using sTandard rotary bit $1001 \mathrm{VD} / 8$ - SN 10772 , Using Sampler \#4; $75016 \neq, 55 \mathrm{RPM}, 30 \mathrm{SCF}$

- Result: Good Test Pser nete below.

sampler wt betore 4717.5 grams " affer 4904

Test $R \quad 10>72$

- Drilling into diy K-magusing standard ritary bit $100 \mathrm{rv0} / 8$ - sn 10222 , using sampler $5,75016 F, 55 R P M, 30$ SCFM

- Result: 600 d Test-See hotebelow sampler ut before 4619.6 grams

-Test: $5(0)>2$

- Drilling into dry Kmeg using standard rotary bit (100100/8) and - insert.

-Resultss lood test-Seenote sampler wt before $44 \mathrm{co}$ or

\# Hote: a fread Towards hifher recovery was noticed after test $Q 10772$. This was suspected to be caused by settlingroverectime. Test R10772 w2s run after the K-ulan, mix was renoued from the sample drum and then poured back in again. Hotice the skasp diop in sample wt: 76 grams in test R10772 is 186 grams in test $Q(0772$. The proces 2 wrs repeated ag a im betore test 510772 - same phenomena. Will semic the Org K-Mag betore each rum for consistemey on the Future 
- Test 710772

- Drilling into sulphur Kulag w/standard rotary bit $100110 / 8$ SN 10772 Using sampler $\pm_{2}$

-250 da 55 RPM, 30 SCFM

Result: bood Test sampler wt betore $\frac{4434}{4504}$ is

- Test Q-NFI

- Drillivg into Sulphar K-Man w/ No-Flow wSER \&Bit-100100/9 SN NEI Using sampler 3

$-75016,55 R P M-305 E M$

Result: cood rest

Sampler wt before 4434.6 ar

Test $R$ NFT

-Dirlling in Net urea w/No flow unsert $B$ Bt $=100110 / 9$ SN NEI Using samplar 22

-750 ib, 55 PBM, 30 SCEM

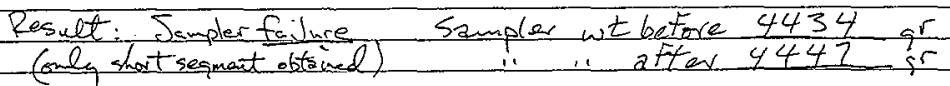
- Test S.NFI

- Drilling in wet urea w/No-Flow insert $/$ it $-100100 / 9$ SN NFI Using somplent/

$-750 \mathrm{~W}, \mathrm{S5} \mathrm{RPM}, 30$ SCFM

Rerult. "Goed" Tast

Sampler wt before 4410
" ater $4440=$ or

- Test TNFI

- Drillug in wet uced $\omega / \mathrm{N}_{0}$ - Flow inset $/ 6$ t $-100100 / 9$

-750 bi, $558 \mathrm{RM}, 30 \mathrm{SCFM}$

- Result: Samplertalure Samplor wt betere
$\frac{N / A}{N / A}$ ar
Test U NF Drilling: $-u_{\text {sing }} 5=$

-Result: 600d sample $A$ $=\operatorname{lis}^{t} V=N$

-Dictorisis $-1 \operatorname{sing}$ $-750+6 \%$

$-T e s t 4107$ - Dill usia $-75016 F_{T}$

Result: Sample trilure - the

$\frac{- \text { Test } \sqrt{107}}{- \text { Repest }}$ $-75016 \mathrm{~F}$

Rosult: $O E$ Test Probaldeqnota

Test wiot - Drilling - Samp $-750165$ - Result: 
- Test ULNFI Dcilling in wet Uerea mix using No-Elow bit/psest tow $10000 / 9$ - Using sampler \pm 1 -750 ib, 55 RPM, 30 SCFM

-Result: Good test but very swall sample. Maynot be suitatle sinnant. Sampler wt before will use standand bit to verity this -on next teit $=T_{12} t V=N F 5$

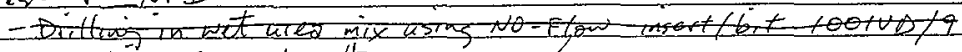
- ctsing sampter I

$-75016+55$ - 50 Sefa

- Test 410772

- Dill using standand bet 100 rvo/8 in wet ucea mix - 250 ibf, $55 \mathrm{RPM}, 30 \mathrm{scFM}$ - Sampler $z$

Result Samplerf Grapple tailure - Teat No Good. sample ut before 4434 gr - Test $\sqrt{10772}$

- Repeat et last test Dill using standad rotary bit $1001 \mathrm{v0} / \mathrm{s}$ en wet urea mix $-75016 \neq 55$ RPM, 30 SCFM $=5$-ampler 5

Result: Of Test Low volume sample. Sampler wt be fore 4619 Probaldy not a good simulant. sfter 4641 gr

$-\operatorname{les} w_{10} 272$

- Drilling into wet sand using standard cotary bit/insert $100100 / 8$ - Sampler \#3

$-75016 E, 55 R P M, 30$ SCFM

- Result:

Sampler wt betore 4434 sr 11. after 4446 ar 
HNF- 3557

ReV.0

PAGE 30

96

Test $\times 10772$

- Drillagi into wet sand w/standard rotary 100 rop/8

-750 (6), 55 RPM, 30 SCEM

Posult: Good test Sampler wt betore 4777.5 grows $\Delta \omega t=$

-Test $y 10772$

- Drillung into wet sand w/standard rotary 6 t $100100 / 8$ Using sampler \pm 1

-250 CSF, 55 RPM, 30 SCFM

- Result: Good Test

$$
\text { Sampler wt betore }
$$

\section{$4410=9 r$ 4462}

$\Delta w t$
$=$ Test U NFE

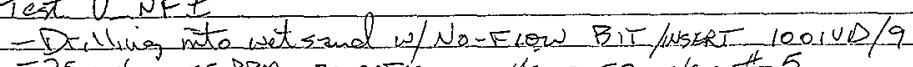

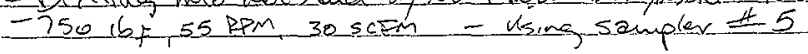

- Eesult 600d Test Sampler we betore 4619 ar
-Test W NFI

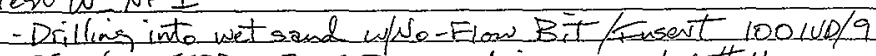

$-25016=, 55 \mathrm{RPM}, 30 \mathrm{SCFM}$ - Using sampler \#4

- Resulti Good Test samplear wt betore 4717.5 ar
-Test 21077 - Drilling its Using

$=750 \mathrm{lb} F, 55$

- Result: Go

Test

- Drilling int

- Reselt: 60
- Text Y NF

-Dcilling int Using $=$

$-750\left(b_{-F}, 5\right.$

-Resilt: Coost

\section{Test $x$ NEI}

- Drilling into wet sand who-Flos bit/unsert $1001 \mathrm{VD} / 9$

$-250 \mathrm{leF}, 55 \mathrm{RPM}, 30 \mathrm{ScFM}$ - Lsing sampler II 2

- Result: cood Test

$$
\begin{array}{r}
\text { Sampled wt betore } 4434 \\
2-2 \text { ater } 4531 \\
\Delta \omega t=
\end{array}
$$

$=$ Test Z.NFI

- Drilleng_int dsing sam

-750 (b), E

-Result: cood 
- Test $z 10772$

- Drilleng into wet szond w/standard rotareq bit 100 cuo/8 Using sampler \#4

$-75016,55 \mathrm{RPM}, 30 \mathrm{SCF}$

- Result: GoodTest

Sampler wt betore $4717 \leq$ or in 11 atfor 4745 or

Test AAlozzz $\Delta \omega t=27.5$

- Drilling into wet sand w/standard rotarn $6 i t$ it $10000 / 8$ Using sasspler 15 .5 750 bF, $55 \mathrm{RPM}, 305 \mathrm{CFM}$

- Reselt: Good Test Sampler wt betore = $\frac{4619}{4648}$

$$
\Delta w t=29.5 r
$$

Test Y NFT

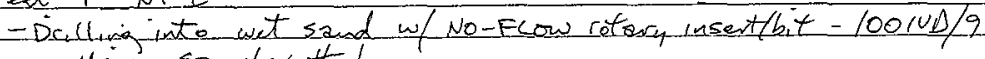
using sampler \pm 1

-750 \& $b, 55$ RPM, 30 SCFM

- Resent: Good test

Sampler wt Getare 4410 $\Delta \omega t=105$ ar

- Test Z NEI

- Dilling into wet sand w/No-Elow cotary insert $16 i t$ loolvo/9 Using sampler II 2

-250 ibF, $55 \mathrm{RPM}, 30 \mathrm{SCEM}$

- Result: $\cos 0 d$ test Sampler wt before 4434 ir $\Delta \omega t=27-g r$ 
PAGE 33

\section{APPENDIX C - DAS RAW DATA FILES}


100IVD/8_IN_DRY_SAND_SN_10772_750LB_55RPM_3OSCFM_TO_COMPARE_WINO_FLOW_INSERT TIME PURG_TC2 PurgePSI PurgeCFM DEPTH(IN) DwnFrc(lb) RPM Inches/Min

$\begin{array}{llllllll}05: 28: 10: 34 & 20 & -1.0 & 1.8 & 0.03 & 4 & 55.8 & -0.28 \\ 05: 28: 11: 33 & 20 & 21.4 & 59.0 & 0.43 & 4 & 55.8 & -0.54 \\ 05: 28: 12: 32 & 20 & 24.1 & 56.9 & 1.16 & 6 & 55.8 & -0.54 \\ 05: 28: 13: 31 & 20 & 24.1 & 56.5 & 1.98 & 7 & 55.1 & 13.28 \\ 05: 28: 14: 35 & 20 & 24.1 & 56.4 & 2.74 & 7 & 55.1 & 13.28 \\ 05: 28: 15: 34 & 20 & 24.1 & 56.3 & 3.55 & 7 & 54.7 & 14.36 \\ 05: 28: 16: 33 & 20 & 23.9 & 56.2 & 4.26 & 7 & 54.7 & 14.36 \\ 05: 28: 17: 38 & 20 & 23.9 & 56.2 & 5.06 & 7 & 54.7 & 13.15 \\ 05: 28: 18: 36 & 20 & 23.9 & 56.1 & 5.88 & 7 & 54.6 & 13.85 \\ 05: 28: 19: 35 & 20 & 23.9 & 56.1 & 6.58 & 7 & 54.6 & 13.85 \\ 05: 28: 20: 34 & 20 & 23.9 & 56.1 & 7.41 & 7 & 54.6 & 14.40 \\ 05: 28: 21: 33 & 20 & 23.9 & 56.1 & 8.14 & 8 & 54.6 & 14.40 \\ 05: 28: 22: 37 & 20 & 23.9 & 56.1 & 8.90 & 8 & 54.7 & 12.42 \\ 05: 28: 23: 36 & 20 & 23.9 & 56.0 & 9.69 & 9 & 54.7 & 12.42 \\ 05: 28: 24: 35 & 20 & 24.1 & 55.9 & 10.39 & 8 & 54.7 & 10.37 \\ 05: 28: 25: 34 & 20 & 24.1 & 55.9 & 11.18 & 12 & 54.6 & 10.37 \\ 05: 28: 26: 33 & 20 & 24.1 & 55.9 & 11.95 & 8 & 54.6 & 14.46 \\ 05: 28: 27: 37 & 20 & 24.1 & 55.9 & 12.70 & 8 & 54.7 & 14.46 \\ 05: 28: 28: 36 & 20 & 24.1 & 55.9 & 13.52 & 9 & 54.7 & 14.81 \\ 05: 28: 29: 35 & 20 & 24.1 & 55.9 & 14.27 & 9 & 54.8 & 14.81 \\ 05: 28: 30: 39 & 20 & 24.1 & 55.9 & 15.01 & 9 & 54.8 & 14.58 \\ 05: 28: 31: 38 & 20 & 24.1 & 55.8 & 15.81 & 11 & 54.8 & 14.58 \\ 05: 28: 32: 37 & 20 & 24.1 & 55.8 & 16.53 & 11 & 54.8 & 14.48 \\ 05: 28: 33: 36 & 20 & 24.1 & 55.8 & 17.30 & 9 & 54.8 & 14.48 \\ 05: 28: 34: 35 & 20 & 24.3 & 55.8 & 18.06 & 11 & 54.9 & 15.02 \\ 05: 28: 35: 39 & 20 & 24.1 & 55.8 & 18.79 & 9 & 54.9 & 15.02 \\ 05: 28: 36: 38 & 20 & 24.3 & 55.8 & 19.55 & 9 & 54.9 & 16.51\end{array}$


B10772.TXT

HNF-3557

Rev. 0

100IVD/8_IN_DRY_SAND_SN_B10772_750LB_55RPM_30SCFM_TO_COMPARE_WINO_FLOW_INSRT

TIME PURG_TC2 PurgePSI PurgeCFM DEPTH(IN) DwnFrc(lb) RPM Inches/Min

$\begin{array}{llllllll}06: 50: 20: 73 & 20 & -1.0 & 1.8 & 0.03 & -2 & 53.5 & -0.09 \\ 06: 50: 21: 72 & 20 & 14.6 & 57.3 & 0.31 & -1 & 53.5 & -0.49 \\ 06: 50: 22: 71 & 20 & 24.9 & 56.3 & 1.11 & 1 & 54.4 & -0.49 \\ 06: 50: 23: 70 & 20 & 24.3 & 56.2 & 1.89 & 1 & 54.4 & 10.64 \\ 06: 50: 24: 69 & 20 & 24.1 & 56.2 & 2.68 & 1 & 54.2 & 10.64 \\ 06: 50: 25: 68 & 20 & 23.9 & 56.2 & 3.46 & 1 & 54.2 & 14.71 \\ 06: 50: 26: 66 & 20 & 23.9 & 56.2 & 4.25 & 1 & 54.1 & 14.71 \\ 06: 50: 27: 65 & 20 & 24.1 & 56.2 & 5.00 & 1 & 54.1 & 14.62 \\ 06: 50: 28: 64 & 20 & 24.1 & 56.2 & 5.75 & 2 & 54.1 & 13.78 \\ 06: 50: 29: 63 & 20 & 24.1 & 56.2 & 6.53 & 1 & 54.1 & 13.78 \\ 06: 50: 30: 62 & 20 & 24.3 & 56.2 & 7.31 & 1 & 54.1 & 14.63 \\ 06: 50: 31: 61 & 20 & 24.3 & 56.2 & 8.07 & 2 & 54.1 & 14.63 \\ 06: 50: 32: 60 & 20 & 24.3 & 56.2 & 8.84 & 3 & 54.1 & 12.15 \\ 06: 50: 33: 58 & 20 & 24.3 & 56.2 & 9.64 & 2 & 54.2 & 12.15 \\ 06: 50: 34: 57 & 20 & 24.5 & 56.2 & 10.39 & 1 & 54.2 & 9.16 \\ 06: 50: 35: 56 & 20 & 24.3 & 56.2 & 11.13 & 14 & 54.3 & 9.16 \\ 06: 50: 36: 55 & 20 & 24.3 & 56.2 & 11.85 & 33 & 54.3 & 13.69 \\ 06: 50: 37: 54 & 20 & 24.5 & 56.1 & 12.61 & 2 & 54.3 & 13.69 \\ 06: 50: 38: 53 & 20 & 24.7 & 56.1 & 13.38 & 4 & 54.3 & 14.41 \\ 06: 50: 39: 52 & 20 & 24.7 & 56.1 & 14.17 & 3 & 54.3 & 14.41 \\ 06: 50: 40: 50 & 20 & 24.7 & 56.1 & 14.92 & 3 & 54.2 & 14.20 \\ 06: 50: 41: 49 & 20 & 24.7 & 56.1 & 15.70 & 3 & 54.2 & 14.20 \\ 06: 50: 42: 48 & 20 & 24.7 & 56.1 & 16.45 & 3 & 54.2 & 14.39 \\ 06: 50: 43: 47 & 20 & 24.7 & 56.1 & 17.19 & 2 & 54.2 & 14.39 \\ 06: 50: 44: 46 & 20 & 24.7 & 56.1 & 17.94 & 2 & 54.2 & 15.77 \\ 06: 50: 45: 45 & 20 & 24.7 & 56.1 & 18.68 & 2 & 54.1 & 15.77 \\ 06: 50: 46: 44 & 20 & 24.9 & 56.1 & 19.43 & 2 & 54.1 & 15.91\end{array}$


C10772.TXT

HNF-3557

Rev. 0

100IVD/8_IN_DRY_SAND_SN_C10772_750LB_55RPM_30SCFM_TO_COMPARE_WINO_FLOW_INSRT

TIME PURG_TC2 PurgePSI PurgeCFM DEPTH(IN) DwnFrc(lb) RPM Inches/Min

$\begin{array}{lccccccc}07: 07: 10: 81 & 20 & -0.8 & 1.8 & 0.02 & -2 & 55.5 & -0.40 \\ 07: 07: 11: 86 & 20 & 5.4 & 56.6 & 0.53 & -2 & 55.7 & 1.51 \\ 07: 07: 12: 90 & 20 & 21.4 & 55.9 & 1.35 & -2 & 55.7 & 1.51 \\ 07: 07: 13: 89 & 20 & 21.8 & 55.7 & 2.11 & -2 & 55.1 & 14.71 \\ 07: 07: 14: 88 & 20 & 21.6 & 55.6 & 2.90 & -2 & 55.1 & 14.71 \\ 07: 07: 15: 86 & 20 & 21.4 & 55.6 & 3.67 & -2 & 54.7 & 14.35 \\ 07: 07: 16: 85 & 20 & 21.6 & 55.5 & 4.43 & -1 & 54.7 & 14.35 \\ 07: 07: 17: 90 & 20 & 21.6 & 55.4 & 5.26 & -1 & 54.7 & 12.61 \\ 07: 07: 18: 89 & 20 & 21.6 & 55.1 & 6.03 & -1 & 54.7 & 12.61 \\ 07: 07: 19: 87 & 20 & 21.8 & 55.1 & 6.81 & 1 & 54.7 & 13.83 \\ 07: 07: 20: 92 & 20 & 21.8 & 54.9 & 7.61 & 1 & 54.8 & 13.83 \\ 07: 07: 21: 91 & 20 & 21.6 & 54.8 & 8.39 & 1 & 54.8 & 14.44 \\ 07: 07: 22: 90 & 20 & 21.8 & 54.7 & 9.15 & 1 & 54.8 & 14.44 \\ 07: 07: 23: 88 & 20 & 21.8 & 54.7 & 9.94 & 1 & 54.8 & 8.61 \\ 07: 07: 24: 87 & 20 & 21.8 & 54.6 & 10.69 & 1 & 54.8 & 8.61 \\ 07: 07: 25: 92 & 20 & 21.8 & 54.5 & 11.51 & 1 & 54.8 & 12.05 \\ 07: 07: 26: 90 & 20 & 21.8 & 54.5 & 12.29 & 1 & 54.8 & 14.45 \\ 07: 07: 27: 89 & 20 & 21.8 & 54.5 & 13.07 & 1 & 54.7 & 14.45 \\ 07: 07: 28: 88 & 20 & 21.8 & 54.5 & 13.87 & 1 & 54.7 & 14.70 \\ 07: 07: 29: 87 & 20 & 21.8 & 54.4 & 14.58 & 1 & 54.7 & 14.70 \\ 07: 07: 30: 91 & 20 & 21.8 & 54.3 & 15.40 & 1 & 54.7 & 14.56 \\ 07: 07: 31: 90 & 20 & 21.8 & 54.3 & 16.19 & -1 & 54.7 & 14.56 \\ 07: 07: 32: 89 & 20 & 21.8 & 54.2 & 16.92 & 1 & 54.7 & 14.49 \\ 07: 07: 33: 94 & 20 & 22.0 & 54.2 & 17.73 & 1 & 54.7 & 14.49 \\ 07: 07: 34: 92 & 20 & 21.8 & 54.2 & 18.48 & 1 & 54.8 & 16.46 \\ 07: 07: 35: 91 & 20 & 22.0 & 54.1 & 19.21 & 1 & 54.8 & 16.46\end{array}$


100IVD/8_IN_DRY_SAND_SN_D10772_750LB_55RPM_30SCFM_TO_COMPARE_WINO_FLOW_INSRT.

TIME PURG_TC2 PurgePSI PurgeCFM DEPTH(IN) DwnFrc(ib) RPM Inches/Min

$\begin{array}{llllllll}07: 51: 01: 47 & 21 & -0.6 & 1.7 & 0.09 & 2 & 55.6 & -0.10 \\ 07: 51: 02: 46 & 21 & 13.4 & 57.3 & 0.55 & 2 & 55.6 & -0.38 \\ 07: 51: 03: 45 & 21 & 22.9 & 55.8 & 1.32 & 3 & 55.6 & -0.38 \\ 07: 51: 04: 43 & 21 & 23.5 & 55.4 & 2.11 & 3 & 55.3 & 12.35 \\ 07: 51: 05: 42 & 21 & 23.3 & 54.7 & 2.87 & 2 & 54.6 & 12.35 \\ 07: 51: 06: 41 & 21 & 23.3 & 54.5 & 3.64 & 2 & 54.6 & 13.42 \\ 07: 51: 07: 40 & 21 & 23.3 & 54.4 & 4.42 & 3 & 54.5 & 13.42 \\ 07: 51: 08: 39 & 21 & 23.3 & 54.3 & 5.21 & 3 & 54.5 & 12.96 \\ 07: 51: 09: 38 & 21 & 23.3 & 54.2 & 5.96 & 3 & 54.5 & 13.43 \\ 07: 51: 10: 37 & 21 & 23.3 & 54.2 & 6.69 & 3 & 54.5 & 13.43 \\ 07: 51: 11: 35 & 21 & 23.3 & 54.1 & 7.48 & 3 & 54.4 & 14.60 \\ 07: 51: 12: 34 & 21 & 23.3 & 54.1 & 8.25 & 2 & 54.4 & 14.60 \\ 07: 51: 13: 33 & 21 & 23.3 & 54.1 & 9.04 & 3 & 54.4 & 10.16 \\ 07: 51: 14: 32 & 21 & 23.5 & 54.1 & 9.80 & 3 & 54.5 & 10.16 \\ 07: 51: 15: 31 & 21 & 23.3 & 54.1 & 10.57 & 4 & 54.5 & 9.64 \\ 07: 51: 16: 30 & 21 & 23.3 & 54.0 & 11.33 & 3 & 54.5 & 9.64 \\ 07: 51: 17: 29 & 21 & 23.3 & 54.0 & 12.10 & 4 & 54.5 & 13.73 \\ 07: 51: 18: 28 & 21 & 23.1 & 53.9 & 12.81 & 3 & 54.4 & 13.73 \\ 07: 51: 19: 26 & 21 & 23.3 & 53.9 & 13.64 & 3 & 54.4 & 13.98 \\ 07: 51: 20: 25 & 21 & 23.5 & 53.9 & 14.42 & 3 & 54.4 & 13.98 \\ 07: 51: 21: 24 & 21 & 23.3 & 53.9 & 15.18 & 3 & 54.3 & 14.74 \\ 07: 51: 22: 23 & 21 & 23.5 & 53.9 & 15.95 & 6 & 54.3 & 14.74 \\ 07: 51: 23: 22 & 21 & 23.5 & 53.8 & 16.70 & 6 & 54.3 & 14.13 \\ 07: 51: 24: 21 & 21 & 23.3 & 53.8 & 17.48 & 4 & 54.3 & 14.13 \\ 07: 51: 25: 20 & 21 & 23.3 & 53.8 & 18.23 & 3 & 54.3 & 15.42 \\ 07: 51: 26: 18 & 21 & 23.3 & 53.8 & 18.93 & 3 & 54.3 & 15.42\end{array}$


E10772.TXT

HNF-3557

Rev. 0

100IVD/8_IN_DRY_SAND_SN_E10772_750LB_55RPM_30SCFM_TO_COMPARE_WINO_FLOW_INSRT

TIME PURG_TC2 PurgePSI PurgeCFM DEPTH(IN) DwnFrc(lb) RPM Inches/Min

$\begin{array}{lllllllc}05: 19: 06: 09 & 19 & -0.6 & 1.7 & -0.05 & -9 & 55.8 & -0.26 \\ 05: 19: 07: 08 & 20 & 22.0 & 56.0 & 0.42 & -7 & 55.8 & -0.26 \\ 05: 19: 08: 12 & 20 & 24.3 & 54.1 & 1.23 & -9 & 55.8 & 9.76 \\ 05: 19: 09: 11 & 20 & 24.3 & 54.0 & 2.02 & -10 & 55.1 & 9.76 \\ 05: 19: 10: 10 & 19 & 24.1 & 53.8 & 2.76 & -10 & 54.6 & 13.40 \\ 05: 19: 11: 14 & 20 & 24.1 & 53.7 & 3.55 & -9 & 54.6 & 13.40 \\ 05: 19: 12: 13 & 19 & 24.3 & 53.6 & 4.33 & -9 & 54.5 & 13.25 \\ 05: 19: 13: 12 & 19 & 24.3 & 53.5 & 5.07 & -9 & 54.5 & 13.25 \\ 05: 19: 14: 11 & 20 & 24.3 & 53.5 & 5.86 & -10 & 54.5 & 13.00 \\ 05: 19: 15: 10 & 20 & 24.3 & 53.5 & 6.59 & -9 & 54.5 & 13.00 \\ 05: 19: 16: 14 & 20 & 24.3 & 53.5 & 7.37 & -9 & 54.6 & 13.74 \\ 05: 19: 17: 13 & 20 & 24.1 & 53.4 & 8.08 & -9 & 54.6 & 13.74 \\ 05: 19: 18: 12 & 19 & 24.1 & 53.4 & 8.83 & -9 & 54.6 & 11.19 \\ 05: 19: 19: 16 & 20 & 24.1 & 53.4 & 9.64 & -9 & 54.5 & 11.19 \\ 05: 19: 20: 15 & 19 & 23.9 & 53.4 & 10.40 & -10 & 54.5 & 8.43 \\ 05: 19: 21: 14 & 19 & 24.1 & 53.4 & 11.12 & -10 & 54.5 & 8.43 \\ 05: 19: 22: 13 & 19 & 24.1 & 53.4 & 11.91 & -9 & 54.5 & 12.76 \\ 05: 19: 23: 12 & 19 & 23.9 & 53.3 & 12.61 & -9 & 54.5 & 12.76 \\ 05: 19: 24: 16 & 19 & 23.9 & 53.3 & 13.42 & -9 & 54.5 & 14.05 \\ 05: 19: 25: 15 & 20 & 23.9 & 53.3 & 14.19 & -9 & 54.6 & 14.05 \\ 05: 19: 26: 14 & 19 & 23.9 & 53.3 & 14.90 & -10 & 54.6 & 13.88 \\ 05: 19: 27: 18 & 20 & 23.9 & 53.3 & 15.68 & -9 & 54.6 & 13.66 \\ 05: 19: 28: 17 & 19 & 23.9 & 53.3 & 16.46 & -10 & 54.5 & 13.66 \\ 05: 19: 29: 16 & 19 & 23.9 & 53.3 & 17.16 & -9 & 54.5 & 13.80 \\ 05: 19: 30: 15 & 19 & 23.9 & 53.3 & 17.95 & -10 & 54.5 & 13.80 \\ 05: 19: 31: 13 & 19 & 23.9 & 53.3 & 18.65 & -9 & 54.5 & 14.98 \\ 05: 19: 32: 18 & 19 & 24.1 & 53.3 & 19.38 & -8 & 54.5 & 14.98\end{array}$


F10772.TXT

HNF-3557

Rev. 0

100IVD/8_IN_DRY_UREA_SN_F10772_750LB_55RPM_3OSCFM_TO_COMPARE_WINO_FLOW_INSRT

TIME PURG_TC2 PurgePSI PurgeCFM DEPTH(IN) DwnFrc(lb) RPM Inches/Min

$\begin{array}{llllllll}05: 40: 35: 08 & 19 & -0.6 & 1.8 & -0.04 & 1 & 56.1 & -0.45 \\ 05: 40: 36: 07 & 20 & -0.4 & 58.7 & 0.25 & 1 & 56.1 & -0.40 \\ 05: 40: 37: 06 & 20 & 23.7 & 54.2 & 1.03 & 0 & 56.1 & -0.40 \\ 05: 40: 38: 05 & 20 & 24.1 & 54.0 & 1.78 & 0 & 55.6 & 13.73 \\ 05: 40: 39: 03 & 20 & 24.1 & 53.6 & 2.56 & 4 & 55.1 & 13.73 \\ 05: 40: 40: 02 & 20 & 24.3 & 53.5 & 3.34 & 6 & 55.1 & 13.68 \\ 05: 40: 41: 01 & 20 & 24.5 & 53.5 & 4.06 & 8 & 55.0 & 13.68 \\ 05: 40: 42: 00 & 20 & 24.5 & 53.4 & 4.84 & 9 & 55.0 & 13.18 \\ 05: 40: 42: 99 & 20 & 24.7 & 53.3 & 5.63 & 11 & 55.0 & 13.18 \\ 05: 40: 43: 98 & 20 & 24.7 & 53.2 & 6.36 & 14 & 55.0 & 13.49 \\ 05: 40: 44: 97 & 20 & 24.7 & 53.2 & 7.11 & 18 & 55.0 & 13.49 \\ 05: 40: 45: 95 & 20 & 24.7 & 53.0 & 7.88 & 21 & 55.1 & 13.95 \\ 05: 40: 46: 94 & 20 & 24.7 & 52.9 & 8.60 & 24 & 55.1 & 13.95 \\ 05: 40: 47: 93 & 19 & 24.9 & 52.9 & 9.37 & 31 & 55.1 & 9.87 \\ 05: 40: 48: 92 & 19 & 24.9 & 52.9 & 10.10 & 36 & 55.1 & 9.87 \\ 05: 40: 49: 91 & 20 & 24.9 & 52.8 & 10.78 & 39 & 55.1 & 9.11 \\ 05: 40: 50: 90 & 20 & 24.9 & 52.8 & 11.51 & 40 & 55.1 & 9.11 \\ 05: 40: 51: 94 & 20 & 24.9 & 52.8 & 12.26 & 44 & 55.1 & 13.31 \\ 05: 40: 52: 93 & 20 & 24.9 & 52.7 & 13.00 & 49 & 55.0 & 13.31 \\ 05: 40: 53: 92 & 20 & 24.9 & 52.7 & 13.73 & 55 & 55.0 & 13.67 \\ 05: 40: 54: 91 & 20 & 24.9 & 52.7 & 14.42 & 64 & 55.0 & 13.67 \\ 05: 40: 55: 90 & 20 & 24.9 & 52.7 & 15.12 & 70 & 55.0 & 13.65 \\ 05: 40: 56: 89 & 20 & 24.9 & 52.6 & 15.78 & 75 & 55.0 & 12.86 \\ 05: 40: 57: 87 & 20 & 24.9 & 52.6 & 16.44 & 80 & 55.0 & 12.86 \\ 05: 40: 58: 86 & 20 & 24.9 & 52.6 & 17.09 & 94 & 54.9 & 14.16 \\ 05: 40: 59: 85 & 20 & 25.1 & 52.6 & 17.76 & 100 & 54.9 & 14.16 \\ 05: 41: 00: 84 & 20 & 25.1 & 52.6 & 18.38 & 104 & 54.8 & 15.49 \\ 05: 41: 01: 83 & 20 & 25.1 & 52.6 & 18.98 & 110 & 54.8 & 15.49\end{array}$


100IVD/8_IN_DRY_UREA_SN_G10772_750LB_55RPM_30SCFM_TO_COMPARE_W/NO_FLOW_INSRT

TIME PURG_TC2 PurgePSI PurgeCFM DEPTH(IN) DwnFrc(Ib) RPM Inches/Min

$\begin{array}{lllllllc}05: 53: 33: 87 & 20 & -0.6 & 1.8 & 0.10 & -1 & 53.9 & -0.46 \\ 05: 53: 34: 86 & 20 & 21.8 & 24.0 & 0.48 & -2 & 54.9 & -0.46 \\ 05: 53: 35: 85 & 20 & 23.3 & 24.0 & 1.25 & -1 & 54.9 & 5.05 \\ 05: 53: 36: 89 & 20 & 23.5 & 24.0 & 2.02 & 1 & 55.0 & 5.05 \\ 05: 53: 37: 88 & 20 & 23.5 & 23.9 & 2.84 & 1 & 54.6 & 13.10 \\ 05: 53: 38: 87 & 20 & 23.7 & 23.9 & 3.62 & 5 & 54.6 & 13.10 \\ 05: 53: 39: 91 & 20 & 23.9 & 23.8 & 4.41 & 8 & 54.5 & 13.49 \\ 05: 53: 40: 90 & 20 & 23.9 & 23.8 & 5.21 & 9 & 54.6 & 13.49 \\ 05: 53: 41: 89 & 20 & 23.9 & 23.8 & 5.96 & 10 & 54.6 & 12.75 \\ 05: 53: 42: 88 & 20 & 24.1 & 23.8 & 6.75 & 14 & 54.5 & 13.55 \\ 05: 53: 43: 86 & 20 & 24.1 & 23.8 & 7.51 & 18 & 54.6 & 13.55 \\ 05: 53: 44: 91 & 20 & 24.3 & 23.6 & 8.28 & 21 & 54.6 & 10.81 \\ 05: 53: 45: 90 & 20 & 24.3 & 23.6 & 9.13 & 25 & 54.7 & 10.81 \\ 05: 53: 46: 89 & 20 & 24.3 & 23.5 & 9.84 & 30 & 54.6 & 8.67 \\ 05: 53: 47: 87 & 20 & 24.3 & 23.5 & 10.63 & 36 & 54.6 & 8.67 \\ 05: 53: 48: 86 & 20 & 24.3 & 23.3 & 11.37 & 37 & 54.6 & 12.55 \\ 05: 53: 49: 91 & 20 & 24.3 & 23.3 & 12.10 & 44 & 54.6 & 12.55 \\ 05: 53: 50: 90 & 20 & 24.3 & 23.0 & 12.85 & 46 & 54.6 & 13.69 \\ 05: 53: 51: 88 & 20 & 24.3 & 22.9 & 13.59 & 56 & 54.5 & 13.69 \\ 05: 53: 52: 87 & 20 & 24.3 & 22.8 & 14.30 & 60 & 54.5 & 13.63 \\ 05: 53: 53: 86 & 20 & 24.3 & 22.8 & 15.00 & 72 & 54.5 & 13.63 \\ 05: 53: 54: 90 & 20 & 24.3 & 22.7 & 15.71 & 82 & 54.5 & 13.21 \\ 05: 53: 55: 89 & 20 & 24.5 & 22.6 & 16.42 & 91 & 54.5 & 13.21 \\ 05: 53: 56: 88 & 20 & 24.3 & 22.5 & 17.07 & 109 & 54.5 & 13.61 \\ 05: 53: 57: 93 & 20 & 24.3 & 22.4 & 17.71 & 124 & 54.6 & 13.61 \\ 05: 53: 58: 91 & 20 & 24.7 & 22.3 & 18.31 & 141 & 54.6 & 14.49 \\ 05: 53: 59: 90 & 20 & 24.5 & 22.1 & 18.88 & 150 & 54.6 & 14.49\end{array}$


100IVD/8_IN_DRY_UREA_SN_H10772_750LB_55RPM_30SCFM_TO_COMPARE_WINO_FLOW_INSRT

TIME PURG_TC2 PurgePSI PurgeCFM DEPTH(IN) DwnFrc(Ib) RPM Inches/Min

$\begin{array}{lllllllc}06: 18: 54: 81 & 20 & -0.6 & 1.7 & 0.05 & -1 & 55.7 & -0.04 \\ 06: 18: 55: 80 & 20 & 15.4 & 70.0 & 0.55 & -2 & 55.7 & 3.83 \\ 06: 18: 56: 79 & 20 & 22.9 & 70.0 & 1.35 & -1 & 55.7 & 3.83 \\ 06: 18: 57: 78 & 20 & 22.9 & 70.0 & 2.14 & 0 & 55.3 & 13.28 \\ 06: 18: 58: 76 & 20 & 22.9 & 70.0 & 2.90 & 3 & 54.8 & 13.28 \\ 06: 18: 59: 75 & 20 & 23.1 & 70.0 & 3.68 & 5 & 54.8 & 13.85 \\ 06: 19: 00: 74 & 20 & 23.1 & 70.0 & 4.46 & 8 & 54.7 & 13.85 \\ 06: 19: 01: 73 & 20 & 23.5 & 70.0 & 5.22 & 10 & 54.6 & 13.39 \\ 06: 19: 02: 72 & 20 & 23.5 & 70.0 & 6.02 & 14 & 54.6 & 13.39 \\ 06: 19: 03: 71 & 20 & 23.5 & 70.0 & 6.75 & 15 & 54.7 & 13.25 \\ 06: 19: 04: 70 & 20 & 23.5 & 70.0 & 7.53 & 19 & 54.8 & 13.25 \\ 06: 19: 05: 69 & 20 & 23.5 & 70.0 & 8.30 & 23 & 54.8 & 12.61 \\ 06: 19: 06: 67 & 20 & 23.5 & 70.0 & 9.06 & 26 & 54.8 & 12.61 \\ 06: 19: 07: 66 & 20 & 23.5 & 70.0 & 9.83 & 34 & 54.8 & 8.53 \\ 06: 19: 08: 65 & 20 & 23.7 & 70.0 & 10.55 & 39 & 54.8 & 8.53 \\ 06: 19: 09: 64 & 20 & 23.5 & 70.0 & 11.28 & 44 & 54.8 & 11.34 \\ 06: 19: 10: 63 & 20 & 23.5 & 70.0 & 12.02 & 46 & 54.8 & 11.34 \\ 06: 19: 11: 62 & 20 & 23.7 & 70.0 & 12.73 & 55 & 54.8 & 13.17 \\ 06: 19: 12: 61 & 20 & 23.7 & 70.0 & 13.44 & 65 & 54.8 & 13.17 \\ 06: 19: 13: 59 & 20 & 23.7 & 70.0 & 14.16 & 71 & 54.8 & 13.49 \\ 06: 19: 14: 58 & 20 & 23.7 & 70.0 & 14.83 & 84 & 54.8 & 13.49 \\ 06: 19: 15: 57 & 20 & 23.7 & 70.0 & 15.49 & 100 & 54.9 & 13.58 \\ 06: 19: 16: 56 & 20 & 23.7 & 70.0 & 16.13 & 117 & 54.9 & 13.58 \\ 06: 19: 17: 55 & 20 & 23.7 & 70.0 & 16.75 & 129 & 54.9 & 13.56 \\ 06: 19: 18: 54 & 20 & 23.7 & 70.0 & 17.39 & 135 & 54.9 & 13.56 \\ 06: 19: 19: 53 & 20 & 23.9 & 70.0 & 17.99 & 148 & 54.8 & 13.52 \\ 06: 19: 20: 52 & 20 & 23.7 & 70.0 & 18.55 & 157 & 54.8 & 13.52 \\ 06: 19: 21: 50 & 20 & 23.9 & 70.0 & 19.07 & 161 & 54.8 & 15.25\end{array}$


100IVD/8_IN_DRY_KMAG_SN_I10772_750LB_55RPM_30SCFM_TO_COMPARE_WINO_FLOW_INSRT

TIME PURG_TC2 PurgePSI PurgeCFM DEPTH(IN) DwnFrc(lb) RPM Inches/Min

$\begin{array}{lccccccc}08: 02: 13: 65 & 20 & -0.6 & 70.0 & 0.04 & -1 & 55.6 & -0.52 \\ 08: 02: 14: 58 & 20 & 9.1 & 70.0 & 0.49 & -1 & 55.6 & 3.45 \\ 08: 02: 15: 57 & 20 & 23.1 & 70.0 & 1.25 & 0 & 55.6 & 3.45 \\ 08: 02: 16: 56 & 20 & 22.9 & 53.9 & 2.04 & 0 & 55.1 & 13.38 \\ 08: 02: 17: 55 & 20 & 23.1 & 53.9 & 2.84 & 5 & 54.5 & 13.38 \\ 08: 02: 18: 53 & 20 & 23.1 & 53.9 & 3.61 & 15 & 54.5 & 13.83 \\ 08: 02: 19: 52 & 20 & 23.3 & 53.9 & 4.34 & 19 & 54.4 & 13.83 \\ 08: 02: 20: 51 & 20 & 23.7 & 53.9 & 5.12 & 24 & 54.4 & 13.12 \\ 08: 02: 21: 50 & 20 & 23.9 & 53.9 & 5.89 & 28 & 54.4 & 13.12 \\ 08: 02: 22: 49 & 20 & 23.7 & 53.9 & 6.61 & 32 & 54.5 & 13.68 \\ 08: 02: 23: 48 & 20 & 23.9 & 53.9 & 7.37 & 41 & 54.5 & 13.68 \\ 08: 02: 24: 47 & 20 & 23.9 & 53.8 & 8.11 & 44 & 54.5 & 13.23 \\ 08: 02: 25: 46 & 20 & 24.1 & 53.8 & 8.83 & 49 & 54.4 & 13.23 \\ 08: 02: 26: 44 & 20 & 24.1 & 53.8 & 9.56 & 58 & 54.3 & 8.58 \\ 08: 02: 27: 43 & 20 & 24.3 & 53.8 & 10.29 & 66 & 54.3 & 8.58 \\ 08: 02: 28: 42 & 20 & 24.3 & 53.8 & 11.01 & 84 & 54.2 & 9.45 \\ 08: 02: 29: 41 & 20 & 24.3 & 53.7 & 11.68 & 80 & 54.2 & 9.45 \\ 08: 02: 30: 40 & 20 & 24.3 & 53.7 & 12.36 & 86 & 54.2 & 13.58 \\ 08: 02: 31: 39 & 20 & 24.1 & 53.7 & 13.05 & 89 & 54.1 & 13.58 \\ 08: 02: 32: 38 & 20 & 24.3 & 53.6 & 13.74 & 90 & 54.1 & 13.90 \\ 08: 02: 33: 36 & 20 & 24.3 & 53.6 & 14.40 & 96 & 54.1 & 13.90 \\ 08: 02: 34: 35 & 20 & 24.1 & 53.5 & 15.07 & 100 & 54.1 & 13.37 \\ 08: 02: 35: 34 & 20 & 24.1 & 53.5 & 15.74 & 106 & 54.1 & 13.37 \\ 08: 02: 36: 33 & 20 & 24.1 & 53.4 & 16.36 & 111 & 54.1 & 14.06 \\ 08: 02: 37: 32 & 20 & 24.3 & 53.4 & 16.99 & 116 & 54.1 & 14.06 \\ 08: 02: 38: 31 & 20 & 24.3 & 53.4 & 17.63 & 125 & 54.2 & 14.55 \\ 08: 02: 39: 30 & 20 & 24.3 & 53.4 & 18.22 & 128 & 54.2 & 14.55 \\ 08: 02: 40: 29 & 20 & 24.3 & 53.4 & 18.78 & 132 & 54.2 & 14.08 \\ 08: 02: 41: 27 & 20 & 24.5 & 53.4 & 19.33 & 140 & 54.2 & 14.08\end{array}$


100IVD18_IN_DRY_KMAG_SN_J10772_750LB_55RPM_30SCFM_TO_COMPARE_WINO_FLOW_INSRT

TIME PURG_TC2 PurgePSI PurgeCFM DEPTH(IN) DwnFrc(lb) RPM Inches/Min

$\begin{array}{lllllllc}08: 17: 46: 89 & 20 & -0.6 & 1.7 & 0.04 & 3 & 55.6 & -0.38 \\ 08: 17: 47: 87 & 20 & 22.8 & 55.6 & 0.29 & 0 & 55.6 & -0.38 \\ 08: 17: 48: 86 & 20 & 24.1 & 54.3 & 1.06 & 12 & 55.7 & 7.23 \\ 08: 17: 49: 85 & 20 & 24.9 & 53.8 & 1.82 & 28 & 55.0 & 7.23 \\ 08: 17: 50: 84 & 20 & 24.9 & 53.4 & 2.60 & 42 & 55.0 & 13.22 \\ 08: 17: 51: 83 & 20 & 24.9 & 53.3 & 3.31 & 49 & 54.8 & 13.22 \\ 08: 17: 52: 82 & 20 & 25.1 & 53.1 & 4.06 & 61 & 54.7 & 13.41 \\ 08: 17: 53: 81 & 20 & 25.3 & 53.0 & 4.75 & 76 & 54.7 & 13.41 \\ 08: 17: 54: 79 & 20 & 25.1 & 52.9 & 5.48 & 87 & 54.8 & 11.63 \\ 08: 17: 55: 78 & 20 & 25.3 & 52.8 & 6.17 & 100 & 54.7 & 11.63 \\ 08: 17: 56: 77 & 20 & 25.3 & 52.8 & 6.83 & 110 & 54.7 & 12.99 \\ 08: 17: 57: 76 & 20 & 25.3 & 52.7 & 7.51 & 119 & 54.7 & 12.99 \\ 08: 17: 58: 75 & 20 & 25.5 & 52.6 & 8.14 & 125 & 54.8 & 12.70 \\ 08: 17: 59: 74 & 20 & 25.5 & 52.6 & 8.78 & 134 & 54.8 & 12.70 \\ 08: 18: 00: 73 & 20 & 25.7 & 52.6 & 9.42 & 137 & 54.8 & 7.34 \\ 08: 18: 01: 72 & 20 & 25.5 & 52.5 & 10.06 & 133 & 54.8 & 7.34 \\ 08: 18: 02: 70 & 20 & 25.7 & 52.5 & 10.67 & 143 & 54.8 & 9.65 \\ 08: 18: 03: 69 & 20 & 25.9 & 52.5 & 11.28 & 144 & 54.8 & 9.65 \\ 08: 18: 04: 68 & 20 & 25.9 & 52.4 & 11.88 & 148 & 54.7 & 13.19 \\ 08: 18: 05: 67 & 20 & 25.7 & 52.4 & 12.49 & 150 & 54.7 & 13.19 \\ 08: 18: 06: 66 & 20 & 25.7 & 52.3 & 13.14 & 152 & 54.7 & 12.39 \\ 08: 18: 07: 65 & 20 & 25.5 & 52.3 & 13.74 & 152 & 54.7 & 12.39 \\ 08: 18: 08: 64 & 20 & 25.3 & 52.3 & 14.30 & 163 & 54.7 & 13.14 \\ 08: 18: 09: 62 & 20 & 25.5 & 52.3 & 14.89 & 173 & 54.7 & 13.14 \\ 08: 18: 10: 61 & 20 & 25.5 & 52.2 & 15.46 & 173 & 54.7 & 12.74 \\ 08: 18: 11: 60 & 20 & 25.7 & 52.2 & 16.02 & 200 & 54.7 & 12.74 \\ 08: 18: 12: 59 & 20 & 25.7 & 52.2 & 16.55 & 203 & 54.7 & 12.23 \\ 08: 18: 13: 58 & 20 & 25.7 & 52.2 & 17.08 & 208 & 54.7 & 12.23 \\ 08: 18: 14: 57 & 20 & 25.7 & 52.2 & 17.63 & 215 & 54.6 & 13.71 \\ 08: 18: 15: 56 & 20 & 25.9 & 52.2 & 18.13 & 226 & 54.6 & 13.71 \\ 08: 18: 16: 55 & 20 & 26.1 & 52.2 & 18.56 & 238 & 54.6 & 13.23 \\ 08: 18: 17: 53 & 20 & 26.1 & 52.2 & 18.97 & 293 & 54.6 & 12.61\end{array}$


100IVD/8_IN_DRY_KMAG_SN_K10772_750LB_55RPM_30SCFM_TO_COMPARE_WINO_FLOW_INSRT

TIME PURG_TC2 PurgePSI PurgeCFM DEPTH(IN) DwnFrc(lb) RPM Inches/Min

$\begin{array}{lccccccc}08: 30: 17: 11 & 20 & -0.6 & 1.7 & 0.04 & 2.0 & 53.5 & -0.25 \\ 08: 30: 18: 10 & 20 & 5.6 & 56.2 & 0.29 & 3.3 & 53.5 & -0.50 \\ 08: 30: 19: 09 & 20 & 23.5 & 54.6 & 1.11 & 17.0 & 54.4 & -0.50 \\ 08: 30: 20: 08 & 20 & 23.5 & 54.2 & 1.82 & 28.3 & 54.3 & 10.30 \\ 08: 30: 21: 12 & 20 & 23.5 & 54.1 & 2.61 & 34.5 & 54.3 & 10.30 \\ 08: 30: 22: 11 & 20 & 23.9 & 54.0 & 3.38 & 39.5 & 54.3 & 12.40 \\ 08: 30: 23: 10 & 20 & 23.7 & 53.8 & 4.09 & 52.0 & 54.3 & 12.40 \\ 08: 30: 24: 09 & 20 & 23.9 & 53.6 & 4.84 & 78.3 & 54.3 & 13.05 \\ 08: 30: 25: 08 & 20 & 24.1 & 53.5 & 5.51 & 97.0 & 54.2 & 13.05 \\ 08: 30: 26: 12 & 20 & 24.3 & 53.5 & 6.21 & 120.8 & 54.2 & 12.12 \\ 08: 30: 27: 11 & 20 & 24.3 & 53.4 & 6.88 & 135.8 & 54.2 & 12.49 \\ 08: 30: 28: 10 & 20 & 24.5 & 53.3 & 7.47 & 144.5 & 54.2 & 12.49 \\ 08: 30: 29: 09 & 20 & 24.5 & 53.3 & 8.11 & 144.5 & 54.2 & 11.77 \\ 08: 30: 30: 08 & 20 & 24.5 & 53.1 & 8.74 & 147.0 & 54.2 & 11.77 \\ 08: 30: 31: 12 & 20 & 24.7 & 52.9 & 9.37 & 152.0 & 54.2 & 7.21 \\ 08: 30: 32: 11 & 20 & 24.7 & 52.8 & 9.95 & 155.8 & 54.0 & 7.21 \\ 08: 30: 33: 10 & 20 & 24.9 & 52.8 & 10.52 & 165.8 & 54.0 & 9.35 \\ 08: 30: 34: 14 & 20 & 25.1 & 52.8 & 11.12 & 174.5 & 53.9 & 9.35 \\ 08: 30: 35: 13 & 20 & 25.1 & 52.7 & 11.70 & 174.5 & 53.9 & 12.00 \\ 08: 30: 36: 28 & 20 & 25.1 & 52.7 & 12.22 & 182.0 & 53.9 & 12.00 \\ 08: 30: 37: 11 & 20 & 25.1 & 52.7 & 12.79 & 192.0 & 53.9 & 12.97 \\ 08: 30: 38: 09 & 20 & 24.9 & 52.7 & 13.36 & 197.0 & 53.8 & 12.97 \\ 08: 30: 39: 08 & 20 & 25.1 & 52.6 & 13.92 & 200.8 & 53.8 & 12.83 \\ 08: 30: 40: 07 & 20 & 25.1 & 52.6 & 14.43 & 212.0 & 53.7 & 12.83 \\ 08: 30: 41: 06 & 20 & 25.1 & 52.6 & 14.93 & 218.3 & 53.6 & 13.00 \\ 08: 30: 42: 05 & 20 & 25.1 & 52.6 & 15.42 & 228.3 & 53.6 & 13.00 \\ 08: 30: 43: 04 & 20 & 25.3 & 52.6 & 15.92 & 238.3 & 53.5 & 11.80 \\ 08: 30: 44: 03 & 20 & 25.3 & 52.6 & 16.39 & 249.5 & 53.5 & 11.80 \\ 08: 30: 45: 01 & 20 & 25.5 & 52.6 & 16.90 & 249.5 & 53.5 & 13.23 \\ 08: 30: 46: 00 & 20 & 25.3 & 52.6 & 17.38 & 254.5 & 53.3 & 13.23 \\ 08: 30: 46: 99 & 20 & 25.5 & 52.6 & 17.85 & 284.5 & 53.4 & 13.40 \\ 08: 30: 47: 98 & 20 & 25.5 & 52.6 & 18.20 & 347.0 & 53.4 & 13.40 \\ 08: 30: 48: 97 & 20 & 25.5 & 52.6 & 18.45 & 448.3 & 53.4 & 11.27 \\ 08: 30: 49: 96 & 20 & 25.5 & 52.6 & 18.65 & 422.0 & 53.5 & 11.27 \\ 08: 30: 50: 95 & 20 & 25.7 & 52.6 & 18.84 & 489.5 & 53.5 & 7.79 \\ 08: 30: 51: 94 & 20 & 25.7 & 52.6 & 19.02 & 530.8 & 53.8 & 7.79\end{array}$


100IVD/8_IN_DRY_KMAG_SN_L10772_750LB_55RPM_3OSCFM_TO_COMPARE_WINO_FLOW_INSRT TIME PURG_TC2 PurgePSI PurgeCFM DEPTH(IN) DwnFrc(Ib) RPM Inches/Min

$\begin{array}{llllllll}08: 53: 49: 74 & 20 & -0.6 & 1.7 & -0.03 & 0 & 55.4 & -0.59 \\ 08: 53: 50: 79 & 20 & 23.3 & 70.0 & 0.58 & 6 & 55.6 & -0.59 \\ 08: 53: 51: 83 & 20 & 24.5 & 70.0 & 1.39 & 15 & 55.4 & 10.55 \\ 08: 53: 52: 82 & 20 & 24.9 & 70.0 & 2.16 & 24 & 55.4 & 10.55 \\ 08: 53: 53: 81 & 20 & 25.1 & 70.0 & 2.92 & 33 & 54.8 & 12.49 \\ 08: 53: 54: 79 & 20 & 25.1 & 70.0 & 3.68 & 43 & 54.7 & 12.49 \\ 08: 53: 55: 78 & 20 & 25.1 & 70.0 & 4.42 & 49 & 54.7 & 12.92 \\ 08: 53: 56: 83 & 20 & 25.5 & 70.0 & 5.19 & 58 & 54.6 & 12.92 \\ 08: 53: 57: 82 & 20 & 25.5 & 70.0 & 5.95 & 67 & 54.6 & 11.72 \\ 08: 53: 58: 80 & 20 & 25.5 & 70.0 & 6.64 & 75 & 54.6 & 11.72 \\ 08: 53: 59: 85 & 20 & 25.7 & 70.0 & 7.39 & 91 & 54.6 & 13.01 \\ 08: 54: 00: 84 & 20 & 25.5 & 70.0 & 8.07 & 101 & 54.7 & 13.01 \\ 08: 54: 01: 83 & 20 & 25.7 & 70.0 & 8.74 & 112 & 54.7 & 10.95 \\ 08: 54: 02: 81 & 20 & 25.9 & 70.0 & 9.41 & 123 & 54.7 & 10.95 \\ 08: 54: 03: 80 & 20 & 25.7 & 70.0 & 10.03 & 127 & 54.8 & 7.86 \\ 08: 54: 04: 85 & 20 & 25.7 & 70.0 & 10.68 & 133 & 54.8 & 7.86 \\ 08: 54: 05: 83 & 20 & 25.7 & 70.0 & 11.28 & 153 & 54.7 & 11.01 \\ 08: 54: 06: 82 & 20 & 25.7 & 70.0 & 11.85 & 158 & 54.7 & 11.01 \\ 08: 54: 07: 87 & 20 & 25.9 & 70.0 & 12.44 & 170 & 54.7 & 11.60 \\ 08: 54: 08: 86 & 20 & 25.9 & 70.0 & 13.03 & 180 & 54.8 & 12.23 \\ 08: 54: 09: 84 & 20 & 25.9 & 70.0 & 13.58 & 200 & 54.8 & 12.23 \\ 08: 54: 10: 83 & 20 & 26.3 & 70.0 & 14.12 & 214 & 54.8 & 11.48 \\ 08: 54: 11: 82 & 20 & 26.1 & 70.0 & 14.61 & 237 & 54.8 & 11.48 \\ 08: 54: 12: 87 & 20 & 26.3 & 70.0 & 15.11 & 243 & 54.7 & 12.05 \\ 08: 54: 13: 85 & 20 & 26.3 & 70.0 & 15.55 & 266 & 54.7 & 12.05 \\ 08: 54: 14: 84 & 20 & 26.1 & 70.0 & 16.02 & 275 & 54.7 & 10.81 \\ 08: 54: 15: 83 & 20 & 26.3 & 70.0 & 16.46 & 277 & 54.7 & 10.81 \\ 08: 54: 16: 82 & 20 & 26.5 & 70.0 & 16.85 & 281 & 54.7 & 11.73 \\ 08: 54: 17: 86 & 20 & 26.6 & 70.0 & 17.31 & 290 & 54.6 & 11.73 \\ 08: 54: 18: 85 & 20 & 26.8 & 70.0 & 17.71 & 348 & 54.5 & 11.89 \\ 08: 54: 19: 84 & 20 & 26.8 & 70.0 & 17.95 & 451 & 54.5 & 11.89 \\ 08: 54: 20: 88 & 20 & 27.0 & 70.0 & 18.17 & 455 & 54.5 & 7.91 \\ 08: 54: 21: 87 & 20 & 26.8 & 70.0 & 18.33 & 498 & 54.7 & 7.91 \\ 08: 54: 22: 86 & 20 & 27.0 & 70.0 & 18.48 & 535 & 54.7 & 6.31 \\ 08: 54: 23: 85 & 20 & 26.8 & 70.0 & 18.62 & 537 & 54.7 & 6.31\end{array}$


TIME PURG_TC2 PurgePSI PurgeCFM DEPTH(IN) DWnFrc(Ib) RPM Inches/Min

$\begin{array}{llllllll}08: 54: 24: 84 & 20 & 27.0 & 70.0 & 18.89 & 363 & 54.5 & 6.04 \\ 08: 54: 25: 88 & 20 & 27.0 & 70.0 & 19.06 & 469 & 54.5 & 6.04\end{array}$


100IVD/9_IN_DRY_KMAG_SN_A-NF1_750LB_55RPM_30SCFM_TO_COMPARE_W/3PIN_INSERT TIME PURG_TC2 PurgePSI PurgeCFM DEPTH(IN) DwnFrc(Ib) RPM Inches/Min

$\begin{array}{lllllllc}09: 32: 31: 72 & 20 & -0.6 & 9.0 & -0.03 & 1 & 55.7 & -0.14 \\ 09: 32: 32: 71 & 20 & 20.6 & 55.5 & 0.66 & 5 & 55.6 & 4.06 \\ 09: 32: 33: 69 & 20 & 21.4 & 55.5 & 1.44 & 15 & 55.1 & 4.06 \\ 09: 32: 34: 68 & 20 & 21.6 & 55.4 & 2.20 & 17 & 55.1 & 13.08 \\ 09: 32: 35: 67 & 21 & 21.4 & 55.4 & 3.01 & 16 & 54.6 & 13.08 \\ 09: 32: 36: 66 & 20 & 21.6 & 55.2 & 3.77 & 24 & 54.6 & 12.86 \\ 09: 32: 37: 65 & 20 & 21.8 & 54.7 & 4.55 & 35 & 54.6 & 12.86 \\ 09: 32: 38: 64 & 20 & 21.8 & 54.5 & 5.28 & 50 & 54.6 & 13.41 \\ 09: 32: 39: 63 & 21 & 22.2 & 54.2 & 5.99 & 64 & 54.6 & 13.41 \\ 09: 32: 40: 61 & 20 & 22.6 & 54.0 & 6.75 & 81 & 54.5 & 12.32 \\ 09: 32: 41: 60 & 20 & 22.8 & 53.8 & 7.43 & 86 & 54.5 & 12.32 \\ 09: 32: 42: 59 & 20 & 22.8 & 53.7 & 8.14 & 98 & 54.5 & 12.97 \\ 09: 32: 43: 58 & 20 & 22.8 & 53.6 & 8.83 & 103 & 54.3 & 12.97 \\ 09: 32: 44: 57 & 20 & 22.9 & 53.5 & 9.48 & 118 & 54.1 & 7.92 \\ 09: 32: 45: 56 & 20 & 23.3 & 53.4 & 10.12 & 131 & 54.1 & 7.92 \\ 09: 32: 46: 55 & 20 & 23.7 & 53.3 & 10.73 & 144 & 53.9 & 9.97 \\ 09: 32: 47: 54 & 20 & 24.3 & 52.9 & 11.33 & 156 & 53.8 & 9.97 \\ 09: 32: 48: 52 & 20 & 24.1 & 52.8 & 11.94 & 161 & 53.8 & 12.36 \\ 09: 32: 49: 51 & 20 & 23.7 & 52.8 & 12.53 & 164 & 53.9 & 12.36 \\ 09: 32: 50: 50 & 20 & 24.5 & 52.7 & 13.13 & 176 & 53.9 & 13.58 \\ 09: 32: 51: 49 & 20 & 23.7 & 52.7 & 13.69 & 188 & 53.9 & 13.58 \\ 09: 32: 52: 48 & 20 & 23.9 & 52.6 & 14.24 & 202 & 53.7 & 12.72 \\ 09: 32: 53: 47 & 20 & 24.1 & 52.6 & 14.77 & 210 & 53.6 & 12.72 \\ 09: 32: 54: 46 & 20 & 25.3 & 52.5 & 15.30 & 220 & 53.6 & 12.91 \\ 09: 32: 55: 44 & 20 & 25.9 & 52.1 & 15.79 & 231 & 53.6 & 12.91 \\ 09: 32: 56: 43 & 20 & 25.9 & 52.0 & 16.27 & 241 & 53.6 & 12.80 \\ 09: 32: 57: 42 & 20 & 26.1 & 51.8 & 16.73 & 253 & 53.6 & 12.80 \\ 09: 32: 58: 41 & 20 & 26.6 & 51.7 & 17.15 & 351 & 53.6 & 12.65 \\ 09: 32: 59: 40 & 20 & 26.6 & 51.4 & 17.41 & 371 & 53.6 & 12.65 \\ 09: 33: 00: 39 & 20 & 26.8 & 51.2 & 17.71 & 376 & 53.6 & 10.55 \\ 09: 33: 01: 38 & 20 & 26.8 & 51.1 & 17.97 & 428 & 53.8 & 10.55 \\ 09: 33: 02: 37 & 20 & 26.8 & 51.0 & 18.20 & 473 & 54.1 & 8.98 \\ 09: 33: 03: 35 & 20 & 27.2 & 50.9 & 18.36 & 493 & 54.1 & 8.98 \\ 09: 33: 04: 34 & 20 & 27.0 & 50.8 & 18.55 & 425 & 54.1 & 6.97 \\ 09: 33: 05: 33 & 20 & 27.0 & 50.7 & 18.69 & 539 & 54.2 & 5.98 \\ 09: 33: 06: 32 & 20 & 27.0 & 50.7 & 18.92 & 495 & 54.2 & 5.98 \\ 09: 33: 07: 31 & 20 & 27.0 & 50.7 & 19.07 & 521 & 54.2 & 6.79\end{array}$


100IVD/9_IN_DRY_KMAG_SN_B-NFI_750LB_55RPM_30SCFM_TO_COMPARE_W/3PIN_INSERT TIME PURG_TC2 PurgePSI PurgeCFM DEPTH(IN) DwnFrc(lb) RPM Inches/Min

$\begin{array}{llllllll}09: 45: 48: 03 & 20 & -0.6 & 1.7 & -0.02 & 3 & 55.7 & -0.42 \\ 09: 45: 49: 02 & 21 & 21.2 & 56.5 & 0.56 & 3 & 55.9 & -0.42 \\ 09: 45: 50: 00 & 21 & 21.6 & 56.1 & 1.36 & 7 & 55.9 & 7.19 \\ 09: 45: 50: 99 & 21 & 21.4 & 55.9 & 2.09 & 14 & 55.3 & 7.19 \\ 09: 45: 51: 98 & 21 & 21.8 & 55.8 & 2.92 & 14 & 54.8 & 13.06 \\ 09: 45: 52: 97 & 21 & 21.8 & 55.7 & 3.68 & 18 & 54.8 & 13.06 \\ 09: 45: 53: 96 & 21 & 22.0 & 55.6 & 4.46 & 25 & 54.8 & 13.61 \\ 09: 45: 54: 95 & 20 & 22.0 & 55.6 & 5.22 & 35 & 54.7 & 13.61 \\ 09: 45: 55: 94 & 21 & 22.2 & 55.4 & 6.00 & 45 & 54.7 & 13.00 \\ 09: 45: 56: 92 & 20 & 22.6 & 54.7 & 6.72 & 64 & 54.8 & 13.00 \\ 09: 45: 57: 91 & 21 & 22.9 & 54.3 & 7.42 & 75 & 54.8 & 12.59 \\ 09: 45: 58: 90 & 21 & 23.1 & 54.1 & 8.14 & 87 & 54.8 & 12.59 \\ 09: 45: 59: 89 & 21 & 23.5 & 53.9 & 8.83 & 93 & 54.9 & 11.60 \\ 09: 46: 00: 88 & 20 & 23.9 & 53.7 & 9.50 & 105 & 54.8 & 11.60 \\ 09: 46: 01: 87 & 20 & 24.1 & 53.5 & 10.17 & 110 & 54.8 & 8.05 \\ 09: 46: 02: 86 & 20 & 24.9 & 53.2 & 10.82 & 125 & 54.8 & 8.05 \\ 09: 46: 03: 85 & 21 & 25.3 & 52.8 & 11.45 & 141 & 54.8 & 10.10 \\ 09: 46: 04: 83 & 21 & 25.7 & 52.7 & 12.11 & 147 & 54.8 & 12.44 \\ 09: 46: 05: 82 & 21 & 26.1 & 52.5 & 12.70 & 159 & 54.7 & 12.44 \\ 09: 46: 06: 81 & 20 & 24.3 & 52.2 & 13.30 & 173 & 54.7 & 13.48 \\ 09: 46: 07: 80 & 21 & 23.9 & 52.2 & 13.91 & 174 & 54.7 & 13.48 \\ 09: 46: 08: 79 & 20 & 24.1 & 52.2 & 14.43 & 194 & 54.7 & 12.04 \\ 09: 46: 09: 78 & 20 & 24.3 & 52.2 & 14.95 & 205 & 54.6 & .12 .04 \\ 09: 46: 10: 77 & 20 & 24.5 & 52.2 & 15.46 & 222 & 54.6 & 12.73 \\ 09: 46: 11: 75 & 20 & 24.7 & 52.2 & 15.97 & 238 & 54.5 & 12.73 \\ 09: 46: 12: 74 & 20 & 25.1 & 52.2 & 16.44 & 251 & 54.5 & 12.43 \\ 09: 46: 13: 73 & 20 & 25.1 & 52.2 & 16.93 & 257 & 54.5 & 12.43 \\ 09: 46: 14: 72 & 20 & 25.1 & 52.2 & 17.39 & 260 & 54.4 & 13.08 \\ 09: 46: 15: 71 & 21 & 25.3 & 52.2 & 17.81 & 276 & 54.4 & 13.08 \\ 09: 46: 16: 70 & 20 & 25.3 & 52.2 & 18.23 & 290 & 54.4 & 12.26 \\ 09: 46: 17: 69 & 20 & 25.1 & 52.1 & 18.59 & 298 & 54.4 & 12.26 \\ 09: 46: 18: 67 & 20 & 25.1 & 52.1 & 18.97 & 305 & 54.3 & 11.82\end{array}$


100IVD/9_IN_DRY_KMAG_SN_C-NFI_750LB_55RPM_30SCFM_TO_COMPARE_W/3PIN_INSERT TIM E PURG_TC2 PurgePSI PurgeCFM DEPTH(IN) DwnFrc(lb) RPM Inches/Min

$\begin{array}{llllllll}09: 58: 27: 70 & 21 & -0.6 & 1.7 & 0.02 & 2 & 56.0 & 0.05 \\ 09: 58: 28: 69 & 21 & 18.5 & 70.0 & 0.33 & 1 & 56.0 & -0.24 \\ 09: 58: 29: 68 & 21 & 20.8 & 70.0 & 1.08 & 4 & 56.0 & -0.24 \\ 09: 58: 30: 72 & 21 & 21.2 & 70.0 & 1.90 & 11 & 55.3 & 9.37 \\ 09: 58: 31: 71 & 21 & 21.2 & 70.0 & 2.71 & 11 & 55.3 & 12.86 \\ 09: 58: 32: 70 & 21 & 21.2 & 70.0 & 3.47 & 12 & 55.0 & 12.86 \\ 09: 58: 33: 74 & 21 & 21.4 & 70.0 & 4.28 & 14 & 54.9 & 12.65 \\ 09: 58: 34: 73 & 21 & 21.6 & 70.0 & 5.11 & 27 & 54.9 & 12.65 \\ 09: 58: 35: 72 & 21 & 21.6 & 70.0 & 5.82 & 33 & 54.8 & 12.73 \\ 09: 58: 36: 71 & 21 & 21.8 & 70.0 & 6.59 & 60 & 54.6 & 12.73 \\ 09: 58: 37: 70 & 21 & 21.8 & 69.9 & 7.24 & 110 & 54.6 & 12.57 \\ 09: 58: 38: 74 & 21 & 22.4 & 70.0 & 7.94 & 121 & 54.6 & 12.57 \\ 09: 58: 39: 73 & 21 & 22.8 & 70.0 & 8.62 & 123 & 54.5 & 10.31 \\ 09: 58: 40: 72 & 21 & 23.5 & 70.0 & 9.27 & 125 & 54.5 & 10.31 \\ 09: 58: 41: 76 & 21 & 23.5 & 70.0 & 9.93 & 129 & 54.5 & 7.83 \\ 09: 58: 42: 75 & 21 & 23.9 & 70.0 & 10.59 & 127 & 54.5 & 7.83 \\ 09: 58: 43: 74 & 21 & 24.1 & 70.0 & 11.23 & 133 & 54.5 & 11.26 \\ 09: 58: 44: 73 & 21 & 25.1 & 70.0 & 11.87 & 143 & 54.5 & 11.26 \\ 09: 58: 45: 72 & 21 & 24.3 & 70.0 & 12.42 & 158 & 54.4 & 13.63 \\ 09: 58: 46: 76 & 20 & 24.7 & 70.0 & 13.08 & 174 & 54.4 & 13.63 \\ 09: 58: 47: 75 & 21 & 25.9 & 70.0 & 13.67 & 185 & 54.4 & 12.27 \\ 09: 58: 48: 74 & 21 & 26.1 & 70.0 & 14.16 & 208 & 54.4 & 12.27 \\ 09: 58: 49: 78 & 21 & 25.5 & 70.0 & 14.70 & 208 & 54.4 & 12.71 \\ 09: 58: 50: 77 & 21 & 26.3 & 70.0 & 15.25 & 212 & 54.4 & 12.71 \\ 09: 58: 51: 76 & 21 & 26.5 & 70.0 & 15.72 & 231 & 54.3 & 12.71 \\ 09: 58: 52: 75 & 21 & 27.2 & 70.0 & 16.23 & 248 & 54.3 & 11.75 \\ 09: 58: 53: 74 & 21 & 28.0 & 70.0 & 16.66 & 250 & 54.3 & 11.75 \\ 09: 58: 54: 78 & 21 & 28.4 & 70.0 & 17.15 & 262 & 54.3 & 12.69 \\ 09: 58: 55: 77 & 21 & 28.2 & 70.0 & 17.59 & 275 & 54.3 & 12.69 \\ 09: 58: 56: 76 & 21 & 28.4 & 70.0 & 17.95 & 278 & 54.3 & 12.04 \\ 09: 58: 57: 75 & 21 & 28.8 & 70.0 & 18.34 & 292 & 54.3 & 12.04 \\ 09: 58: 58: 73 & 21 & 29.0 & 70.0 & 18.66 & 309 & 54.3 & 11.10 \\ 09: 58: 59: 72 & 21 & 29.0 & 70.0 & 19.02 & 302 & 54.2 & 11.10\end{array}$


100IVD/9_IN_DRY_UREA_SN_D-NFI_750LB_55RPM_30SCFM_TO_COMPARE_W/3PIN_INSERT TIME PURG_TC2 PurgePSI PurgeCFM DEPTH(IN) DwnFrc(lb) RPM Inches/Min

\begin{tabular}{|c|c|c|c|c|c|c|c|}
\hline $10: 07: 48: 05$ & 21 & -0.6 & 70.0 & 0.02 & -2 & 55.6 & -0.10 \\
\hline $10: 07: 49: 04$ & 21 & 20.0 & 70.0 & 0.34 & -2 & 55.6 & -0.10 \\
\hline $10: 07: 50: 03$ & 21 & 21.2 & 70.0 & 1.11 & -1 & 55.7 & 8.55 \\
\hline $10: 07: 51: 02$ & 21 & 21.2 & 70.0 & 1.92 & -0 & 55.1 & 8.55 \\
\hline $10: 07: 52: 01$ & 21 & 21.2 & 70.0 & 2.72 & 2 & 55.1 & 13.09 \\
\hline $10: 07: 52: 99$ & 21 & 21.4 & 70.0 & 3.51 & 5 & 54.8 & 13.09 \\
\hline $10: 07: 54: 04$ & 21 & 21.6 & 70.0 & 4.35 & 8 & 54.8 & 12.89 \\
\hline $10: 07: 55: 03$ & 21 & 21.6 & 70.0 & 5.15 & 12 & 54.8 & 12.89 \\
\hline $10: 07: 56: 02$ & 21 & 21.6 & 70.0 & 5.95 & 16 & 54.8 & 13.46 \\
\hline $10: 07: 57: 00$ & 21 & 21.6 & 70.0 & 6.72 & 17 & 54.8 & 13.46 \\
\hline 10:07:57:99 & 21 & 21.8 & 70.0 & 7.50 & 22 & 54.8 & 12.67 \\
\hline $10: 07: 59: 04$ & 21 & 22.0 & 70.0 & 8.32 & 27 & 54.8 & 12.67 \\
\hline 10:08:00:02 & 21 & 22.2 & 70.0 & 9.09 & 34 & 54.8 & 8.62 \\
\hline 10:08:01:01 & 21 & 22.4 & 70.0 & 9.82 & 40 & 54.8 & 8.62 \\
\hline 10:08:02:06 & 21 & 22.6 & 70.0 & 10.61 & 58 & 54.8 & 9.89 \\
\hline 10:08:03:05 & 21 & 22.9 & 70.0 & 11.34 & 59 & 54.8 & 9.89 \\
\hline $10: 08: 04: 03$ & 21 & 23.3 & 70.0 & 12.05 & 67 & 54.8 & 12.82 \\
\hline $10: 08: 05: 02$ & 21 & 23.9 & 70.0 & 12.79 & 74 & 54.8 & 12.82 \\
\hline 10:08:06:01 & 21 & 23.9 & 70.0 & 13.48 & 82 & 54.7 & 12.92 \\
\hline 10:08:07:06 & 21 & 24.5 & 70.0 & 14.18 & 99 & 54.7 & 12.92 \\
\hline 10:08:08:04 & 21 & 24.5 & 70.0 & 14.83 & 110 & 54.7 & 13.07 \\
\hline $10: 08: 09: 03$ & 21 & 25.3 & 70.0 & 15.48 & 124 & 54.5 & 13.07 \\
\hline $10: 08: 10: 08$ & 21 & 25.9 & 70.0 & 16.13 & 143 & 54.5 & 13.22 \\
\hline $10: 08: 11: 06$ & 21 & 26.3 & 70.0 & 16.74 & 144 & 54.5 & 14.93 \\
\hline $10: 08: 12: 05$ & 21 & 26.3 & 70.0 & 17.31 & 159 & 54.5 & 14.93 \\
\hline $10: 08: 13: 04$ & 21 & 26.8 & 70.0 & 17.84 & 171 & 54.5 & 14.17 \\
\hline $10: 08: 14: 03$ & 21 & 27.4 & 70.0 & 18.33 & 190 & 54.4 & 14.17 \\
\hline $10: 08: 15: 07$ & 21 & 27.2 & 70.0 & 18.64 & 367 & 54.5 & 11.22 \\
\hline 10:08:16:06 & 21 & 27.2 & 70.0 & 19.05 & 265 & 54.5 & 11.22 \\
\hline 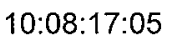 & 21 & 3 & 70.0 & 19.27 & 470 & 54.7 & 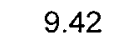 \\
\hline
\end{tabular}


100IVD/9_IN_DRY_UREA_SN_E-NFI_750LB_55RPM_30SCFM_TO_COMPARE_W/3PIN_INSERT TIME PURG_TC2 PurgePSI PurgeCFM DEPTH(IN) DwnFrc(lb) RPM Inches/Min

$\begin{array}{lllllllc}10: 17: 40: 75 & 21 & -0.8 & 70.0 & 0.05 & -6 & 56.1 & 0.14 \\ 10: 17: 41: 74 & 21 & 20.0 & 70.0 & 0.51 & -8 & 56.1 & 0.14 \\ 10: 17: 42: 73 & 21 & 21.0 & 70.0 & 1.29 & -8 & 55.7 & 5.98 \\ 10: 17: 43: 72 & 21 & 21.0 & 70.0 & 2.09 & -6 & 55.7 & 5.98 \\ 10: 17: 44: 71 & 21 & 21.4 & 70.0 & 2.90 & -5 & 55.2 & 12.67 \\ 10: 17: 45: 70 & 21 & 21.2 & 70.0 & 3.68 & -3 & 55.2 & 12.67 \\ 10: 17: 46: 68 & 21 & 21.2 & 70.0 & 4.49 & -0 & 55.2 & 12.95 \\ 10: 17: 47: 67 & 21 & 21.4 & 70.0 & 5.28 & 3 & 55.2 & 12.95 \\ 10: 17: 48: 66 & 21 & 21.4 & 70.0 & 6.06 & 5 & 55.2 & 12.78 \\ 10: 17: 49: 65 & 21 & 21.4 & 70.0 & 6.86 & 6 & 55.1 & 12.78 \\ 10: 17: 50: 64 & 21 & 21.6 & 70.0 & 7.63 & 12 & 55.0 & 13.68 \\ 10: 17: 51: 63 & 21 & 21.8 & 70.0 & 8.42 & 18 & 55.0 & 13.68 \\ 10: 17: 52: 62 & 21 & 22.0 & 70.0 & 9.18 & 24 & 55.0 & 8.41 \\ 10: 17: 53: 60 & 21 & 22.0 & 70.0 & 9.95 & 36 & 54.9 & 8.41 \\ 10: 17: 54: 59 & 21 & 22.4 & 70.0 & 10.69 & 41 & 54.9 & 10.66 \\ 10: 17: 55: 58 & 21 & 22.8 & 70.0 & 11.42 & 41 & 54.9 & 13.59 \\ 10: 17: 56: 57 & 21 & 22.9 & 70.0 & 12.17 & 45 & 54.8 & 13.59 \\ 10: 17: 57: 56 & 21 & 23.1 & 70.0 & 12.88 & 61 & 54.8 & 12.79 \\ 10: 17: 58: 55 & 21 & 23.7 & 70.0 & 13.62 & 71 & 54.8 & 12.79 \\ 10: 17: 59: 54 & 21 & 24.3 & 70.0 & 14.28 & 81 & 54.7 & 13.37 \\ 10: 18: 00: 53 & 21 & 24.7 & 70.0 & 14.97 & 89 & 54.7 & 13.37 \\ 10: 18: 01: 51 & 21 & 25.1 & 70.0 & 15.61 & 100 & 54.8 & 12.21 \\ 10: 18: 02: 50 & 21 & 25.9 & 70.0 & 16.23 & 114 & 54.8 & 12.21 \\ 10: 18: 03: 49 & 21 & 26.3 & 70.0 & 16.86 & 129 & 54.8 & 13.67 \\ 10: 18: 04: 48 & 21 & 26.5 & 70.0 & 17.43 & 162 & 54.7 & 13.67 \\ 10: 18: 05: 47 & 21 & 26.8 & 70.0 & 17.97 & 171 & 54.7 & 14.57 \\ 10: 18: 06: 46 & 21 & 27.4 & 70.0 & 18.52 & 164 & 54.7 & 14.57 \\ 10: 18: 07: 45 & 21 & 28.0 & 70.0 & 19.01 & 183 & 54.6 & 13.80\end{array}$




\begin{tabular}{|c|c|c|c|c|c|c|c|}
\hline TIME & PURG_TC2 & PurgePSI & PurgeCFM & DEPTH(IN) & DwnFrc(lb) & ) RPM & lnches/Min \\
\hline $10: 48: 42: 95$ & 20 & -0.6 & 1.7 & -0.01 & -8 & 48.4 & -0.42 \\
\hline $10: 48: 43: 93$ & 21 & 14.2 & 54.0 & 0.54 & -7 & 48.4 & -0.42 \\
\hline $10: 48: 44: 92$ & 21 & 21.0 & 54.0 & 1.33 & -6 & 50.3 & 7.27 \\
\hline $10: 48: 45: 91$ & 21 & 21.2 & 54.0 & 2.13 & -5 & 51.2 & 7.27 \\
\hline $10: 48: 46: 90$ & 21 & 21.2 & 54.0 & 2.92 & -3 & 51.2 & 12.50 \\
\hline $10: 48: 47: 89$ & 21 & 21.2 & 54.0 & 3.70 & 1 & 52.3 & 12.50 \\
\hline $10: 48: 48: 88$ & 21 & 21.4 & 54.0 & 4.52 & 4 & 53.2 & 13.18 \\
\hline $10: 48: 49: 87$ & 21 & 21.4 & 54.0 & 5.27 & 4 & 53.2 & 13.18 \\
\hline $10: 48: 50: 86$ & 21 & 21.4 & 54.0 & 6.07 & 8 & 53.7 & 13.44 \\
\hline $10: 48: 51: 84$ & 21 & 21.6 & 54.0 & 6.86 & 12 & 54.2 & 13.44 \\
\hline $10: 48: 52: 83$ & 21 & 21.6 & 54.0 & 7.63 & 15 & 54.2 & 12.90 \\
\hline $10: 48: 53: 82$ & 21 & 21.8 & 54.0 & 8.38 & 20 & 54.5 & 12.90 \\
\hline $10: 48: 54: 81$ & 21 & 21.8 & 53.9 & 9.16 & 27 & 54.6 & 9.00 \\
\hline $10: 48: 55: 80$ & 20 & 22.4 & 53.8 & 9.8 & 32 & 54.6 & 9.00 \\
\hline $10: 48: 56: 79$ & 20 & 22.4 & 53.7 & 10.66 & 39 & 54.6 & 7.89 \\
\hline $10: 48: 57: 78$ & 21 & 22.6 & 53.6 & 11.39 & 45 & 54.6 & 7.89 \\
\hline $10: 48: 5$ & 21 & 22.9 & 53.5 & 12.12 & 54 & 54.6 & 12.28 \\
\hline $10: 48: 59: 75$ & 21 & 23.1 & 53.4 & 12.86 & 69 & 54.5 & 13.48 \\
\hline $10: 49: 00: 74$ & 21 & 23.5 & 53.2 & 13.57 & 73 & 54.5 & 13.48 \\
\hline $10: 49: 01: 73$ & 21 & 24.1 & 52.8 & 14.25 & 81 & 54.5 & 13.32 \\
\hline $10: 49: 02: 72$ & 21 & 24.5 & 52.6 & 14.92 & 94 & 54.5 & 13.32 \\
\hline $10: 49: 03: 71$ & 21 & 25.3 & 52.2 & 15.55 & 116 & 54.5 & 13.90 \\
\hline $10: 49: 04: 70$ & 21 & 25.9 & 51.4 & 16.17 & 131 & 54.5 & 13.90 \\
\hline $10: 49: 0$ & 21 & 26.5 & 51.0 & 16.78 & 145 & 54.6 & 15.10 \\
\hline $10: 49: 06: 67$ & 21 & 26.6 & 50.6 & 17.36 & 157 & 54.6 & 15.10 \\
\hline $10: 49: 07: 66$ & 21 & 27.6 & 50.4 & 17.89 & 166 & 54.6 & 14.06 \\
\hline $10: 49: 08: 65$ & 21 & 28.2 & 50.0 & 18.44 & 177 & 54.6 & 14.06 \\
\hline & 21 & 28.4 & 49.8 & 18.96 & 159 & 54.6 & 13.93 \\
\hline $10: 49: 10: 63$ & 21 & 28.2 & 49.7 & 19.46 & 191 & 54.6 & 13.93 \\
\hline
\end{tabular}


100IVD/9_IN_DRY_SAND_SN_G-NFI_750LB_55RPM_30SCFM_TO_COMPARE_W/3PIN_INSERT TIME PURG_TC2 PurgePSI PurgeCFM DEPTH(IN) DwnFrc(lb) RPM Inches/Min

$\begin{array}{llllllll}10: 58: 03: 79 & 21 & -0.6 & 1.7 & -0.01 & 1 & 55.8 & -1.28 \\ 10: 58: 04: 72 & 21 & 14.6 & 24.3 & 0.11 & 1 & 55.8 & -1.28 \\ 10: 58: 05: 71 & 21 & 20.8 & 24.3 & 0.91 & -0 & 55.8 & 3.38 \\ 10: 58: 06: 70 & 21 & 20.8 & 24.2 & 1.71 & -0 & 55.2 & 3.38 \\ 10: 58: 07: 69 & 21 & 21.2 & 24.2 & 2.54 & 1 & 55.2 & 13.48 \\ 10: 58: 08: 68 & 21 & 21.0 & 24.2 & 3.31 & 2 & 54.9 & 13.48 \\ 10: 58: 09: 72 & 21 & 21.0 & 24.2 & 4.16 & 2 & 54.9 & 13.12 \\ 10: 58: 10: 71 & 21 & 21.0 & 24.2 & 5.01 & 2 & 54.9 & 13.12 \\ 10: 58: 11: 70 & 21 & 21.0 & 24.2 & 5.79 & 2 & 54.8 & 13.10 \\ 10: 58: 12: 74 & 21 & 21.2 & 24.2 & 6.62 & 2 & 54.8 & 13.10 \\ 10: 58: 13: 73 & 21 & 21.2 & 24.2 & 7.46 & 2 & 54.8 & 13.71 \\ 10: 58: 14: 72 & 21 & 21.2 & 24: 2 & 8.24 & 2 & 54.8 & 10.07 \\ 10: 58: 15: 71 & 20 & 21.0 & 24.1 & 9.08 & 2 & 54.7 & 10.07 \\ 10: 58: 16: 70 & 21 & 21.2 & 24.0 & 9.82 & 3 & 54.7 & 9.17 \\ 10: 58: 17: 74 & 21 & 21.0 & 23.9 & 10.66 & 1 & 54.7 & 9.17 \\ 10: 58: 18: 73 & 21 & 21.2 & 23.9 & 11.49 & 2 & 54.8 & 11.88 \\ 10: 58: 19: 72 & 21 & 21.2 & 23.9 & 12.26 & 1 & 54.8 & 11.88 \\ 10: 58: 20: 76 & 21 & 21.2 & 23.8 & 13.11 & 2 & 54.8 & 14.72 \\ 10: 58: 21: 75 & 21 & 21.2 & 23.7 & 13.94 & 3 & 54.8 & 14.72 \\ 10: 58: 22: 74 & 21 & 21.0 & 23.6 & 14.68 & 2 & 54.8 & 13.15 \\ 10: 58: 23: 73 & 21 & 21.2 & 23.6 & 15.52 & 2 & 54.8 & 13.15 \\ 10: 58: 24: 72 & 21 & 21.0 & 23.6 & 16.26 & 2 & 54.7 & 13.62 \\ 10: 58: 25: 76 & 21 & 21.2 & 23.5 & 17.10 & 2 & 54.7 & 13.62 \\ 10: 58: 26: 75 & 21 & 21.2 & 23.5 & 17.88 & 3 & 54.7 & 14.69 \\ 10: 58: 27: 74 & 21 & 21.2 & 23.5 & 18.45 & -0 & 54.7 & 14.69 \\ 10: 58: 28: 78 & 21 & 21.2 & 23.4 & 19.09 & 1 & 54.7 & 12.05\end{array}$


100IVD/9_IN_DRY_SAND_SN_H-NFI_750LB_55RPM_30SCFM_TO_COMPARE_W/3PIN_INSERT

TIME PURG_TC2 PurgePSI PurgeCFM DEPTH(IN) DwnFrc(Ib) RPM Inches/Min

$\begin{array}{llllllll}11: 07: 12: 17 & 21 & -0.6 & 1.7 & -0.03 & 4 & 55.6 & -0.05 \\ 11: 07: 13: 10 & 21 & 12.8 & 14.4 & 0.31 & 5 & 55.9 & -0.88 \\ 11: 07: 14: 09 & 21 & 21.2 & 14.4 & 1.10 & 3 & 55.7 & -0.88 \\ 11: 07: 15: 08 & 21 & 21.2 & 14.4 & 1.90 & 4 & 55.7 & 9.94 \\ 11: 07: 16: 07 & 21 & 21.2 & 14.4 & 2.69 & 3 & 55.1 & 12.50 \\ 11: 07: 17: 05 & 21 & 21.0 & 14.4 & 3.50 & 3 & 55.0 & 12.50 \\ 11: 07: 18: 04 & 21 & 21.0 & 14.4 & 4.31 & 3 & 55.0 & 12.08 \\ 11: 07: 19: 03 & 21 & 21.0 & 14.4 & 5.10 & 4 & 55.1 & 12.08 \\ 11: 07: 20: 02 & 21 & 21.2 & 14.4 & 5.89 & 4 & 55.1 & 12.80 \\ 11: 07: 21: 01 & 21 & 21.2 & 14.5 & 6.70 & 5 & 55.1 & 12.80 \\ 11: 07: 22: 00 & 21 & 21.2 & 14.5 & 7.49 & 7 & 55.1 & 12.69 \\ 11: 07: 22: 99 & 21 & 21.2 & 14.5 & 8.30 & 4 & 55.1 & 12.69 \\ 11: 07: 23: 98 & 21 & 21.2 & 14.6 & 9.09 & 5 & 55.0 & 9.32 \\ 11: 07: 24: 96 & 21 & 21.2 & 14.6 & 9.89 & 5 & 55.0 & 9.32 \\ 11: 07: 25: 95 & 21 & 21.4 & 14.6 & 10.68 & 5 & 55.0 & 8.72 \\ 11: 07: 26: 94 & 21 & 21.2 & 14.7 & 11.47 & 4 & 54.9 & 8.72 \\ 11: 07: 27: 93 & 21 & 21.2 & 14.7 & 12.28 & 4 & 54.8 & 13.78 \\ 11: 07: 28: 92 & 21 & 21.2 & 14.7 & 13.09 & 5 & 54.8 & 13.78 \\ 11: 07: 29: 91 & 21 & 21.2 & 14.7 & 13.85 & 4 & 54.7 & 13.74 \\ 11: 07: 30: 90 & 21 & 21.2 & 14.7 & 14.67 & 5 & 54.6 & 13.74 \\ 11: 07: 31: 88 & 21 & 21.2 & 14.7 & 15.47 & 5 & 54.6 & 14.12 \\ 11: 07: 32: 87 & 21 & 21.2 & 14.7 & 16.25 & 6 & 54.6 & 14.12 \\ 11: 07: 33: 86 & 21 & 21.2 & 14.7 & 17.04 & 4 & 54.6 & 14.25 \\ 11: 07: 34: 85 & 20 & 21.2 & 14.7 & 17.80 & 3 & 54.6 & 14.25 \\ 11: 07: 35: 84 & 21 & 21.2 & 14.7 & 18.57 & 3 & 54.6 & 15.42 \\ 11: 07: 36: 83 & 21 & 21.2 & 14.7 & 19.33 & 3 & 54.7 & 15.42\end{array}$


100IVD/9_IN_DRY_SAND_SN_I-NFI_750LB_55RPM_30SCFM_TO_COMPARE_W/3PIN_INSERT

TIME PURG_TC2 PurgePSI PurgeCFM DEPTH(IN) DwnFrc(lb) RPM Inches/Min

$\begin{array}{llllllll}11: 16: 21: 59 & 21 & -0.6 & 1.7 & 0.05 & 2 & 54.3 & -0.59 \\ 11: 16: 22: 57 & 21 & 20.2 & 23.0 & 0.59 & 1 & 54.3 & -0.59 \\ 11: 16: 23: 62 & 21 & 21.2 & 23.0 & 1.42 & 2 & 54.8 & 8.80 \\ 11: 16: 24: 61 & 21 & 21.2 & 22.9 & 2.26 & 1 & 54.6 & 8.80 \\ 11: 16: 25: 60 & 21 & 21.2 & 22.9 & 3.04 & 2 & 54.6 & 12.87 \\ 11: 16: 26: 58 & 21 & 21.2 & 22.9 & 3.87 & 4 & 54.6 & 12.87 \\ 11: 16: 27: 57 & 21 & 21.2 & 22.9 & 4.64 & 4 & 54.7 & 12.92 \\ 11: 16: 28: 62 & 21 & 21.2 & 22.8 & 5.51 & 4 & 54.7 & 12.92 \\ 11: 16: 29: 60 & 21 & 21.2 & 22.8 & 6.34 & 7 & 54.7 & 13.45 \\ 11: 16: 30: 59 & 21 & 21.2 & 22.7 & 7.08 & 5 & 54.8 & 13.45 \\ 11: 16: 31: 64 & 21 & 21.4 & 22.6 & 7.92 & 4 & 54.8 & 12.58 \\ 11: 16: 32: 63 & 21 & 21.2 & 22.6 & 8.78 & 2 & 54.8 & 12.58 \\ 11: 16: 33: 61 & 21 & 21.2 & 22.6 & 9.52 & 2 & 54.8 & 9.17 \\ 11: 16: 34: 60 & 21 & 21.4 & 22.6 & 10.34 & 4 & 54.8 & 9.17 \\ 11: 16: 35: 59 & 21 & 21.2 & 22.5 & 11.11 & 4 & 54.8 & 10.03 \\ 11: 16: 36: 64 & 21 & 21.2 & 22.4 & 11.94 & 4 & 54.8 & 10.03 \\ 11: 16: 37: 62 & 21 & 21.4 & 22.4 & 12.79 & 6 & 54.8 & 13.65 \\ 11: 16: 38: 61 & 21 & 21.4 & 22.3 & 13.53 & 3 & 54.8 & 13.65 \\ 11: 16: 39: 66 & 21 & 21.4 & 22.2 & 14.37 & 3 & 54.7 & 13.67 \\ 11: 16: 40: 64 & 21 & 21.4 & 22.2 & 15.20 & 4 & 54.7 & 13.33 \\ 11: 16: 41: 63 & 21 & 21.4 & 22.2 & 15.93 & 2 & 54.8 & 13.33 \\ 11: 16: 42: 62 & 21 & 21.4 & 22.1 & 16.78 & 4 & 54.7 & 15.38 \\ 11: 16: 43: 61 & 21 & 21.4 & 22.1 & 17.53 & 6 & 54.7 & 15.38 \\ 11: 16: 44: 65 & 21 & 21.4 & 22.0 & 18.31 & 6 & 54.6 & 14.40 \\ 11: 16: 45: 64 & 21 & 21.4 & 22.0 & 19.12 & 6 & 54.6 & 14.40\end{array}$


100IVD/8_IN_UREA_SN_M10772_750LB_55RPM_30SCFM_TO_COMPARE_NOFLOW_INSERT

TIME PURG_TC2 PurgePSI PurgeCFM DEPTH(IN) DwnFrc(lb) RPM Inches/Min

$\begin{array}{llllllll}11: 26: 57: 51 & 21 & -0.6 & 1.7 & 0.03 & 3 & 55.7 & -0.03 \\ 11: 26: 58: 50 & 21 & 14.6 & 70.0 & 0.46 & 2 & 55.7 & -0.35 \\ 11: 26: 59: 49 & 21 & 23.7 & 70.0 & 1.25 & 0 & 55.7 & -0.35 \\ 11: 27: 00: 48 & 21 & 23.7 & 70.0 & 2.06 & 1 & 55.1 & 11.93 \\ 11: 27: 01: 47 & 21 & 24.1 & 70.0 & 2.85 & 5 & 55.1 & 11.93 \\ 11: 27: 02: 46 & 21 & 23.9 & 70.0 & 3.62 & 7 & 54.9 & 13.45 \\ 11: 27: 03: 45 & 21 & 24.1 & 70.0 & 4.43 & 10 & 54.7 & 13.45 \\ 11: 27: 04: 43 & 21 & 24.3 & 70.0 & 5.22 & 13 & 54.7 & 12.53 \\ 11: 27: 05: 42 & 21 & 24.5 & 70.0 & 5.98 & 16 & 54.7 & 12.53 \\ 11: 27: 06: 41 & 21 & 24.5 & 70.0 & 6.76 & 19 & 54.8 & 13.11 \\ 11: 27: 07: 40 & 21 & 24.5 & 70.0 & 7.54 & 23 & 54.8 & 13.11 \\ 11: 27: 08: 39 & 21 & 24.5 & 70.0 & 8.32 & 27 & 54.9 & 13.27 \\ 11: 27: 09: 38 & 21 & 24.7 & 70.0 & 9.07 & 35 & 55.0 & 8.93 \\ 11: 27: 10: 37 & 21 & 24.7 & 70.0 & 9.80 & 39 & 55.0 & 8.93 \\ 11: 27: 11: 35 & 21 & 24.7 & 70.0 & 10.54 & 48 & 55.1 & 7.80 \\ 11: 27: 12: 34 & 21 & 24.7 & 70.0 & 11.26 & 59 & 55.0 & 7.80 \\ 11: 27: 13: 33 & 21 & 24.9 & 70.0 & 11.98 & 73 & 55.0 & 12.97 \\ 11: 27: 14: 32 & 21 & 24.9 & 70.0 & 12.69 & 85 & 54.9 & 12.97 \\ 11: 27: 15: 31 & 21 & 24.9 & 70.0 & 13.38 & 92 & 54.9 & 12.59 \\ 11: 27: 16: 30 & 21 & 24.9 & 70.0 & 14.03 & 103 & 54.9 & 12.59 \\ 11: 27: 17: 29 & 21 & 24.9 & 70.0 & 14.67 & 127 & 54.8 & 13.08 \\ 11: 27: 18: 28 & 21 & 24.7 & 70.0 & 15.29 & 148 & 54.8 & 13.08 \\ 11: 27: 19: 26 & 21 & 24.7 & 70.0 & 15.84 & 163 & 54.7 & 13.44 \\ 11: 27: 20: 25 & 21 & 25.1 & 70.0 & 16.41 & 175 & 54.5 & 13.44 \\ 11: 27: 21: 24 & 21 & 24.9 & 70.0 & 17.01 & 181 & 54.5 & 12.52 \\ 11: 27: 22: 23 & 21 & 24.9 & 70.0 & 17.53 & 197 & 54.4 & 12.52 \\ 11: 27: 23: 22 & 21 & 24.9 & 70.0 & 17.98 & 225 & 54.4 & 13.35 \\ 11: 27: 24: 21 & 21 & 25.1 & 70.0 & 18.47 & 267 & 54.4 & 13.35 \\ 11: 27: 25: 20 & 21 & 25.1 & 70.0 & 18.94 & 240 & 54.3 & 13.00 \\ 11: 27: 26: 18 & 21 & 25.3 & 70.0 & 19.19 & 437 & 54.3 & 13.00\end{array}$


N10772.TXT

HNF-3557

Rev. 0

100IVD/8_IN_UREA_SN_N10772_750LB_55RPM_30SCFM_TO_COMPARE_NOFLOW_INSERT

TIME PURG_TC2 PurgePSI PurgeCFM DEPTH(IN) DwnFrc(lb) RPM Inches/Min

$\begin{array}{lccccccc}17: 06: 32: 29 & 21 & -0.6 & 2.1 & -0.00 & 1 & 55.5 & -0.32 \\ \text { 17:06:33:22 } & 21 & 9.7 & 54.0 & 0.57 & 1 & 55.5 & -0.32 \\ \text { 17:06:34:21 } & 21 & 24.5 & 54.1 & 1.35 & 1 & 55.3 & 5.52 \\ 17: 06: 35: 20 & 21 & 24.7 & 54.1 & 2.14 & 4 & 55.3 & 5.52 \\ 17: 06: 36: 19 & 21 & 24.7 & 54.1 & 2.93 & 7 & 54.6 & 11.95 \\ 17: 06: 37: 18 & 21 & 24.9 & 54.1 & 3.70 & 9 & 54.5 & 11.95 \\ 17: 06: 38: 17 & 21 & 25.1 & 54.1 & 4.47 & 14 & 54.5 & 12.50 \\ 17: 06: 39: 16 & 21 & 25.1 & 54.1 & 5.23 & 15 & 54.5 & 12.50 \\ 17: 06: 40: 14 & 21 & 24.9 & 54.1 & 6.00 & 18 & 54.4 & 12.37 \\ 17: 06: 41: 13 & 21 & 25.1 & 54.1 & 6.77 & 23 & 54.4 & 12.85 \\ 17: 06: 42: 12 & 21 & 25.1 & 54.1 & 7.51 & 27 & 54.4 & 12.85 \\ 17: 06: 43: 11 & 21 & 25.1 & 54.1 & 8.29 & 31 & 54.5 & 11.78 \\ 17: 06: 44: 10 & 21 & 25.3 & 54.1 & 9.03 & 33 & 54.5 & 11.78 \\ 17: 06: 45: 09 & 21 & 25.3 & 54.1 & 9.79 & 35 & 54.5 & 8.71 \\ 17: 06: 46: 08 & 21 & 25.3 & 54.1 & 10.52 & 39 & 54.4 & 8.71 \\ 17: 06: 47: 06 & 21 & 25.3 & 54.1 & 11.23 & 43 & 54.4 & 7.92 \\ 17: 06: 48: 05 & 21 & 25.3 & 54.1 & 12.01 & 47 & 54.4 & 7.92 \\ 17: 06: 49: 04 & 21 & 25.3 & 54.1 & 12.72 & 49 & 54.3 & 12.12 \\ 17: 06: 50: 03 & 21 & 25.5 & 54.1 & 13.46 & 52 & 54.3 & 12.12 \\ 17: 06: 51: 02 & 21 & 25.5 & 54.1 & 14.16 & 52 & 54.3 & 11.89 \\ 17: 06: 52: 01 & 21 & 25.5 & 54.1 & 14.87 & 56 & 54.3 & 11.89 \\ 17: 06: 53: 00 & 21 & 25.7 & 54.1 & 15.59 & 59 & 54.3 & 12.08 \\ 17: 06: 53: 99 & 21 & 25.7 & 54.1 & 16.28 & 64 & 54.3 & 12.08 \\ 17: 06: 54: 97 & 21 & 25.5 & 54.1 & 16.97 & 67 & 54.3 & 12.63 \\ 17: 06: 55: 96 & 21 & 25.5 & 54.1 & 17.67 & 69 & 54.3 & 12.63 \\ 17: 06: 56: 95 & 21 & 25.7 & 54.1 & 18.36 & 71 & 54.2 & 15.02 \\ 17: 06: 57: 94 & 21 & 25.7 & 54.1 & 19.01 & 74 & 54.2 & 15.02\end{array}$


010772.TXT

HNF-3557

Rev. 0

100IVD/8_IN_DRY_UREA_SN_O10772_750LB_55RPM_30SCFM_TO_COMPARE_NOFLOW_INSERT

TIME PURG_TC2 PurgePSI PurgeCFM DEPTH(IN) DwnFrc(b) RPM Inches/Min

$\begin{array}{llllllll}17: 14: 10: 48 & 21 & -0.6 & 2.2 & 0.10 & -0 & 55.5 & -0.80 \\ 17: 14: 11: 47 & 21 & 20.8 & 2.2 & 0.79 & 1 & 55.5 & -0.80 \\ 17: 14: 12: 46 & 21 & 25.1 & 2.2 & 1.58 & 1 & 55.5 & 10.53 \\ 17: 14: 13: 44 & 21 & 25.1 & 2.2 & 2.38 & 3 & 54.8 & 10.53 \\ 17: 14: 14: 43 & 21 & 25.1 & 2.2 & 3.16 & 5 & 54.5 & 12.04 \\ 17: 14: 15: 42 & 21 & 25.1 & 2.2 & 3.95 & 6 & 54.5 & 12.04 \\ 17: 14: 16: 41 & 21 & 25.1 & 2.2 & 4.73 & 8 & 54.4 & 12.89 \\ 17: 14: 17: 40 & 21 & 25.3 & 2.2 & 5.51 & 10 & 54.4 & 12.89 \\ 17: 14: 18: 39 & 21 & 25.3 & 2.2 & 6.28 & 11 & 54.4 & 12.69 \\ 17: 14: 19: 38 & 21 & 25.3 & 2.2 & 7.06 & 11 & 54.4 & 12.69 \\ 17: 14: 20: 36 & 21 & 25.3 & 2.2 & 7.81 & 14 & 54.4 & 12.40 \\ 17: 14: 21: 35 & 21 & 25.3 & 2.2 & 8.59 & 16 & 54.4 & 12.40 \\ 17: 14: 22: 34 & 21 & 25.3 & 2.2 & 9.38 & 18 & 54.5 & 11.06 \\ 17: 14: 23: 33 & 21 & 25.5 & 2.2 & 10.12 & 21 & 54.5 & 11.06 \\ 17: 14: 24: 32 & 21 & 25.5 & 2.2 & 10.89 & 23 & 54.5 & 7.96 \\ 17: 14: 25: 31 & 21 & 25.5 & 2.2 & 11.67 & 26 & 54.5 & 7.96 \\ 17: 14: 26: 30 & 21 & 25.7 & 2.2 & 12.40 & 29 & 54.6 & 11.28 \\ 17: 14: 27: 29 & 21 & 25.5 & 2.2 & 13.17 & 30 & 54.6 & 11.28 \\ 17: 14: 28: 27 & 21 & 25.7 & 2.2 & 13.97 & 34 & 54.6 & 13.60 \\ 17: 14: 29: 26 & 21 & 25.7 & 2.3 & 14.67 & 41 & 54.5 & 13.60 \\ 17: 14: 30: 25 & 21 & 25.7 & 2.3 & 15.43 & 48 & 54.5 & 14.04 \\ 17: 14: 31: 24 & 21 & 25.7 & 2.3 & 16.15 & 56 & 54.4 & 14.04 \\ 17: 14: 32: 23 & 21 & 25.7 & 2.3 & 16.83 & 60 & 54.4 & 13.58 \\ 17: 14: 33: 22 & 21 & 25.7 & 2.3 & 17.57 & 70 & 54.4 & 13.58 \\ 17: 14: 34: 21 & 21 & 25.7 & 2.3 & 18.22 & 81 & 54.4 & 13.39 \\ 17: 14: 35: 19 & 21 & 25.9 & 2.3 & 18.87 & 95 & 54.4 & 13.39\end{array}$


P10772.TXT

100IVD/8_IN_DRY_UREA_SN_P10772_750LB_55RPM_30SCFM_TO_COMPARE_NOFLOW_INSERT

TIME PURG_TC2 PurgePSI PurgeCFM DEPTH(IN) DwnFrc(Ib) RPM Inches/Min

$\begin{array}{lccccccc}17: 19: 54: 20 & 21 & -0.6 & 2.3 & 0.15 & -1 & 55.6 & -1.15 \\ 17: 19: 55: 19 & 21 & 9.5 & 2.3 & 0.57 & -0 & 55.6 & -1.15 \\ 17: 19: 56: 18 & 21 & 24.5 & 2.3 & 1.36 & 1 & 55.6 & 8.36 \\ 17: 19: 57: 17 & 21 & 25.5 & 2.3 & 2.17 & 1 & 55.2 & 8.36 \\ 17: 19: 58: 16 & 21 & 25.5 & 2.3 & 2.94 & 3 & 54.7 & 12.76 \\ 17: 19: 59: 15 & 21 & 25.7 & 2.3 & 3.73 & 5 & 54.7 & 12.76 \\ 17: 20: 00: 13 & 21 & 25.7 & 2.3 & 4.53 & 6 & 54.6 & 13.08 \\ 17: 20: 01: 12 & 21 & 25.9 & 2.3 & 5.33 & 6 & 54.6 & 13.08 \\ 17: 20: 02: 11 & 21 & 25.9 & 2.3 & 6.11 & 9 & 54.6 & 12.46 \\ 17: 20: 03: 10 & 21 & 26.1 & 2.3 & 6.87 & 11 & 54.6 & 12.46 \\ 17: 20: 04: 09 & 21 & 25.9 & 2.3 & 7.66 & 13 & 54.6 & 13.49 \\ 17: 20: 05: 08 & 21 & 26.1 & 2.3 & 8.43 & 14 & 54.6 & 13.49 \\ 17: 20: 06: 07 & 22 & 26.1 & 2.3 & 9.23 & 17 & 54.6 & 11.12 \\ 17: 20: 07: 05 & 21 & 26.1 & 2.3 & 9.98 & 18 & 54.6 & 11.12 \\ 17: 20: 08: 04 & 21 & 26.3 & 2.3 & 10.76 & 19 & 54.6 & 9.12 \\ 17: 20: 09: 03 & 21 & 26.3 & 2.3 & 11.52 & 22 & 54.6 & 9.12 \\ 17: 20: 10: 02 & 21 & 26.1 & 2.3 & 12.28 & 25 & 54.6 & 10.67 \\ 17: 20: 11: 01 & 21 & 26.3 & 2.3 & 13.03 & 29 & 54.6 & 10.67 \\ 17: 20: 12: 00 & 21 & 26.1 & 2.3 & 13.79 & 37 & 54.5 & 13.99 \\ 17: 20: 12: 99 & 21 & 26.3 & 2.3 & 14.55 & 40 & 54.7 & 13.99 \\ 17: 20: 13: 98 & 21 & 26.3 & 2.3 & 15.27 & 43 & 54.7 & 13.62 \\ 17: 20: 14: 96 & 21 & 26.5 & 2.3 & 16.01 & 52 & 54.6 & 13.62 \\ 17: 20: 15: 95 & 21 & 26.3 & 2.3 & 16.70 & 63 & 54.6 & 12.89 \\ 17: 20: 16: 94 & 21 & 26.3 & 2.3 & 17.43 & 72 & 54.6 & 12.89 \\ 17: 20: 17: 93 & 21 & 26.5 & 2.3 & 18.10 & 81 & 54.6 & 13.70 \\ 17: 20: 18: 92 & 21 & 26.6 & 2.3 & 18.70 & 96 & 54.4 & 13.70 \\ 17: 20: 19: 91 & 21 & 26.6 & 2.3 & 19.32 & 104 & 54.4 & 15.73 \\ 17: 20: 20: 90 & 21 & 22.8 & 2.3 & 19.32 & 49 & 54.4 & 15.73\end{array}$


100IVD/9_IN_DRY_UREA_SN_K_NFI_750LB_55RPM_30SCFM_TO_COMPARE_STANDARD_INSERT TIME PURG_TC2 PurgePSI PurgeCFM DEPTH(IN) DwnFrc(lb) RPM inches/Min

$\begin{array}{lccccccc}18: 31: 35: 80 & 22 & -0.6 & -5.6 & 0.01 & 3 & 47.6 & -1.15 \\ 18: 31: 36: 79 & 21 & 4.4 & 60.0 & 0.65 & 3 & 47.6 & -1.15 \\ 18: 31: 37: 78 & 22 & 22.8 & 57.3 & 1.49 & 4 & 49.5 & 8.34 \\ 18: 31: 38: 77 & 22 & 22.9 & 57.0 & 2.27 & 5 & 50.6 & 8.34 \\ 18: 31: 39: 76 & 22 & 22.9 & 56.9 & 3.07 & 6 & 50.6 & 12.24 \\ 18: 31: 40: 75 & 22 & 22.9 & 56.7 & 3.83 & 8 & 51.9 & 12.24 \\ 18: 31: 41: 79 & 22 & 22.9 & 56.7 & 4.67 & 9 & 52.9 & 12.82 \\ 18: 31: 42: 78 & 22 & 22.9 & 56.7 & 5.52 & 11 & 52.9 & 12.82 \\ 18: 31: 43: 77 & 22 & 23.1 & 56.7 & 6.29 & 13 & 53.7 & 12.25 \\ 18: 31: 44: 81 & 22 & 23.1 & 56.5 & 7.11 & 15 & 54.1 & 12.25 \\ 18: 31: 45: 80 & 22 & 23.1 & 56.5 & 7.95 & 17 & 54.1 & 13.43 \\ 18: 31: 46: 79 & 22 & 23.1 & 56.4 & 8.72 & 21 & 54.4 & 13.43 \\ 18: 31: 47: 83 & 22 & 23.3 & 56.4 & 9.53 & 28 & 54.6 & 10.84 \\ 18: 31: 48: 82 & 22 & 23.5 & 56.4 & 10.32 & 24 & 54.6 & 8.74 \\ 18: 31: 49: 81 & 22 & 23.5 & 56.3 & 11.04 & 29 & 54.7 & 8.74 \\ 18: 31: 50: 80 & 22 & 23.5 & 56.3 & 11.84 & 34 & 54.7 & 11.17 \\ 18: 31: 51: 79 & 22 & 23.9 & 56.3 & 12.58 & 42 & 54.7 & 11.17 \\ 18: 31: 52: 83 & 22 & 24.1 & 56.2 & 13.38 & 47 & 54.8 & 13.13 \\ 18: 31: 53: 82 & 22 & 24.5 & 56.1 & 14.12 & 58 & 54.8 & 13.13 \\ 18: 31: 54: 81 & 22 & 24.9 & 55.9 & 14.82 & 62 & 54.8 & 12.79 \\ 18: 31: 55: 85 & 22 & 25.1 & 55.8 & 15.60 & 72 & 54.7 & 12.79 \\ 18: 31: 56: 84 & 22 & 25.7 & 55.0 & 16.32 & 91 & 54.6 & 13.25 \\ 18: 31: 57: 83 & 22 & 26.5 & 54.4 & 16.95 & 98 & 54.6 & 13.25 \\ 18: 31: 58: 82 & 21 & 26.8 & 54.2 & 17.64 & 115 & 54.6 & 14.10 \\ 18: 31: 59: 80 & 22 & 27.8 & 53.7 & 18.23 & 134 & 54.6 & 14.10 \\ 18: 32: 00: 85 & 22 & 28.8 & 53.3 & 18.81 & 141 & 54.6 & 13.97 \\ 18: 32: 01: 84 & 22 & 29.0 & 53.0 & 19.39 & 144 & 54.6 & 13.97\end{array}$


100IVD/9_IN_DRY_UREA_SN_L_NFI_750LB_55RPM_30SCFM_TO_COMPARE_STANDARD_INSERT

TIME PURG_TC2 PurgePSI PurgeCFM DEPTH(IN) DwnFrc(lb) RPM Inches/Min

$\begin{array}{lccccccc}19: 04: 15: 43 & 21 & -0.6 & -5.1 & -0.02 & 6 & 55.1 & -1.67 \\ 19: 04: 16: 42 & 22 & 5.2 & 57.7 & 0.39 & 5 & 55.3 & -1.44 \\ 19: 04: 17: 41 & 22 & 21.4 & 56.3 & 1.14 & 7 & 55.3 & -1.44 \\ 19: 04: 18: 45 & 22 & 21.6 & 55.9 & 1.97 & 8 & 55.0 & 10.24 \\ 19: 04: 19: 44 & 22 & 21.6 & 55.8 & 2.79 & 9 & 54.4 & 12.41 \\ 19: 04: 20: 43 & 22 & 21.6 & 55.8 & 3.54 & 12 & 54.4 & 12.41 \\ 19: 04: 21: 42 & 22 & 21.8 & 55.8 & 4.34 & 14 & 54.4 & 12.19 \\ 19: 04: 22: 41 & 22 & 21.8 & 55.7 & 5.11 & 16 & 54.4 & 12.19 \\ 19: 04: 23: 45 & 22 & 21.8 & 55.7 & 5.95 & 18 & 54.4 & 12.16 \\ 19: 04: 24: 44 & 22 & 22.0 & 55.7 & 6.75 & 21 & 54.4 & 12.16 \\ 19: 04: 25: 43 & 22 & 22.0 & 55.6 & 7.49 & 22 & 54.5 & 12.58 \\ 19: 04: 26: 47 & 22 & 22.0 & 55.6 & 8.30 & 24 & 54.5 & 12.58 \\ 19: 04: 27: 46 & 22 & 22.2 & 55.5 & 9.09 & 29 & 54.5 & 10.39 \\ 19: 04: 28: 45 & 22 & 22.2 & 55.5 & 9.85 & 29 & 54.5 & 10.39 \\ 19: 04: 29: 44 & 22 & 22.2 & 55.5 & 10.60 & 34 & 54.5 & 8.31 \\ 19: 04: 30: 43 & 22 & 22.4 & 55.4 & 11.33 & 41 & 54.5 & 8.31 \\ 19: 04: 31: 47 & 22 & 22.6 & 54.9 & 12.10 & 49 & 54.6 & 11.83 \\ 19: 04: 32: 46 & 22 & 23.1 & 54.3 & 12.83 & 51 & 54.6 & 11.83 \\ 19: 04: 33: 45 & 22 & 23.1 & 54.2 & 13.56 & 54 & 54.7 & 12.91 \\ 19: 04: 34: 44 & 22 & 23.5 & 54.1 & 14.29 & 57 & 54.5 & 12.91 \\ 19: 04: 35: 43 & 22 & 23.7 & 54.0 & 15.02 & 66 & 54.5 & 13.32 \\ 19: 04: 36: 47 & 22 & 24.3 & 53.7 & 15.73 & 75 & 54.4 & 13.32 \\ 19: 04: 37: 46 & 22 & 24.5 & 53.3 & 16.40 & 84 & 54.4 & 12.30 \\ 19: 04: 38: 45 & 22 & 23.1 & 53.2 & 17.04 & 118 & 54.4 & 12.30 \\ 19: 04: 39: 49 & 22 & 23.3 & 53.2 & 17.65 & 147 & 54.3 & 13.82 \\ 19: 04: 40: 48 & 22 & 23.5 & 53.2 & 18.22 & 169 & 54.2 & 13.82 \\ 19: 04: 41: 47 & 22 & 23.7 & 53.2 & 18.74 & 182 & 54.2 & 14.27 \\ 19: 04: 42: 46 & 22 & 23.7 & 53.2 & 19.26 & 192 & 54.1 & 14.27\end{array}$


TIME PURG_TC2 PurgePSI PurgeCFM DEPTH(IN) DwnFrc(ib) RPM Inches/Min

$\begin{array}{lllllllc}19: 12: 31: 96 & 22 & -0.6 & -5.7 & -0.00 & 4 & 55.5 & -0.54 \\ 19: 12: 32: 95 & 22 & 19.6 & 56.7 & 0.42 & 4 & 55.6 & 2.16 \\ 19: 12: 33: 94 & 22 & 21.4 & 55.9 & 1.23 & 6 & 55.6 & 2.16 \\ 19: 12: 34: 93 & 22 & 21.6 & 55.8 & 2.04 & 9 & 55.1 & 12.22 \\ 19: 12: 35: 91 & 22 & 21.6 & 55.7 & 2.85 & 8 & 54.6 & 12.22 \\ 19: 12: 36: 90 & 22 & 21.6 & 55.7 & 3.64 & 10 & 54.6 & 12.22 \\ 19: 12: 37: 89 & 22 & 21.8 & 55.6 & 4.42 & 12 & 54.4 & 12.22 \\ 19: 12: 38: 88 & 22 & 21.8 & 55.6 & 5.22 & 14 & 54.4 & 11.95 \\ 19: 12: 39: 87 & 22 & 21.8 & 55.5 & 6.01 & 14 & 54.4 & 11.95 \\ 19: 12: 40: 86 & 22 & 21.8 & 55.5 & 6.79 & 16 & 54.4 & 12.88 \\ 19: 12: 41: 85 & 22 & 22.0 & 55.5 & 7.59 & 17 & 54.4 & 12.88 \\ 19: 12: 42: 84 & 22 & 22.0 & 55.5 & 8.37 & 19 & 54.4 & 12.32 \\ 19: 12: 43: 82 & 22 & 22.2 & 55.5 & 9.16 & 22 & 54.3 & 12.32 \\ 19: 12: 44: 81 & 22 & 22.4 & 55.4 & 9.93 & 26 & 54.3 & 8.66 \\ 19: 12: 45: 80 & 22 & 22.2 & 55.4 & 10.69 & 28 & 54.3 & 8.66 \\ 19: 12: 46: 79 & 22 & 22.6 & 55.4 & 11.46 & 32 & 54.3 & 8.38 \\ 19: 12: 47: 78 & 22 & 22.8 & 54.9 & 12.21 & 39 & 54.4 & 8.38 \\ 19: 12: 48: 77 & 22 & 23.1 & 54.5 & 12.98 & 47 & 54.4 & 13.84 \\ 19: 12: 49: 76 & 22 & 23.1 & 54.4 & 13.71 & 58 & 54.4 & 13.84 \\ 19: 12: 50: 74 & 22 & 23.9 & 54.1 & 14.44 & 67 & 54.4 & 12.92 \\ 19: 12: 51: 73 & 22 & 24.3 & 54.0 & 15.14 & 69 & 54.4 & 12.92 \\ 19: 12: 52: 72 & 22 & 24.7 & 53.7 & 15.84 & 82 & 54.4 & 12.72 \\ 19: 12: 53: 71 & 22 & 25.1 & 53.4 & 16.48 & 93 & 54.4 & 12.72 \\ 19: 12: 54: 70 & 22 & 26.3 & 52.9 & 17.17 & 113 & 54.4 & 12.71 \\ 19: 12: 55: 69 & 22 & 27.0 & 52.4 & 17.81 & 132 & 54.6 & 12.71 \\ 19: 12: 56: 68 & 22 & 27.2 & 52.1 & 18.37 & 152 & 54.6 & 12.93 \\ 19: 12: 57: 67 & 22 & 28.0 & 51.8 & 18.92 & 163 & 54.6 & 12.93\end{array}$


100IVD/9_IN_DRY_KMAG_SN_N_NFI_750LB_55RPM_30SCFM_TO_COMPARE_STANDARD_INSERT

TIME PURG_TC2 PurgePSI PurgeCFM DEPTH(IN) DwnFrc(Ib) RPM Inches/Min

$\begin{array}{lllllllc}19: 22: 36: 47 & 22 & -0.8 & -5.4 & 0.01 & 109 & 55.8 & -0.51 \\ 19: 22: 37: 40 & 22 & 15.7 & 56.6 & 0.77 & 116 & 55.6 & -0.51 \\ 19: 22: 38: 39 & 22 & 21.8 & 56.2 & 1.56 & 118 & 55.6 & 6.96 \\ 19: 22: 39: 38 & 22 & 22.0 & 56.2 & 2.35 & 126 & 55.0 & 6.96 \\ 19: 22: 40: 37 & 22 & 22.2 & 56.2 & 3.13 & 135 & 54.7 & 12.61 \\ 19: 22: 41: 36 & 22 & 22.2 & 55.8 & 3.90 & 136 & 54.7 & 12.61 \\ 19: 22: 42: 35 & 22 & 22.4 & 55.6 & 4.66 & 149 & 54.8 & 12.36 \\ 19: 22: 43: 34 & 22 & 22.6 & 55.6 & 5.43 & 162 & 54.7 & 12.36 \\ 19: 22: 44: 32 & 22 & 22.8 & 55.4 & 6.15 & 174 & 54.7 & 11.99 \\ 19: 22: 45: 31 & 22 & 22.9 & 54.7 & 6.87 & 187 & 54.5 & 12.28 \\ 19: 22: 46: 30 & 22 & 23.1 & 54.5 & 7.57 & 202 & 54.5 & 12.28 \\ 19: 22: 47: 29 & 22 & 23.5 & 54.3 & 8.27 & 210 & 54.5 & 10.62 \\ 19: 22: 48: 28 & 22 & 23.5 & 54.1 & 8.95 & 220 & 54.4 & 10.62 \\ 19: 22: 49: 27 & 22 & 23.9 & 54.0 & 9.61 & 234 & 54.4 & 7.86 \\ 19: 22: 50: 26 & 22 & 24.3 & 53.9 & 10.26 & 248 & 54.4 & 7.86 \\ 19: 22: 51: 24 & 22 & 25.3 & 53.4 & 10.86 & 267 & 54.4 & 6.20 \\ 19: 22: 52: 29 & 22 & 25.1 & 53.2 & 11.46 & 286 & 54.5 & 6.20 \\ 19: 22: 53: 28 & 22 & 26.5 & 52.8 & 12.03 & 297 & 54.5 & 10.51 \\ 19: 22: 54: 27 & 22 & 27.2 & 52.2 & 12.53 & 310 & 54.5 & 10.51 \\ 19: 22: 55: 25 & 22 & 27.6 & 52.0 & 13.10 & 319 & 54.5 & 10.75 \\ 19: 22: 56: 24 & 22 & 27.4 & 51.8 & 13.60 & 341 & 54.5 & 10.75 \\ 19: 22: 57: 29 & 22 & 27.8 & 51.8 & 14.11 & 354 & 54.5 & 11.07 \\ 19: 22: 58: 28 & 22 & 28.2 & 51.3 & 14.61 & 367 & 54.4 & 11.07 \\ 19: 22: 59: 26 & 22 & 28.4 & 51.2 & 15.03 & 377 & 54.4 & 10.14 \\ 19: 23: 00: 25 & 22 & 28.4 & 51.2 & 15.44 & 402 & 54.3 & 10.14 \\ 19: 23: 01: 24 & 22 & 28.8 & 51.1 & 15.79 & 416 & 54.3 & 8.78 \\ 19: 23: 02: 28 & 22 & 29.6 & 50.7 & 16.16 & 422 & 54.3 & 8.78 \\ 19: 23: 03: 27 & 22 & 30.7 & 50.4 & 16.52 & 438 & 54.2 & 10.33 \\ 19: 23: 04: 26 & 22 & 31.1 & 50.1 & 16.84 & 471 & 54.3 & 10.33 \\ 19: 23: 05: 25 & 22 & 31.9 & 49.8 & 17.17 & 473 & 54.3 & 8.21 \\ 19: 23: 06: 24 & 22 & 32.9 & 49.7 & 17.49 & 476 & 54.4 & 8.21 \\ 19: 23: 07: 28 & 22 & 33.5 & 49.2 & 17.78 & 469 & 54.4 & 8.18 \\ 19: 23: 08: 27 & 22 & 34.2 & 48.8 & 18.09 & 467 & 54.4 & 8.18 \\ 19: 23: 09: 26 & 22 & 34.4 & 48.6 & 18.38 & 440 & 54.3 & 8.70 \\ 19: 23: 10: 30 & 22 & 33.7 & 48.4 & 18.70 & 427 & 54.3 & 9.52 \\ 19: 23: 11: 29 & 22 & 32.9 & 48.4 & 19.04 & 408 & 54.3 & 9.52\end{array}$


100IVD/9_IN_DRY_KMAG_SN_O_NFI_750LB_55RPM_3OSCFM_TO_COMPARE_STANDARD_INSERT

TIME PURG_TC2 PurgePSI PurgeCFM DEPTH(IN) DwnFrc(lb) RPM Inches/Min

$\begin{array}{llllllll}19: 53: 17: 63 & 22 & -0.6 & -5.5 & 0.01 & -1 & 55.5 & -1.10 \\ 19: 53: 18: 56 & 22 & 20.8 & 56.2 & 0.71 & 2 & 55.6 & -1.10 \\ 19: 53: 19: 55 & 22 & 22.0 & 55.9 & 1.47 & 4 & 55.6 & 10.02 \\ 19: 53: 20: 54 & 22 & 22.0 & 55.8 & 2.30 & 8 & 54.9 & 10.02 \\ 19: 53: 21: 53 & 22 & 22.2 & 55.8 & 3.09 & 14 & 54.5 & 12.71 \\ 19: 53: 22: 57 & 22 & 22.4 & 55.6 & 3.88 & 20 & 54.5 & 12.71 \\ 19: 53: 23: 56 & 22 & 22.4 & 55.5 & 4.70 & 32 & 54.5 & 12.80 \\ 19: 53: 24: 55 & 22 & 22.4 & 55.5 & 5.48 & 44 & 54.5 & 12.80 \\ 19: 53: 25: 59 & 22 & 22.8 & 55.4 & 6.22 & 57 & 54.5 & 12.23 \\ 19: 53: 26: 58 & 22 & 22.8 & 55.1 & 6.98 & 66 & 54.5 & 12.23 \\ 19: 53: 27: 57 & 22 & 23.1 & 54.7 & 7.66 & 84 & 54.6 & 11.92 \\ 19: 53: 28: 56 & 22 & 23.5 & 54.2 & 8.40 & 88 & 54.6 & 11.92 \\ 19: 53: 29: 55 & 21 & 24.3 & 53.7 & 9.09 & 103 & 54.6 & 10.18 \\ 19: 53: 30: 53 & 22 & 24.7 & 53.4 & 9.74 & 111 & 54.6 & 10.18 \\ 19: 53: 31: 52 & 22 & 25.1 & 53.2 & 10.40 & 130 & 54.6 & 7.16 \\ 19: 53: 32: 51 & 22 & 25.1 & 53.0 & 11.01 & 138 & 54.5 & 7.16 \\ 19: 53: 33: 50 & 22 & 25.1 & 53.0 & 11.66 & 152 & 54.5 & 7.05 \\ 19: 53: 34: 49 & 22 & 25.7 & 52.8 & 12.20 & 171 & 54.5 & 7.05 \\ 19: 53: 35: 48 & 22 & 26.8 & 52.0 & 12.81 & 182 & 54.5 & 12.09 \\ 19: 53: 36: 47 & 22 & 27.4 & 51.6 & 13.36 & 201 & 54.4 & 12.09 \\ 19: 53: 37: 46 & 22 & 27.8 & 51.4 & 13.87 & 216 & 54.4 & 12.34 \\ 19: 53: 38: 44 & 22 & 28.0 & 51.2 & 14.36 & 220 & 54.3 & 12.34 \\ 19: 53: 39: 49 & 22 & 28.6 & 50.9 & 14.90 & 246 & 54.3 & 10.71 \\ 19: 53: 40: 48 & 22 & 30.2 & 49.8 & 15.41 & 279 & 54.3 & 10.42 \\ 19: 53: 41: 47 & 22 & 31.3 & 49.5 & 15.86 & 294 & 54.2 & 10.42 \\ 19: 53: 42: 51 & 22 & 32.1 & 49.2 & 16.28 & 300 & 54.2 & 11.39 \\ 19: 53: 43: 50 & 22 & 33.1 & 48.6 & 16.72 & 309 & 54.2 & 11.39 \\ 19: 53: 44: 49 & 22 & 33.9 & 48.2 & 17.13 & 323 & 54.2 & 11.47 \\ 19: 53: 45: 47 & 22 & 34.2 & 47.5 & 17.54 & 328 & 54.1 & 11.47 \\ 19: 53: 46: 46 & 22 & 33.7 & 47.4 & 17.97 & 331 & 54.1 & 11.53 \\ 19: 53: 47: 51 & 22 & 32.7 & 47.4 & 18.38 & 329 & 54.0 & 11.53 \\ 19: 53: 48: 50 & 22 & 32.3 & 47.4 & 18.79 & 315 & 54.1 & 9.44 \\ 19: 53: 49: 48 & 22 & 29.8 & 47.6 & 19.21 & 294 & 54.1 & 9.44\end{array}$


P_NFI.TXT

HNF-3557

Rev. 0

100IVD/9_IN_DRY_KMAG_SN_P_NFI_750LB_55RPM_30SCFM_TO_COMPARE_STANDARD_INSERT

TIME PURG_TC2 PurgePSI PurgeCFM DEPTH(IN) DwnFrc(lb) RPM Inches/Min

$\begin{array}{lccccccc}20: 01: 45: 58 & 22 & -0.6 & -5.7 & -0.00 & -1 & 55.8 & -1.08 \\ 20: 01: 46: 51 & 22 & 9.5 & 56.1 & 0.62 & -1 & 55.8 & 1.65 \\ 20: 01: 47: 56 & 22 & 21.4 & 55.7 & 1.42 & 2 & 55.7 & 1.65 \\ 20: 01: 48: 54 & 22 & 21.6 & 55.6 & 2.27 & 9 & 54.9 & 12.38 \\ 20: 01: 49: 53 & 22 & 21.8 & 55.4 & 3.03 & 15 & 54.9 & 12.38 \\ 20: 01: 50: 52 & 22 & 21.8 & 55.2 & 3.86 & 24 & 54.6 & 12.95 \\ 20: 01: 51: 51 & 22 & 22.0 & 54.8 & 4.61 & 34 & 54.6 & 12.95 \\ 20: 01: 52: 55 & 22 & 22.0 & 54.7 & 5.43 & 45 & 54.6 & 12.32 \\ 20: 01: 53: 54 & 22 & 22.4 & 54.5 & 6.20 & 56 & 54.7 & 12.32 \\ 20: 01: 54: 53 & 22 & 22.6 & 54.2 & 6.92 & 67 & 54.7 & 12.53 \\ 20: 01: 55: 57 & 22 & 22.6 & 54.1 & 7.66 & 81 & 54.7 & 12.53 \\ 20: 01: 56: 56 & 22 & 22.6 & 54.0 & 8.40 & 94 & 54.7 & 11.07 \\ 20: 01: 57: 55 & 22 & 23.1 & 53.9 & 9.07 & 110 & 54.6 & 11.07 \\ 20: 01: 58: 54 & 22 & 23.9 & 53.5 & 9.76 & 119 & 54.6 & 8.65 \\ 20: 01: 59: 53 & 22 & 23.9 & 53.3 & 10.39 & 131 & 54.6 & 8.65 \\ 20: 02: 00: 57 & 22 & 24.3 & 53.0 & 11.01 & 146 & 54.6 & 6.33 \\ 20: 02: 01: 56 & 22 & 24.9 & 52.9 & 11.65 & 164 & 54.6 & 9.23 \\ 20: 02: 02: 55 & 22 & 25.3 & 52.7 & 12.20 & 181 & 54.6 & 9.23 \\ 20: 02: 03: 59 & 22 & 25.7 & 52.3 & 12.76 & 198 & 54.6 & 12.26 \\ 20: 02: 04: 58 & 22 & 25.7 & 52.2 & 13.36 & 219 & 54.6 & 12.26 \\ 20: 02: 05: 57 & 22 & 26.1 & 52.1 & 13.85 & 239 & 54.5 & 12.09 \\ 20: 02: 06: 56 & 22 & 26.3 & 52.0 & 14.34 & 246 & 54.4 & 12.09 \\ 20: 02: 07: 55 & 22 & 26.6 & 51.6 & 14.82 & 254 & 54.4 & 10.12 \\ 20: 02: 08: 59 & 22 & 27.2 & 51.2 & 15.24 & 268 & 54.5 & 10.12 \\ 20: 02: 09: 58 & 22 & 28.2 & 50.7 & 15.70 & 274 & 54.3 & 10.13 \\ 20: 02: 10: 57 & 22 & 29.4 & 49.9 & 16.11 & 276 & 54.3 & 10.13 \\ 20: 02: 11: 56 & 22 & 31.1 & 49.2 & 16.48 & 323 & 54.3 & 10.29 \\ 20: 02: 12: 55 & 22 & 32.1 & 48.4 & 16.85 & 346 & 54.3 & 10.29 \\ 20: 02: 13: 54 & 22 & 33.7 & 47.3 & 17.21 & 352 & 54.3 & 10.65 \\ 20: 02: 14: 52 & 22 & 35.2 & 46.4 & 17.58 & 346 & 54.1 & 10.65 \\ 20: 02: 15: 51 & 22 & 35.8 & 46.2 & 17.92 & 344 & 54.0 & 10.45 \\ 20: 02: 16: 50 & 22 & 35.6 & 45.9 & 18.25 & 334 & 54.0 & 10.45 \\ 20: 02: 17: 49 & 22 & 34.2 & 45.9 & 18.65 & 319 & 54.0 & 11.00 \\ 20: 02: 18: 48 & 22 & 32.7 & 45.8 & 19.06 & 300 & 54.0 & 11.00 \\ 20: 02: 19: 47 & 22 & 29.4 & 47.3 & 19.30 & 292 & 54.0 & 11.94 \\ & & & & & & & \end{array}$


Q10772.TXT

HNF-3557

Rev. 0

100IVD/8_IN_DRY_KMAG_SN_Q10772_750LB_55RPM_30SCFM_TO_COMPARE_NO_FLOW_INSERT

TIME PURG_TC2 PurgePSI PurgeCFM DEPTH(IN) DwnFrc(lb) RPM Inches/Min

$\begin{array}{lccccccc}14: 30: 35: 90 & 21 & -0.6 & -5.2 & 0.01 & 4 & 54.2 & -0.44 \\ 14: 30: 36: 89 & 21 & 0.6 & 54.8 & 0.63 & 4 & 54.2 & -0.44 \\ 14: 30: 37: 87 & 21 & 23.7 & 54.9 & 1.41 & 12 & 53.8 & 8.92 \\ 14: 30: 38: 86 & 21 & 24.1 & 54.7 & 2.16 & 17 & 53.8 & 8.92 \\ 14: 30: 39: 85 & 21 & 24.3 & 54.5 & 2.93 & 22 & 53.2 & 13.24 \\ 14: 30: 40: 84 & 21 & 24.3 & 54.4 & 3.71 & 26 & 53.1 & 13.24 \\ 14: 30: 41: 83 & 21 & 24.3 & 54.2 & 4.43 & 37 & 53.1 & 14.13 \\ 14: 30: 42: 82 & 21 & 24.3 & 54.2 & 5.18 & 46 & 53.1 & 14.13 \\ 14: 30: 43: 81 & 21 & 24.3 & 54.2 & 5.92 & 62 & 53.2 & 13.07 \\ 14: 30: 44: 79 & 21 & 24.5 & 54.1 & 6.65 & 70 & 53.2 & 13.07 \\ 14: 30: 45: 78 & 21 & 24.5 & 54.1 & 7.35 & 82 & 53.1 & 13.14 \\ 14: 30: 46: 77 & 21 & 24.7 & 54.1 & 8.07 & 90 & 53.1 & 13.14 \\ 14: 30: 47: 76 & 21 & 24.7 & 54.0 & 8.75 & 108 & 53.1 & 11.63 \\ 14: 30: 48: 75 & 21 & 24.9 & 54.0 & 9.44 & 122 & 53.2 & 11.63 \\ 14: 30: 49: 74 & 21 & 25.1 & 54.0 & 10.11 & 138 & 53.2 & 7.80 \\ 14: 30: 50: 73 & 21 & 25.1 & 54.0 & 10.72 & 153 & 53.2 & 7.80 \\ 14: 30: 51: 72 & 21 & 25.3 & 54.0 & 11.29 & 172 & 53.1 & 6.48 \\ 14: 30: 52: 70 & 21 & 25.3 & 54.0 & 11.88 & 174 & 53.0 & 6.48 \\ 14: 30: 53: 69 & 21 & 25.3 & 54.0 & 12.41 & 195 & 53.0 & 12.28 \\ 14: 30: 54: 68 & 21 & 25.5 & 53.9 & 12.98 & 216 & 52.9 & 12.28 \\ 14: 30: 55: 67 & 21 & 25.9 & 53.8 & 13.50 & 234 & 52.8 & 13.51 \\ 14: 30: 56: 66 & 21 & 26.1 & 53.8 & 14.01 & 254 & 52.8 & 13.51 \\ 14: 30: 57: 65 & 21 & 26.5 & 53.5 & 14.48 & 276 & 52.8 & 13.17 \\ 14: 30: 58: 64 & 21 & 26.8 & 53.2 & 14.93 & 289 & 52.9 & 11.45 \\ 14: 30: 59: 62 & 21 & 27.8 & 53.0 & 15.36 & 300 & 52.9 & 11.45 \\ 14: 31: 00: 61 & 21 & 28.6 & 52.4 & 15.77 & 310 & 53.0 & 9.84 \\ 14: 31: 01: 60 & 21 & 29.8 & 51.4 & 16.16 & 320 & 53.0 & 9.84 \\ 14: 31: 02: 59 & 21 & 31.1 & 50.7 & 16.53 & 349 & 53.0 & 9.67 \\ 14: 31: 03: 58 & 21 & 32.7 & 50.1 & 16.90 & 358 & 52.9 & 9.67 \\ 14: 31: 04: 57 & 21 & 33.3 & 49.6 & 17.25 & 349 & 52.7 & 9.49 \\ 14: 31: 05: 56 & 21 & 35.0 & 48.6 & 17.63 & 341 & 52.7 & 9.49 \\ 14: 31: 06: 55 & 21 & 36.0 & 47.6 & 18.00 & 339 & 52.7 & 10.37 \\ 14: 31: 07: 53 & 21 & 36: 0 & 47.6 & 18.38 & 332 & 52.6 & 10.37 \\ 14: 31: 08: 52 & 21 & 34.6 & 47.5 & 18.74 & 338 & 52.6 & 10.65 \\ 14: 31: 09: 51 & 21 & 33.1 & 47.5 & 19.16 & 317 & 52.6 & 10.65\end{array}$


R10772.TXT

HNF-3557

Rev. 0

100IVD/8_IN_DRY_KMAG_SN_R10772_750LB_55RPM_30SCFM_TO_COMPARE_NO_FLOW_INSERT

TIME PURG_TC2 PurgePSI PurgeCFM DEPTH(IN) DwnFrc(Ib) RPM Inches/Min

$\begin{array}{lccccccc}14: 47: 25: 92 & 21 & -0.6 & -5.6 & 0.01 & 33 & 51.6 & -0.43 \\ 14: 47: 26: 86 & 21 & 8.9 & 55.9 & 0.01 & 36 & 51.6 & -0.43 \\ 14: 47: 27: 84 & 21 & 23.7 & 55.9 & 0.20 & 35 & 52.6 & -0.32 \\ 14: 47: 28: 83 & 21 & 23.7 & 55.9 & 0.99 & 35 & 53.2 & -0.32 \\ 14: 47: 29: 82 & 21 & 23.7 & 55.8 & 1.78 & 42 & 53.2 & 11.62 \\ 14: 47: 30: 81 & 21 & 23.9 & 55.8 & 2.56 & 43 & 52.8 & 11.62 \\ 14: 47: 31: 80 & 21 & 24.3 & 55.8 & 3.35 & 48 & 52.7 & 13.32 \\ 14: 47: 32: 79 & 21 & 24.3 & 55.8 & 4.11 & 49 & 52.7 & 13.32 \\ 14: 47: 33: 78 & 21 & 24.3 & 55.8 & 4.87 & 48 & 52.8 & 13.32 \\ 14: 47: 34: 76 & 21 & 24.5 & 55.8 & 5.66 & 51 & 52.8 & 13.32 \\ 14: 47: 35: 75 & 21 & 24.5 & 55.8 & 6.38 & 53 & 52.8 & 13.87 \\ 14: 47: 36: 74 & 21 & 24.5 & 55.8 & 7.15 & 57 & 52.9 & 13.87 \\ 14: 47: 37: 73 & 21 & 24.9 & 54.8 & 7.88 & 66 & 52.9 & 13.11 \\ 14: 47: 38: 72 & 21 & 24.9 & 54.5 & 8.64 & 69 & 52.9 & 13.11 \\ 14: 47: 39: 71 & 21 & 25.1 & 54.4 & 9.35 & 75 & 52.9 & 11.33 \\ 14: 47: 40: 70 & 21 & 25.3 & 54.2 & 10.12 & 81 & 52.9 & 11.33 \\ 14: 47: 41: 69 & 21 & 25.3 & 54.2 & 10.82 & 87 & 52.9 & 7.98 \\ 14: 47: 42: 67 & 21 & 25.1 & 54.1 & 11.52 & 91 & 52.8 & 7.98 \\ 14: 47: 43: 66 & 21 & 25.1 & 54.1 & 12.18 & 99 & 52.7 & 9.44 \\ 14: 47: 44: 65 & 21 & 25.1 & 54.0 & 12.92 & 105 & 52.7 & 9.44 \\ 14: 47: 45: 64 & 21 & 25.1 & 54.0 & 13.60 & 117 & 52.8 & 14.21 \\ 14: 47: 46: 63 & 21 & 25.1 & 54.0 & 14.26 & 129 & 52.9 & 13.90 \\ 14: 47: 47: 62 & 21 & 25.5 & 54.0 & 14.91 & 139 & 52.9 & 13.90 \\ 14: 47: 48: 61 & 21 & 25.3 & 54.0 & 15.55 & 149 & 52.9 & 12.86 \\ 14: 47: 49: 59 & 21 & 25.5 & 54.0 & 16.16 & 160 & 52.8 & 12.86 \\ 14: 47: 50: 58 & 21 & 25.5 & 54.0 & 16.76 & 166 & 52.8 & 15.09 \\ 14: 47: 51: 57 & 21 & 25.5 & 53.9 & 17.37 & 177 & 52.9 & 15.09 \\ 14: 47: 52: 56 & 21 & 25.5 & 53.9 & 17.93 & 191 & 52.9 & 14.84 \\ 14: 47: 53: 55 & 21 & 25.5 & 53.8 & 18.46 & 191 & 52.9 & 14.84 \\ 14: 47: 54: 54 & 21 & 25.5 & 53.8 & 19.03 & 203 & 53.0 & 13.25 \\ 14: 47: 55: 53 & 21 & 25.5 & 53.8 & 19.54 & 223 & 53.1 & 13.25\end{array}$


100IVD/8_IN_DRY_KMAG_SN_S10772_750LB_55RPM_30SCFM_TO_COMPARE_NO_FLOW_INSERT

TIME PURG_TC2 PurgePSI PurgeCFM DEPTH(IN) DwnFrc(Ib) RPM inches/Min

$\begin{array}{llllllll}14: 58: 36: 62 & 21 & -0.6 & -5.6 & -0.03 & -0 & 48.2 & -0.82 \\ \text { 14:58:37:61 } & 21 & 20.0 & 54.9 & 0.72 & 16 & 49.9 & 4.78 \\ \text { 14:58:38:59 } & 21 & 23.7 & 54.9 & 1.53 & 20 & 49.9 & 4.78 \\ \text { 14:58:39:58 } & 21 & 24.3 & 54.7 & 2.29 & 72 & 50.6 & 13.54 \\ \text { 14:58:40:63 } & 21 & 24.5 & 54.5 & 3.09 & 52 & 51.3 & 13.54 \\ 14: 58: 41: 61 & 21 & 24.5 & 54.5 & 3.87 & 61 & 51.3 & 13.44 \\ 14: 58: 42: 60 & 21 & 24.7 & 54.4 & 4.62 & 66 & 52.1 & 13.44 \\ 14: 58: 43: 59 & 21 & 24.9 & 54.4 & 5.40 & 74 & 52.7 & 14.11 \\ 14: 58: 44: 58 & 21 & 24.9 & 54.3 & 6.12 & 79 & 52.7 & 14.11 \\ 14: 58: 45: 62 & 21 & 24.9 & 54.2 & 6.88 & 85 & 52.9 & 13.94 \\ 14: 58: 46: 61 & 21 & 25.1 & 54.2 & 7.62 & 91 & 53.0 & 13.94 \\ 14: 58: 47: 60 & 21 & 25.1 & 54.1 & 8.35 & 100 & 53.0 & 13.32 \\ 14: 58: 48: 65 & 21 & 25.1 & 54.0 & 9.10 & 108 & 53.1 & 13.32 \\ 14: 58: 49: 63 & 21 & 25.1 & 54.0 & 9.80 & 114 & 53.0 & 13.07 \\ 14: 58: 50: 62 & 21 & 25.1 & 54.0 & 10.49 & 120 & 53.0 & 13.07 \\ 14: 58: 51: 61 & 21 & 25.3 & 54.0 & 11.16 & 126 & 53.0 & 10.31 \\ 14: 58: 52: 60 & 21 & 25.3 & 53.9 & 11.84 & 129 & 53.0 & 10.31 \\ 14: 58: 53: 64 & 21 & 25.3 & 53.8 & 12.53 & 141 & 53.0 & 12.41 \\ 14: 58: 54: 63 & 21 & 25.3 & 53.8 & 13.20 & 151 & 53.0 & 12.41 \\ 14: 58: 55: 62 & 21 & 25.5 & 53.8 & 13.83 & 166 & 53.0 & 12.03 \\ 14: 58: 56: 61 & 21 & 25.5 & 53.7 & 14.43 & 183 & 53.0 & 12.03 \\ 14: 58: 57: 60 & 21 & 25.7 & 53.7 & 15.04 & 204 & 52.9 & 12.33 \\ 14: 58: 58: 59 & 21 & 25.7 & 53.6 & 15.61 & 215 & 53.0 & 12.86 \\ 14: 58: 59: 58 & 21 & 25.7 & 53.5 & 16.15 & 216 & 53.0 & 12.86 \\ 14: 59: 00: 56 & 21 & 25.7 & 53.5 & 16.69 & 221 & 53.0 & 12.99 \\ 14: 59: 01: 55 & 21 & 25.7 & 53.5 & 17.22 & 231 & 53.0 & 12.99 \\ 14: 59: 02: 54 & 21 & 25.9 & 53.5 & 17.76 & 247 & 53.0 & 13.38 \\ 14: 59: 03: 53 & 21 & 25.9 & 53.5 & 18.23 & 249 & 53.0 & 13.38 \\ 14: 59: 04: 52 & 21 & 26.1 & 53.4 & 18.73 & 244 & 53.0 & 12.81 \\ 14: 59: 05: 56 & 21 & 26.3 & 53.3 & 19.24 & 251 & 53.0 & 12.81\end{array}$


T10772.TXT

HNF-3557

Rev. 0

100IVD/8_IN_DRY_KMAG_SN_T10772_750LB_55RPM_30SCFM_TO_COMPARE_NO_FLOW_INSERT

TIME PURG_TC2 PurgePSI PurgecfM DEPTH(IN) DwnFrc(lb) RPM Inches/Min

$\begin{array}{llllllll}15: 08: 54: 75 & 21 & -0.6 & -5.5 & 0.01 & 9 & 51.7 & -0.37 \\ \text { 15:08:55:79 } & 21 & 9.3 & 54.9 & 0.86 & 31 & 52.8 & 6.01 \\ \text { 15:08:56:78 } & 22 & 25.1 & 54.2 & 1.63 & 26 & 52.8 & 6.01 \\ \text { 15:08:57:77 } & 22 & 24.9 & 54.0 & 2.38 & 22 & 52.9 & 14.00 \\ \text { 15:08:58:81 } & 21 & 24.9 & 54.0 & 3.22 & 24 & 53.0 & 14.00 \\ \text { 15:08:59:80 } & 22 & 24.7 & 54.0 & 4.00 & 24 & 53.0 & 13.70 \\ 15: 09: 00: 79 & 21 & 24.9 & 53.9 & 4.78 & 24 & 53.2 & 13.70 \\ 15: 09: 01: 78 & 21 & 24.9 & 53.9 & 5.58 & 22 & 53.3 & 14.13 \\ 15: 09: 02: 77 & 21 & 24.9 & 53.8 & 6.38 & 21 & 53.3 & 14.13 \\ 15: 09: 03: 81 & 21 & 24.9 & 53.8 & 7.19 & 26 & 53.4 & 14.31 \\ 15: 09: 04: 80 & 21 & 24.9 & 53.8 & 7.96 & 27 & 53.5 & 14.31 \\ 15: 09: 05: 79 & 21 & 25.3 & 53.8 & 8.72 & 34 & 53.5 & 13.86 \\ 15: 09: 06: 83 & 21 & 25.7 & 53.7 & 9.53 & 48 & 53.6 & 13.86 \\ 15: 09: 07: 82 & 21 & 26.1 & 53.4 & 10.28 & 57 & 53.6 & 12.82 \\ 15: 09: 08: 81 & 21 & 26.1 & 53.2 & 11.00 & 63 & 53.6 & 12.82 \\ 15: 09: 09: 80 & 21 & 26.3 & 53.1 & 11.75 & 68 & 53.6 & 12.48 \\ 15: 09: 10: 79 & 21 & 26.1 & 53.0 & 12.44 & 82 & 53.6 & 12.48 \\ 15: 09: 11: 83 & 21 & 26.3 & 52.9 & 13.20 & 90 & 53.6 & 12.37 \\ 15: 09: 12: 82 & 21 & 26.3 & 52.9 & 13.89 & 104 & 53.5 & 12.37 \\ 15: 09: 13: 81 & 21 & 26.5 & 52.8 & 14.56 & 112 & 53.4 & 12.21 \\ 15: 09: 14: 85 & 21 & 26.5 & 52.7 & 15.22 & 124 & 53.4 & 12.34 \\ 15: 09: 15: 84 & 21 & 26.5 & 52.7 & 15.89 & 136 & 53.3 & 12.34 \\ 15: 09: 16: 83 & 21 & 26.5 & 52.7 & 16.48 & 142 & 53.3 & 13.12 \\ 15: 09: 17: 82 & 21 & 26.5 & 52.7 & 17.11 & 149 & 53.3 & 13.12 \\ 15: 09: 18: 81 & 21 & 26.5 & 52.7 & 17.68 & 158 & 53.3 & 14.04 \\ 15: 09: 19: 85 & 21 & 26.6 & 52.7 & 18.27 & 171 & 53.3 & 14.04 \\ 15: 09: 20: 84 & 21 & 26.8 & 52.7 & 18.80 & 176 & 53.3 & 12.95 \\ 15: 09: 21: 83 & 21 & 26.8 & 52.6 & 19.31 & 189 & 53.3 & 12.95\end{array}$


100IVD/9_IN_DRY_KMAG_SN_Q_NFI_750LB_55RPM_3OSCFM_TO_COMPARE_STANDARD_INSERT

TIME PURG_TC2 PurgePSI PurgeCFM DEPTH(IN) DwnFrc(lb) RPM Inches/Min

$\begin{array}{llllllll}15: 20: 29: 61 & 21 & -0.6 & 14.9 & -0.00 & -0 & 54.2 & -0.25 \\ 15: 20: 30: 54 & 22 & 21.6 & 57.2 & 0.53 & -0 & 54.7 & -0.25 \\ 15: 20: 31: 59 & 22 & 22.4 & 56.8 & 1.36 & -0 & 54.4 & 4.79 \\ 15: 20: 32: 58 & 22 & 22.6 & 56.7 & 2.19 & -1 & 54.4 & 13.01 \\ 15: 20: 33: 57 & 22 & 22.6 & 56.5 & 2.94 & -0 & 53.9 & 13.01 \\ 15: 20: 34: 61 & 21 & 22.8 & 56.4 & 3.77 & -0 & 53.8 & 13.59 \\ 15: 20: 35: 60 & 21 & 22.8 & 56.4 & 4.60 & -0 & 53.8 & 13.59 \\ 15: 20: 36: 59 & 21 & 22.8 & 56.4 & 5.38 & -0 & 53.7 & 13.19 \\ 15: 20: 37: 57 & 21 & 22.8 & 56.3 & 6.20 & -0 & 53.7 & 13.19 \\ 15: 20: 38: 56 & 22 & 22.8 & 56.3 & 6.96 & -0 & 53.7 & 13.83 \\ 15: 20: 39: 61 & 21 & 22.9 & 56.2 & 7.77 & 1 & 53.7 & 13.83 \\ 15: 20: 40: 60 & 21 & 22.9 & 56.2 & 8.58 & 17 & 53.7 & 12.20 \\ 15: 20: 41: 58 & 21 & 23.1 & 56.2 & 9.33 & 32 & 53.7 & 12.20 \\ 15: 20: 42: 63 & 21 & 23.1 & 56.2 & 10.12 & 44 & 53.7 & 9.04 \\ 15: 20: 43: 62 & 21 & 23.7 & 56.0 & 10.88 & 57 & 53.8 & 9.04 \\ 15: 20: 44: 61 & 21 & 24.1 & 55.9 & 11.60 & 72 & 53.8 & 7.91 \\ 15: 20: 45: 59 & 21 & 24.3 & 55.8 & 12.34 & 89 & 53.7 & 7.91 \\ 15: 20: 46: 58 & 21 & 24.9 & 55.8 & 13.00 & 101 & 53.7 & 11.91 \\ 15: 20: 47: 63 & 21 & 25.1 & 55.6 & 13.73 & 117 & 53.7 & 11.91 \\ 15: 20: 48: 61 & 21 & 24.5 & 55.5 & 14.42 & 120 & 53.6 & 11.94 \\ 15: 20: 49: 60 & 21 & 24.9 & 55.5 & 15.04 & 127 & 53.4 & 11.94 \\ 15: 20: 50: 65 & 21 & 26.5 & 54.0 & 15.65 & 145 & 53.4 & 13.17 \\ 15: 20: 51: 64 & 21 & 27.4 & 53.4 & 16.29 & 154 & 53.3 & 13.17 \\ 15: 20: 52: 62 & 21 & 27.6 & 53.2 & 16.88 & 159 & 53.3 & 11.58 \\ 15: 20: 53: 61 & 21 & 28.0 & 53.1 & 17.48 & 167 & 53.3 & 11.58 \\ 15: 20: 54: 60 & 21 & 27.8 & 53.0 & 17.99 & 169 & 53.3 & 13.75 \\ 15: 20: 55: 65 & 21 & 25.1 & 53.0 & 18.55 & 192 & 53.3 & 11.91 \\ 15: 20: 56: 63 & 21 & 24.9 & 53.0 & 19.11 & 184 & 53.3 & 11.91\end{array}$


100IVD/9_IN_WET_UREA_SN_R_NFI_750LB_55RPM_30SCFM_TO_COMPARE_STANDARD_INSERT

TIME PURG_TC2 PurgePSI PurgeCFM DEPTH(IN) DwnFrc(lb) RPM Inches/Min

$\begin{array}{llllllll}15: 57: 10: 80 & 22 & -0.6 & 19.0 & 0.31 & -30 & 53.9 & -0.26 \\ 15: 57: 11: 79 & 22 & 23.3 & 56.2 & 1.11 & -26 & 53.9 & -0.26 \\ 15: 57: 12: 78 & 22 & 24.3 & 55.8 & 1.94 & -28 & 54.1 & 11.09 \\ 15: 57: 13: 77 & 22 & 24.7 & 55.6 & 2.78 & -26 & 53.7 & 11.09 \\ 15: 57: 14: 76 & 22 & 24.7 & 55.4 & 3.63 & -25 & 53.7 & 12.61 \\ 15: 57: 15: 75 & 22 & 24.7 & 54.8 & 4.48 & -24 & 53.7 & 12.61 \\ 15: 57: 16: 74 & 22 & 24.9 & 54.5 & 5.30 & -25 & 53.8 & 13.09 \\ 15: 57: 17: 73 & 22 & 24.9 & 54.4 & 6.16 & -24 & 53.8 & 13.09 \\ 15: 57: 18: 71 & 22 & 24.9 & 54.3 & 6.99 & -25 & 53.8 & 12.78 \\ 15: 57: 19: 70 & 22 & 24.9 & 54.2 & 7.82 & -25 & 53.9 & 12.78 \\ 15: 57: 20: 69 & 22 & 24.9 & 54.1 & 8.66 & -25 & 53.9 & 11.96 \\ 15: 57: 21: 68 & 22 & 25.1 & 54.0 & 9.50 & -21 & 53.9 & 11.96 \\ 15: 57: 22: 67 & 22 & 25.1 & 54.0 & 10.31 & -23 & 53.9 & 12.15 \\ 15: 57: 23: 66 & 22 & 25.1 & 54.0 & 11.16 & -24 & 53.9 & 12.15 \\ 15: 57: 24: 65 & 22 & 25.1 & 53.9 & 12.01 & -23 & 53.9 & 13.14 \\ 15: 57: 25: 63 & 22 & 25.1 & 53.8 & 12.84 & -24 & 53.9 & 9.89 \\ 15: 57: 26: 62 & 22 & 25.1 & 53.8 & 13.67 & -23 & 54.0 & 9.89 \\ 15: 57: 27: 61 & 22 & 25.1 & 53.8 & 14.48 & -23 & 54.0 & 13.48 \\ 15: 57: 28: 60 & 22 & 25.1 & 53.8 & 15.33 & -23 & 54.0 & 13.48 \\ 15: 57: 29: 59 & 22 & 25.1 & 53.7 & 16.17 & -23 & 54.1 & 13.78 \\ 15: 57: 30: 58 & 22 & 25.1 & 53.7 & 17.02 & -22 & 54.1 & 13.78 \\ 15: 57: 31: 57 & 22 & 25.1 & 53.7 & 17.84 & -22 & 54.1 & 12.81 \\ 15: 57: 32: 56 & 22 & 25.1 & 53.7 & 18.68 & -21 & 54.1 & 12.81 \\ 15: 57: 33: 54 & 22 & 25.3 & 53.7 & 19.49 & -22 & 54.1 & 13.47 \\ 15: 57: 34: 53 & 22 & 25.3 & 53.7 & 20.31 & -20 & 54.1 & 13.47\end{array}$


100IVD/9_IN_WET_UREA_SN_S_NFI_750LB_55RPM_30SCFM_TO_COMPARE_STANDARD_INSERT

TIME PURG_TC2 PurgePSI PurgeCFM DEPTH(IN) DwnFrc(Ib) RPM Inches/Min

$\begin{array}{llllllll}16: 39: 10: 07 & 21 & 8.5 & 38.2 & 0.20 & -31 & 56.6 & 0.09 \\ 16: 39: 11: 06 & 21 & 24.1 & 55.8 & 0.97 & -31 & 56.5 & 0.09 \\ 16: 39: 12: 05 & 21 & 24.7 & 54.8 & 1.78 & -31 & 55.8 & 7.89 \\ \text { 16:39:13:04 } & 21 & 25.1 & 54.4 & 2.59 & -30 & 55.8 & 12.93 \\ 16: 39: 14: 03 & 21 & 25.1 & 54.2 & 3.40 & -28 & 55.4 & 12.93 \\ 16: 39: 15: 02 & 21 & 25.1 & 54.0 & 4.21 & -25 & 55.4 & 12.42 \\ 16: 39: 16: 00 & 21 & 25.3 & 54.0 & 5.02 & -20 & 55.3 & 12.42 \\ 16: 39: 16: 99 & 21 & 25.3 & 54.0 & 5.83 & -19 & 55.3 & 12.27 \\ 16: 39: 17: 98 & 21 & 25.3 & 53.9 & 6.63 & -17 & 55.3 & 12.27 \\ 16: 39: 18: 97 & 21 & 25.3 & 53.8 & 7.42 & -17 & 55.2 & 12.93 \\ 16: 39: 19: 96 & 21 & 25.3 & 53.8 & 8.22 & -18 & 55.0 & 12.93 \\ 16: 39: 20: 95 & 21 & 25.3 & 53.7 & 9.01 & -17 & 55.0 & 12.42 \\ 16: 39: 21: 94 & 21 & 25.3 & 53.7 & 9.82 & -18 & 54.9 & 12.42 \\ 16: 39: 22: 92 & 21 & 25.1 & 53.7 & 10.65 & -20 & 54.8 & 12.53 \\ 16: 39: 23: 91 & 21 & 25.1 & 53.6 & 11: 44 & -18 & 54.8 & 12.53 \\ 16: 39: 24: 90 & 21 & 25.1 & 53.6 & 12.25 & -17 & 54.9 & 10.67 \\ 16: 39: 25: 89 & 21 & 25.1 & 53.6 & 13.07 & -17 & 55.0 & 10.67 \\ 16: 39: 26: 88 & 21 & 25.3 & 53.6 & 13.82 & -18 & 55.0 & 10.88 \\ 16: 39: 27: 87 & 21 & 25.3 & 53.6 & 14.64 & -18 & 54.9 & 10.88 \\ 16: 39: 28: 86 & 21 & 25.3 & 53.6 & 15.43 & -18 & 55.0 & 12.83 \\ 16: 39: 29: 85 & 21 & 25.3 & 53.5 & 16.23 & -21 & 55.0 & 12.83 \\ 16: 39: 30: 83 & 21 & 25.3 & 53.5 & 17.07 & -20 & 55.1 & 14.45 \\ 16: 39: 31: 82 & 21 & 25.3 & 53.5 & 17.84 & -20 & 55.1 & 14.45 \\ 16: 39: 32: 81 & 21 & 25.3 & 53.5 & 18.67 & -20 & 55.1 & 13.15 \\ 16: 39: 33: 80 & 21 & 25.3 & 53.5 & 19.43 & -18 & 55.1 & 13.15\end{array}$


T_NFI.TXT

HNF-3557

Rev. 0

100IVD/9_IN_WET_UREA_SN_T_NFI_750LB_55RPM_30SCFM_TO_COMPARE_STANDARD_INSERT

TIME PURG_TC2 PurgePSI PurgeCFM DEPTH(IN) DwnFrc(lb) RPM inches/Min

$\begin{array}{lllllllc}16: 47: 30: 61 & 21 & 2.5 & 23.0 & -0.00 & -31 & 51.5 & -0.32 \\ 16: 47: 31: 60 & 21 & 12.2 & 54.7 & 0.27 & -31 & 53.8 & -0.32 \\ 16: 47: 32: 59 & 21 & 22.8 & 54.8 & 1.08 & -31 & 53.8 & 5.28 \\ 16: 47: 33: 57 & 21 & 23.1 & 54.8 & 1.90 & -31 & 54.3 & 5.28 \\ 16: 47: 34: 56 & 21 & 23.1 & 54.8 & 2.74 & -28 & 54.3 & 12.62 \\ 16: 47: 35: 55 & 21 & 23.1 & 54.8 & 3.54 & -17 & 54.3 & 12.62 \\ 16: 47: 36: 54 & 21 & 23.1 & 54.8 & 4.35 & -17 & 54.6 & 12.18 \\ 16: 47: 37: 53 & 21 & 23.1 & 54.8 & 5.16 & -17 & 54.7 & 12.18 \\ 16: 47: 38: 52 & 21 & 23.1 & 54.8 & 5.99 & -16 & 54.7 & 12.64 \\ 16: 47: 39: 51 & 21 & 23.3 & 54.8 & 6.82 & -14 & 54.6 & 12.64 \\ 16: 47: 40: 49 & 21 & 23.3 & 54.8 & 7.61 & -6 & 54.6 & 12.83 \\ 16: 47: 41: 48 & 21 & 23.3 & 54.8 & 8.47 & -7 & 54.7 & 12.83 \\ 16: 47: 42: 47 & 21 & 23.3 & 54.8 & 9.28 & -6 & 54.7 & 12.09 \\ 16: 47: 43: 46 & 21 & 23.3 & 54.8 & 10.11 & -10 & 54.7 & 12.09 \\ 16: 47: 44: 45 & 21 & 23.3 & 54.8 & 10.94 & -15 & 54.7 & 13.39 \\ 16: 47: 45: 44 & 21 & 23.5 & 54.8 & 11.74 & -10 & 54.8 & 13.39 \\ 16: 47: 46: 43 & 21 & 23.5 & 54.8 & 12.53 & -15 & 54.8 & 11.10 \\ 16: 47: 47: 42 & 21 & 23.3 & 54.8 & 13.34 & -8 & 55.0 & 11.10 \\ 16: 47: 48: 40 & 21 & 23.5 & 54.8 & 14.11 & -23 & 55.1 & 11.45 \\ 16: 47: 49: 39 & 21 & 23.5 & 54.8 & 14.89 & -25 & 55.1 & 11.45 \\ 16: 47: 50: 38 & 21 & 23.5 & 54.8 & 15.73 & -27 & 55.0 & 13.57 \\ 16: 47: 51: 37 & 21 & 23.5 & 54.8 & 16.55 & -26 & 55.0 & 12.50 \\ 16: 47: 52: 36 & 21 & 23.5 & 54.8 & 17.39 & -26 & 55.0 & 12.50 \\ 16: 47: 53: 35 & 21 & 23.5 & 54.8 & 18.17 & -22 & 55.0 & 14.19 \\ 16: 47: 54: 34 & 21 & 23.5 & 54.8 & 19.01 & -25 & 55.0 & 14.19 \\ 16: 47: 55: 32 & 21 & 23.5 & 54.8 & 19.79 & -26 & 55.0 & 14.21 \\ 16: 47: 56: 31 & 21 & 23.5 & 54.8 & 20.62 & -15 & 54.9 & 14.21 \\ 16: 47: 57: 30 & 21 & 23.5 & 54.8 & 21.41 & -13 & 54.9 & 13.53 \\ 16: 47: 58: 29 & 21 & 23.5 & 54.8 & 22.18 & -13 & 54.9 & 13.53 \\ 16: 47: 59: 28 & 21 & 23.5 & 54.8 & 22.99 & -14 & 55.0 & 14.54 \\ 16: 48: 00: 27 & 21 & 23.7 & 54.8 & 23.28 & 463 & 55.0 & 14.54 \\ 16: 48: 01: 26 & 21 & 24.1 & 54.7 & 23.33 & 546 & 55.0 & 7.00 \\ 16: 48: 02: 25 & 21 & 24.7 & 54.5 & 23.36 & 640 & 55.0 & 7.00 \\ 16: 48: 03: 23 & 21 & 24.7 & 54.4 & 22.98 & 674 & 55.2 & 0.12\end{array}$


100IVD/9_IN_WET_UREA_SN_U_NFI_750LB_55RPM_30SCFM_TO_COMPARE_STANDARD_INSERT

TIME PURG_TC2 PurgePSI PurgeCFM DEPTH(IN) DwnFrc(Ib) RPM Inches/Min

$\begin{array}{lccccccc}14: 18: 08: 80 & 21 & -0.8 & -5.7 & 0.00 & 7 & 53.6 & -0.79 \\ 14: 18: 09: 73 & 21 & 9.3 & 53.2 & 0.79 & 5 & 53.6 & -0.76 \\ 14: 18: 10: 72 & 21 & 22.6 & 53.2 & 1.59 & 8 & 54.3 & -0.76 \\ 14: 18: 11: 71 & 21 & 23.7 & 53.3 & 2.42 & 8 & 54.2 & 12.70 \\ 14: 18: 12: 70 & 21 & 24.1 & 53.3 & 3.24 & 8 & 54.2 & 12.22 \\ 14: 18: 13: 69 & 21 & 24.3 & 53.3 & 4.08 & 9 & 54.3 & 12.22 \\ 14: 18: 14: 68 & 21 & 24.3 & 53.3 & 4.87 & 9 & 54.4 & 13.06 \\ 14: 18: 15: 67 & 21 & 24.3 & 53.3 & 5.73 & 9 & 54.4 & 13.06 \\ 14: 18: 16: 65 & 21 & 24.3 & 53.3 & 6.54 & 9 & 54.6 & 12.79 \\ 14: 18: 17: 64 & 21 & 24.5 & 53.3 & 7.38 & 9 & 54.7 & 12.79 \\ 14: 18: 18: 63 & 21 & 24.5 & 53.2 & 8.20 & 10 & 54.7 & 12.65 \\ 14: 18: 19: 62 & 21 & 24.5 & 53.2 & 9.03 & 9 & 54.7 & 12.65 \\ 14: 18: 20: 61 & 21 & 24.5 & 53.1 & 9.88 & 10 & 54.7 & 10.40 \\ 14: 18: 21: 60 & 21 & 24.7 & 53.1 & 10.70 & 9 & 54.7 & 10.40 \\ 14: 18: 22: 59 & 21 & 24.7 & 53.0 & 11.53 & 10 & 54.8 & 10.55 \\ 14: 18: 23: 58 & 21 & 24.5 & 53.0 & 12.36 & 9 & 54.8 & 10.55 \\ 14: 18: 24: 56 & 21 & 24.5 & 53.0 & 13.21 & 10 & 54.8 & 12.72 \\ 14: 18: 25: 55 & 21 & 24.7 & 52.9 & 14.03 & 11 & 54.9 & 12.72 \\ 14: 18: 26: 54 & 21 & 24.5 & 52.9 & 14.84 & 10 & 54.9 & 14.37 \\ 14: 18: 27: 53 & 21 & 24.7 & 52.9 & 15.68 & 10 & 54.9 & 14.37 \\ 14: 18: 28: 52 & 21 & 24.7 & 52.9 & 16.50 & 10 & 54.9 & 13.94 \\ 14: 18: 29: 51 & 21 & 24.7 & 52.9 & 17.33 & 12 & 54.9 & 13.94 \\ 14: 18: 30: 50 & 21 & 24.7 & 52.9 & 18.16 & 11 & 54.9 & 13.94 \\ 14: 18: 31: 48 & 21 & 24.7 & 52.9 & 18.95 & 12 & 54.8 & 13.94 \\ 14: 18: 32: 47 & 21 & 24.7 & 52.9 & 19.75 & 14 & 54.8 & 14.61\end{array}$


U10772.TXT

HNF-3557

Rev. 0

100IVD/8_IN_WET_UREA_SN_U10772_750LB_55RPM_30SCFM_TO_COMPARE_NO_FLOW_INSERT

TIME PURG_TC2 PurgePSI PurgeCFM DEPTH(IN) DwnFrc(lb) RPM Inches/Min

$\begin{array}{llllllll}14: 32: 46: 51 & 21 & -0.8 & -5.7 & 0.04 & -8 & 55.6 & -0.32 \\ 14: 32: 47: 50 & 22 & 2.5 & 55.9 & 0.75 & -7 & 55.6 & -0.32 \\ 14: 32: 48: 49 & 22 & 24.7 & 54.2 & 1.49 & -4 & 55.6 & 9.43 \\ 14: 32: 49: 53 & 22 & 24.9 & 53.8 & 2.33 & -3 & 54.9 & 9.43 \\ 14: 32: 50: 52 & 22 & 24.9 & 53.6 & 3.11 & -2 & 54.7 & 12.41 \\ 14: 32: 51: 51 & 22 & 24.7 & 53.6 & 3.89 & -3 & 54.7 & 12.41 \\ 14: 32: 52: 50 & 21 & 24.7 & 53.5 & 4.74 & -3 & 54.7 & 12.56 \\ 14: 32: 53: 49 & 22 & 24.7 & 53.5 & 5.51 & -3 & 54.6 & 12.56 \\ 14: 32: 54: 53 & 21 & 24.7 & 53.5 & 6.35 & -3 & 54.6 & 12.64 \\ 14: 32: 55: 52 & 21 & 24.7 & 53.4 & 7.17 & -2 & 54.6 & 12.64 \\ 14: 32: 56: 51 & 22 & 24.7 & 53.4 & 7.91 & -3 & 54.7 & 13.50 \\ 14: 32: 57: 55 & 22 & 24.7 & 53.4 & 8.72 & -2 & 54.7 & 13.50 \\ 14: 32: 58: 54 & 22 & 24.7 & 53.4 & 9.55 & -2 & 54.6 & 11.60 \\ 14: 32: 59: 53 & 21 & 24.7 & 53.4 & 10.33 & -2 & 54.6 & 11.60 \\ 14: 33: 00: 52 & 21 & 24.7 & 53.4 & 11.16 & -2 & 54.6 & 12.28 \\ 14: 33: 01: 50 & 21 & 24.7 & 53.4 & 11.92 & -1 & 54.6 & 12.28 \\ 14: 33: 02: 55 & 21 & 24.7 & 53.4 & 12.75 & -2 & 54.7 & 10.89 \\ 14: 33: 03: 54 & 21 & 24.7 & 53.4 & 13.60 & 1 & 54.7 & 14.26 \\ 14: 33: 04: 53 & 21 & 24.7 & 53.3 & 14.41 & 4 & 54.7 & 14.26 \\ 14: 33: 05: 57 & 21 & 24.7 & 53.3 & 15.26 & 6 & 54.7 & 12.69 \\ 14: 33: 06: 56 & 21 & 24.7 & 53.3 & 16.09 & 10 & 54.7 & 12.69 \\ 14: 33: 07: 55 & 21 & 24.7 & 53.3 & 16.89 & 11 & 54.7 & 14.11 \\ 14: 33: 08: 53 & 21 & 24.7 & 53.2 & 17.76 & 10 & 54.7 & 14.11 \\ 14: 33: 09: 52 & 21 & 24.7 & 53.2 & 18.54 & 10 & 54.7 & 13.94 \\ 14: 33: 10: 57 & 22 & 24.7 & 53.1 & 19.37 & 8 & 54.6 & 13.94\end{array}$


.100IVD/8_IN_WET_UREA_SN_V10772_750LB_55RPM_30SCFM_TO_COMPARE_NO_FLOW_INSERT

TIME PURG_TC2 PurgePSI PurgeCFM DEPTH(IN) DwnFrc(lb) RPM Inches/Min

$\begin{array}{lccccccc}14: 46: 00: 35 & 22 & 3.7 & 38.0 & -0.05 & -8 & 55.6 & 0.01 \\ 14: 46: 01: 28 & 22 & 19.4 & 53.3 & 0.77 & -8 & 55.5 & 4.02 \\ 14: 46: 02: 27 & 22 & 23.9 & 53.3 & 1.58 & -6 & 55.5 & 4.02 \\ 14: 46: 03: 26 & 22 & 24.1 & 53.3 & 2.41 & -6 & 54.8 & 12.12 \\ 14: 46: 04: 25 & 22 & 23.9 & 53.3 & 3.23 & -4 & 54.5 & 12.12 \\ 14: 46: 05: 24 & 22 & 23.9 & 53.3 & 4.03 & 9 & 54.5 & 12.72 \\ 14: 46: 06: 22 & 22 & 23.7 & 53.3 & 4.85 & -1 & 54.6 & 12.72 \\ 14: 46: 07: 21 & 22 & 23.7 & 53.2 & 5.67 & 1 & 54.6 & 12.42 \\ 14: 46: 08: 20 & 22 & 23.9 & 53.2 & 6.50 & 0 & 54.6 & 12.42 \\ 14: 46: 09: 19 & 22 & 23.9 & 53.2 & 7.33 & 7 & 54.7 & 13.70 \\ 14: 46: 10: 18 & 22 & 23.9 & 53.1 & 8.16 & 5 & 54.7 & 13.70 \\ 14: 46: 11: 17 & 22 & 23.9 & 53.1 & 8.98 & 6 & 54.7 & 11.59 \\ 14: 46: 12: 16 & 22 & 24.1 & 53.0 & 9.81 & 5 & 54.6 & 11.59 \\ 14: 46: 13: 15 & 22 & 23.9 & 52.9 & 10.58 & 5 & 54.6 & 10.65 \\ 14: 46: 14: 13 & 22 & 23.9 & 52.9 & 11.44 & 4 & 54.6 & 10.65 \\ 14: 46: 15: 12 & 22 & 24.1 & 52.9 & 12.24 & 7 & 54.5 & 11.10 \\ 14: 46: 16: 11 & 22 & 23.9 & 52.9 & 13.03 & 7 & 54.5 & 11.10 \\ 14: 46: 17: 10 & 22 & 24.1 & 52.8 & 13.90 & 5 & 54.5 & 13.46 \\ 14: 46: 18: 09 & 22 & 23.9 & 52.8 & 14.70 & 8 & 54.7 & 13.46 \\ 14: 46: 19: 08 & 22 & 24.1 & 52.8 & 15.53 & 9 & 54.8 & 15.18 \\ 14: 46: 20: 07 & 22 & 24.1 & 52.8 & 16.35 & 8 & 54.8 & 15.18 \\ 14: 46: 21: 05 & 22 & 24.3 & 52.8 & 17.15 & 8 & 55.0 & 13.19 \\ 14: 46: 22: 04 & 22 & 24.3 & 52.7 & 17.99 & 9 & 55.0 & 13.19 \\ 14: 46: 23: 03 & 22 & 24.3 & 52.7 & 18.79 & 7 & 55.0 & 15.09 \\ 14: 46: 24: 02 & 22 & 24.3 & 52.7 & 19.61 & 5 & 55.0 & 15.09\end{array}$


100IVD/8_IN_WET_SAND_SN_W10772_750LB_55RPM_30SCFM_TO_COMPARE_NO_FLOW_INSERT

TIME PURG_TC2 PurgePSI PurgecFM DEPTH(IN) DwnFrc(lb) RPM Inches/Min

\begin{tabular}{|c|c|c|c|c|c|c|c|}
\hline $15: 08: 47: 28$ & 21 & -0.6 & -5.4 & 0.01 & 7 & 55.1 & -0.70 \\
\hline $15: 08: 48: 21$ & 22 & 18.1 & 54.8 & 0.60 & 7 & 55.3 & 0.83 \\
\hline $15: 08: 49: 20$ & 22 & 22.6 & 54.5 & 1.41 & 8 & 55.0 & 0.83 \\
\hline $15: 08: 50: 19$ & 22 & 22.6 & 54.4 & 2.21 & 6 & 55.0 & 12.87 \\
\hline $15: 08: 51: 18$ & 22 & 22.6 & 54.2 & 3.03 & 6 & 54.6 & 12.87 \\
\hline $15: 08: 52: 17$ & 22 & 22.6 & 54.1 & 3.85 & 6 & 54.6 & 13.12 \\
\hline $15: 08: 53: 16$ & 22 & 22.8 & 54.0 & 4.66 & 11 & 54.5 & 13.12 \\
\hline $15: 08: 54: 14$ & 22 & 22.6 & 54.0 & 5.48 & -1 & 54.4 & 12.89 \\
\hline $15: 08: 55: 13$ & 22 & 22.4 & 53.9 & 6.29 & -1 & 54.4 & 12.89 \\
\hline $08: 56: 12$ & 22 & 22.4 & 53.8 & 7.11 & 3 & 54.5 & 13.00 \\
\hline$: 08: 57: 11$ & 22 & 22.4 & 53.8 & 7.95 & 0 & 54.5 & 13.00 \\
\hline$: 08: 58: 10$ & 22 & 22.4 & 53.8 & 8.77 & 0 & 54.5 & 13.14 \\
\hline $08: 59: 09$ & 22 & 22.6 & 53.8 & 9.56 & -1 & 54.5 & 13.14 \\
\hline $09: 00: 08$ & 22 & 22.6 & 53.8 & 10.39 & -1 & 54.6 & 12.83 \\
\hline 09:01:06 & 22 & 22.6 & 53.8 & 11.22 & -1 & 54.6 & 12.83 \\
\hline $09: 02: 05$ & 22 & 22.4 & 53.8 & 12.03 & -1 & 54.7 & 10.97 \\
\hline 09:03:04 & 22 & 22.4 & 53.8 & 12.85 & -1 & 54.7 & 10.97 \\
\hline $09: 04: 03$ & 22 & 22.4 & 53.8 & 13.68 & -1 & 54.7 & 10.76 \\
\hline 09:05:02 & 22 & 22.4 & 53.8 & 14.47 & 0 & 54.7 & 10.76 \\
\hline $09: 06: 01$ & 22 & 22.4 & 53.8 & 15.31 & 1 & 54.7 & 14.54 \\
\hline $99: 07: 00$ & 22 & 22.4 & 53.7 & 16.15 & 7 & 54.7 & 14.54 \\
\hline $9: 07: 99$ & 22 & 22.6 & 53.7 & 16.94 & 13 & 54.8 & 13.81 \\
\hline$: 08: 97$ & 22 & 22.6 & 53.7 & 17.77 & 13 & 54.8 & 14.20 \\
\hline$: 09: 96$ & 22 & 22.6 & 53.6 & 18.52 & 38 & 54.8 & 14.20 \\
\hline $9: 10: 95$ & 21 & 22.8 & 53.6 & 19.33 & 28 & 54.8 & 15.95 \\
\hline
\end{tabular}


100IVD/8_IN_WET_SAND_SN_X10772_750LB_55RPM_30SCFM_TO_COMPARE_NO_FLOW_INSERT

TIME PURG_TC2 PurgePSI PurgeCFM DEPTH(IN) DwnFrc(lb) RPM Inches/Min

$\begin{array}{lccccccc}15: 21: 03: 88 & 22 & -0.8 & -5.7 & -0.02 & -4 & 55.9 & -0.96 \\ 15: 21: 04: 82 & 22 & 2.3 & 52.5 & 0.61 & -4 & 55.9 & 3.42 \\ 15: 21: 05: 81 & 22 & 22.8 & 53.5 & 1.42 & 2 & 55.6 & 3.42 \\ 15: 21: 06: 80 & 22 & 23.1 & 53.5 & 2.27 & 10 & 55.6 & 13.42 \\ 15: 21: 07: 78 & 22 & 23.1 & 53.5 & 3.07 & 9 & 55.0 & 13.42 \\ 15: 21: 08: 77 & 22 & 23.1 & 53.5 & 3.85 & 22 & 54.9 & 13.55 \\ 15: 21: 09: 76 & 22 & 23.1 & 53.5 & 4.66 & 23 & 54.9 & 13.55 \\ 15: 21: 10: 75 & 22 & 22.9 & 53.5 & 5.49 & 15 & 54.8 & 13.74 \\ 15: 21: 11: 74 & 22 & 23.1 & 53.5 & 6.27 & 22 & 54.8 & 13.74 \\ 15: 21: 12: 73 & 22 & 23.1 & 53.5 & 7.09 & 20 & 54.8 & 13.41 \\ 15: 21: 13: 72 & 22 & 22.9 & 53.6 & 7.93 & 27 & 54.8 & 13.41 \\ 15: 21: 14: 70 & 22 & 23.1 & 53.5 & 8.68 & 27 & 54.8 & 13.40 \\ 15: 21: 15: 69 & 22 & 22.9 & 53.6 & 9.47 & 36 & 54.8 & 13.40 \\ 15: 21: 16: 68 & 22 & 22.9 & 53.6 & 10.27 & 46 & 54.8 & 11.45 \\ 15: 21: 17: 67 & 22 & 23.1 & 53.5 & 11.01 & 53 & 54.9 & 11.45 \\ 15: 21: 18: 66 & 22 & 23.1 & 53.5 & 11.78 & 51 & 54.9 & 11.21 \\ 15: 21: 19: 65 & 22 & 23.1 & 53.5 & 12.54 & 38 & 54.9 & 11.21 \\ 15: 21: 20: 64 & 22 & 23.1 & 53.5 & 13.35 & 45 & 54.9 & 12.60 \\ 15: 21: 21: 63 & 22 & 23.1 & 53.5 & 14.16 & 43 & 54.9 & 12.60 \\ 15: 21: 22: 61 & 22 & 23.1 & 53.5 & 14.95 & 37 & 54.8 & 14.31 \\ 15: 21: 23: 60 & 22 & 23.1 & 53.5 & 15.69 & 36 & 54.7 & 14.31 \\ 15: 21: 24: 59 & 22 & 23.3 & 53.5 & 16.50 & 33 & 54.7 & 13.68 \\ 15: 21: 25: 58 & 22 & 23.3 & 53.5 & 17.23 & 33 & 54.6 & 13.68 \\ 15: 21: 26: 57 & 22 & 23.1 & 53.5 & 18.03 & 51 & 54.5 & 13.87 \\ 15: 21: 27: 56 & 22 & 23.3 & 53.5 & 18.80 & 49 & 54.5 & 13.87 \\ 15: 21: 28: 55 & 22 & 23.3 & 53.5 & 19.48 & 38 & 54.7 & 16.45 \\ 15: 21: 28: 77 & 22 & 23.3 & 47.5 & 19.49 & 38 & 54.7 & 16.45 \\ 15: 21: 29: 70 & 22 & 1.3 & -5.3 & 19.48 & -2 & 54.9 & 7.44 \\ 15: 21: 30: 69 & 22 & -0.6 & -5.4 & 19.49 & -6 & 54.9 & 7.44\end{array}$


Y10772.TXT

HNF-3557

Rev. 0

100IVD/8_IN_WET_SAND_SN_Y10772_750LB_55RPM_30SCFM_TO_COMPARE_NO_FLOW_INSERT

TIME PURG_TC2 PurgePSI PurgeCFM DEPTH(IN) DwnFrc(lb) RPM Inches/Min

$\begin{array}{llllllll}16: 22: 50: 04 & 21 & -0.8 & -5.5 & 0.09 & -7 & 52.9 & -0.80 \\ 16: 22: 50: 97 & 22 & 20.0 & 52.9 & 0.85 & 10 & 54.0 & -0.80 \\ 16: 22: 51: 96 & 22 & 24.5 & 52.8 & 1.65 & 8 & 54.1 & 13.12 \\ 16: 22: 52: 95 & 22 & 24.3 & 52.8 & 2.47 & 12 & 54.1 & 13.12 \\ 16: 22: 53: 94 & 22 & 24.3 & 52.8 & 3.27 & 16 & 54.1 & 13.23 \\ 16: 22: 54: 93 & 22 & 24.3 & 52.7 & 4.09 & 18 & 54.3 & 13.23 \\ 16: 22: 55: 91 & 22 & 24.1 & 52.8 & 4.84 & 18 & 54.3 & 14.01 \\ 16: 22: 56: 90 & 21 & 24.3 & 52.7 & 5.67 & 21 & 54.4 & 14.01 \\ 16: 22: 57: 89 & 22 & 24.3 & 52.7 & 6.46 & 20 & 54.5 & 13.39 \\ 16: 22: 58: 88 & 21 & 24.3 & 52.7 & 7.26 & 15 & 54.5 & 13.39 \\ 16: 22: 59: 87 & 21 & 24.3 & 52.7 & 8.07 & 20 & 54.6 & 14.48 \\ 16: 23: 00: 86 & 21 & 24.3 & 52.7 & 8.85 & 16 & 54.6 & 14.48 \\ 16: 23: 01: 85 & 21 & 24.3 & 52.7 & 9.66 & 15 & 54.6 & 12.06 \\ 16: 23: 02: 83 & 22 & 24.5 & 52.7 & 10.46 & 14 & 54.6 & 12.06 \\ 16: 23: 03: 82 & 22 & 24.5 & 52.7 & 11.23 & 18 & 54.6 & 10.30 \\ 16: 23: 04: 81 & 22 & 24.5 & 52.6 & 12.06 & 15 & 54.7 & 10.30 \\ 16: 23: 05: 80 & 21 & 24.3 & 52.6 & 12.81 & 16 & 54.8 & 12.56 \\ 16: 23: 06: 79 & 21 & 24.5 & 52.6 & 13.64 & 12 & 54.8 & 12.56 \\ 16: 23: 07: 78 & 22 & 24.7 & 52.6 & 14.42 & 16 & 54.8 & 13.92 \\ 16: 23: 08: 77 & 22 & 24.7 & 52.6 & 15.22 & 13 & 54.8 & 13.92 \\ 16: 23: 09: 76 & 21 & 24.9 & 52.5 & 16.01 & 12 & 54.8 & 15.00 \\ 16: 23: 10: 74 & 21 & 24.3 & 52.5 & 16.83 & 14 & 54.8 & 15.00 \\ 16: 23: 11: 73 & 22 & 24.3 & 52.5 & 17.58 & 18 & 54.7 & 14.27 \\ 16: 23: 12: 72 & 22 & 24.3 & 52.5 & 18.38 & 20 & 54.7 & 14.27 \\ 16: 23: 13: 71 & 22 & 24.5 & 52.5 & 19.17 & 18 & 54.7 & 15.53 \\ 16: 23: 14: 70 & 22 & 24.1 & 50.7 & 19.56 & 18 & 54.7 & 15.53\end{array}$


100IVD/9_IN_WET_SAND_SN_V_NFI_750LB_55RPM_3OSCFM_TO_COMPARE_STANDARD_INSERT

TIME PURG_TC2 PurgePSI PurgeCFM DEPTH(IN) DwnFrc(Ib) RPM Inches/Min

$\begin{array}{lllllllc}16: 47: 18: 09 & 21 & -0.6 & -5.5 & 0.07 & -3 & 56.0 & -0.71 \\ 16: 47: 19: 02 & 22 & 16.1 & 53.8 & 0.86 & 2 & 55.9 & 3.94 \\ 16: 47: 20: 01 & 22 & 21.6 & 53.8 & 1.68 & 1 & 55.9 & 3.94 \\ 16: 47: 21: 00 & 22 & 21.6 & 53.8 & 2.51 & 1 & 55.2 & 13.21 \\ 16: 47: 21: 99 & 22 & 21.6 & 53.8 & 3.35 & 2 & 54.9 & 13.21 \\ 16: 47: 22: 97 & 22 & 21.6 & 53.8 & 4.15 & 1 & 54.9 & 13.36 \\ 16: 47: 23: 96 & 22 & 21.8 & 53.9 & 4.97 & 3 & 54.9 & 13.36 \\ 16: 47: 24: 95 & 22 & 21.6 & 53.9 & 5.79 & 5 & 55.0 & 13.48 \\ 16: 47: 25: 94 & 22 & 21.8 & 53.9 & 6.63 & 8 & 55.0 & 13.48 \\ 16: 47: 26: 93 & 22 & 22.0 & 53.9 & 7.42 & 11 & 55.0 & 13.88 \\ 16: 47: 27: 92 & 22 & 22.0 & 53.9 & 8.23 & 12 & 55.1 & 13.88 \\ 16: 47: 28: 91 & 21 & 22.4 & 53.9 & 9.01 & 18 & 55.1 & 13.76 \\ 16: 47: 29: 89 & 22 & 22.6 & 53.9 & 9.80 & 25 & 55.1 & 13.76 \\ 16: 47: 30: 88 & 21 & 23.3 & 53.9 & 10.58 & 41 & 55.1 & 10.43 \\ 16: 47: 31: 87 & 22 & 24.1 & 53.8 & 11.32 & 50 & 55.1 & 10.43 \\ 16: 47: 32: 86 & 22 & 24.7 & 52.9 & 12.10 & 56 & 54.9 & 11.67 \\ 16: 47: 33: 85 & 22 & 24.7 & 52.8 & 12.84 & 57 & 54.8 & 11.67 \\ 16: 47: 34: 84 & 22 & 25.1 & 52.7 & 13.60 & 66 & 54.8 & 12.99 \\ 16: 47: 35: 83 & 21 & 25.3 & 52.6 & 14.34 & 70 & 54.8 & 12.99 \\ 16: 47: 36: 81 & 22 & 25.3 & 52.5 & 15.12 & 66 & 54.8 & 14.66 \\ 16: 47: 37: 80 & 22 & 22.4 & 52.5 & 15.93 & 6 & 54.8 & 14.66 \\ 16: 47: 38: 79 & 22 & 22.4 & 52.6 & 16.71 & 30 & 54.8 & 13.40 \\ 16: 47: 39: 78 & 22 & 22.0 & 52.7 & 17.53 & 41 & 54.7 & 13.40 \\ 16: 47: 40: 77 & 22 & 22.0 & 52.8 & 18.32 & 40 & 54.7 & 14.37 \\ 16: 47: 41: 76 & 22 & 22.0 & 52.8 & 19.10 & 41 & 54.6 & 15.68\end{array}$


100IVD/9_IN_WET_SAND_SN_W_NFI_750LB_55RPM_30SCFM_TO_COMPARE_STANDARD_INSERT

TIME PURG_TC2 PurgePSI PurgeCFM DEPTH(IN) DwnFrc(Ib) RPM inches/Min

$\begin{array}{lccccccc}16: 58: 01: 92 & 22 & -0.6 & -5.6 & 0.15 & -5 & 54.1 & 0.34 \\ 16: 58: 02: 91 & 22 & 8.1 & 1.9 & 0.91 & 13 & 55.1 & 0.34 \\ 16: 58: 03: 90 & 22 & 22.0 & 1.9 & 1.77 & 0 & 54.9 & 13.48 \\ 16: 58: 04: 89 & 22 & 21.6 & 1.9 & 2.57 & -1 & 54.9 & 13.48 \\ 16: 58: 05: 88 & 22 & 21.6 & 1.9 & 3.41 & 1 & 54.7 & 13.28 \\ 16: 58: 06: 87 & 22 & 21.8 & 1.7 & 4.24 & 0 & 54.8 & 13.28 \\ 16: 58: 07: 85 & 22 & 21.8 & 1.7 & 5.05 & 3 & 54.8 & 14.14 \\ 16: 58: 08: 84 & 22 & 21.8 & 1.7 & 5.86 & 6 & 54.9 & 14.14 \\ 16: 58: 09: 83 & 22 & 21.8 & 1.7 & 6.68 & 13 & 55.0 & 13.99 \\ 16: 58: 10: 82 & 22 & 21.8 & 1.6 & 7.48 & 18 & 55.0 & 13.99 \\ 16: 58: 11: 81 & 22 & 21.8 & 1.5 & 8.27 & 18 & 55.0 & 14.78 \\ 16: 58: 12: 80 & 22 & 22.0 & 1.5 & 9.09 & 21 & 55.1 & 14.78 \\ 16: 58: 13: 79 & 22 & 22.6 & 1.4 & 9.89 & 30 & 55.1 & 11.71 \\ 16: 58: 14: 77 & 22 & 23.7 & 1.4 & 10.64 & 48 & 55.0 & 11.71 \\ 16: 58: 15: 76 & 22 & 25.3 & 1.4 & 11.42 & 58 & 55.0 & 9.18 \\ 16: 58: 16: 75 & 22 & 22.2 & 1.4 & 12.24 & 0 & 55.0 & 9.18 \\ 16: 58: 17: 74 & 22 & 21.8 & 1.4 & 13.05 & 3 & 55.1 & 11.06 \\ 16: 58: 18: 73 & 22 & 22.2 & 1.4 & 13.86 & 13 & 55.1 & 11.06 \\ 16: 58: 19: 72 & 22 & 22.6 & 1.4 & 14.68 & 11 & 55.0 & 14.56 \\ 16: 58: 20: 71 & 22 & 22.0 & 1.4 & 15.46 & 4 & 54.9 & 14.56 \\ 16: 58: 21: 70 & 22 & 21.8 & 1.4 & 16.27 & 2 & 54.9 & 15.13 \\ 16: 58: 22: 68 & 22 & 21.8 & 1.4 & 17.06 & 2 & 54.9 & 15.13 \\ 16: 58: 23: 67 & 22 & 22.0 & 1.4 & 17.86 & 2 & 55.0 & 14.39 \\ 16: 58: 24: 66 & 22 & 22.0 & 1.4 & 18.69 & 3 & 55.0 & 14.39 \\ 16: 58: 25: 65 & 22 & 22.0 & 1.4 & 19.42 & 4 & 55.1 & 16.52\end{array}$


100IVD/9_IN_WET_SAND_SN_X_NFI_750LB_55RPM_30SCFM_TO_COMPARE_STANDARD_INSERT

TIME PURG_TC2 PurgePSI PurgeCFM DEPTH(IN) DwnFrc(lb) RPM Inches/Min

$\begin{array}{lllllllc}17: 09: 27: 67 & 22 & -0.4 & -0.5 & -0.03 & -4 & 55.9 & -0.81 \\ 17: 09: 28: 66 & 22 & 12.4 & 50.8 & 0.61 & 2 & 55.9 & -0.81 \\ 17: 09: 29: 64 & 22 & 21.6 & 53.7 & 1.44 & 1 & 55.6 & 9.16 \\ 17: 09: 30: 63 & 22 & 21.6 & 53.7 & 2.28 & 3 & 55.6 & 9.16 \\ 17: 09: 31: 62 & 22 & 21.8 & 53.7 & 3.10 & 8 & 55.0 & 13.56 \\ 17: 09: 32: 61 & 22 & 21.8 & 53.7 & 3.91 & 13 & 54.8 & 13.56 \\ 17: 09: 33: 60 & 22 & 21.8 & 53.7 & 4.74 & 20 & 54.8 & 13.85 \\ 17: 09: 34: 59 & 22 & 22.0 & 53.7 & 5.56 & 19 & 54.8 & 13.85 \\ 17: 09: 35: 58 & 22 & 22.0 & 53.7 & 6.40 & 15 & 54.7 & 13.47 \\ 17: 09: 36: 56 & 22 & 22.0 & 53.7 & 7.18 & 15 & 54.7 & 13.47 \\ 17: 09: 37: 55 & 22 & 22.4 & 53.7 & 7.98 & 13 & 54.6 & 13.65 \\ 17: 09: 38: 54 & 22 & 22.8 & 53.7 & 8.78 & 26 & 54.8 & 13.65 \\ 17: 09: 39: 53 & 22 & 23.5 & 53.7 & 9.56 & 40 & 54.8 & 12.20 \\ 17: 09: 40: 52 & 22 & 24.1 & 53.6 & 10.36 & 51 & 54.9 & 12.20 \\ 17: 09: 41: 51 & 22 & 24.9 & 53.2 & 11.10 & 55 & 54.9 & 10.37 \\ 17: 09: 42: 50 & 22 & 25.1 & 52.9 & 11.91 & 66 & 54.9 & 10.37 \\ 17: 09: 43: 49 & 22 & 25.3 & 52.8 & 12.64 & 69 & 55.0 & 8.90 \\ 17: 09: 44: 47 & 22 & 25.1 & 52.8 & 13.41 & 59 & 54.9 & 8.90 \\ 17: 09: 45: 46 & 22 & 24.3 & 52.7 & 14.19 & 37 & 54.9 & 13.73 \\ 17: 09: 46: 45 & 22 & 23.5 & 52.7 & 15.02 & 18 & 54.7 & 14.48 \\ 17: 09: 47: 44 & 22 & 22.8 & 52.8 & 15.82 & 10 & 54.8 & 14.48 \\ 17: 09: 48: 43 & 22 & 22.9 & 52.9 & 16.58 & 11 & 54.8 & 14.38 \\ 17: 09: 49: 42 & 22 & 22.8 & 53.0 & 17.38 & 10 & 54.8 & 14.38 \\ 17: 09: 50: 41 & 22 & 22.6 & 53.1 & 18.20 & 5 & 54.8 & 14.15 \\ 17: 09: 51: 39 & 22 & 22.4 & 53.1 & 18.98 & 5 & 54.8 & 14.15\end{array}$


Z10772.TXT

HNF-3557

Rev. 0

100IVD/8_IN_WET_SAND_SN_Z10772_750LB_55RPM_30SCFM_TO_COMPARE_NO_FLOW_INSERT

TIME PURG_TC2 PurgePSI PurgeCFM DEPTH(IN) DwnFrc(Ib) RPM Inches/Min

$\begin{array}{llllllll}17: 47: 49: 37 & 21 & -0.6 & -5.5 & -0.00 & -6 & 55.8 & -1.07 \\ 17: 47: 50: 36 & 21 & -0.6 & -5.5 & -0.02 & -7 & 55.8 & -1.07 \\ 17: 47: 51: 35 & 21 & -0.6 & -5.5 & -0.00 & -7 & 55.8 & -0.17 \\ 17: 47: 52: 34 & 21 & -0.6 & -5.5 & 0.75 & -1 & 55.8 & -0.17 \\ 17: 47: 53: 33 & 21 & 10.3 & 53.7 & 1.53 & 28 & 55.3 & 10.43 \\ 17: 47: 54: 32 & 22 & 25.5 & 50.8 & 2.33 & 18 & 55.3 & 10.43 \\ 17: 47: 55: 31 & 22 & 23.5 & 51.5 & 3.13 & 5 & 54.9 & 13.38 \\ 17: 47: 56: 30 & 21 & 23.3 & 51.8 & 3.91 & 14 & 54.9 & 13.38 \\ 17: 47: 57: 28 & 21 & 23.3 & 51.9 & 4.73 & 13 & 54.9 & 13.20 \\ 17: 47: 58: 27 & 21 & 23.3 & 52.0 & 5.50 & 16 & 54.9 & 13.20 \\ 17: 47: 59: 26 & 21 & 23.3 & 52.0 & 6.33 & 13 & 54.8 & 14.11 \\ 17: 48: 00: 25 & 21 & 23.3 & 52.1 & 7.12 & 31 & 54.8 & 14.11 \\ 17: 48: 01: 24 & 21 & 23.3 & 52.1 & 7.89 & 30 & 54.8 & 13.51 \\ 17: 48: 02: 23 & 22 & 23.5 & 52.1 & 8.67 & 35 & 54.8 & 13.51 \\ 17: 48: 03: 22 & 21 & 23.5 & 52.1 & 9.43 & 38 & 54.8 & 11.65 \\ 17: 48: 04: 20 & 21 & 23.5 & 52.2 & 10.22 & 29 & 54.8 & 11.65 \\ 17: 48: 05: 19 & 21 & 23.7 & 52.2 & 10.97 & 28 & 54.9 & 10.28 \\ 17: 48: 06: 18 & 21 & 23.7 & 52.2 & 11.73 & 33 & 54.9 & 10.28 \\ 17: 48: 07: 17 & 21 & 24.1 & 52.2 & 12.50 & 34 & 54.8 & 8.49 \\ 17: 48: 08: 16 & 21 & 24.5 & 52.2 & 13.31 & 20 & 54.8 & 8.49 \\ 17: 48: 09: 15 & 21 & 24.3 & 52.2 & 14.10 & 16 & 54.8 & 13.54 \\ 17: 48: 10: 14 & 21 & 24.5 & 52.2 & 14.86 & 16 & 54.7 & 13.54 \\ 17: 48: 11: 12 & 21 & 24.3 & 52.2 & 15.65 & 10 & 54.8 & 14.21 \\ 17: 48: 12: 11 & 21 & 24.1 & 52.2 & 16.42 & 10 & 54.8 & 14.21 \\ 17: 48: 13: 10 & 22 & 23.7 & 52.2 & 17.21 & 5 & 54.8 & 14.74 \\ 17: 48: 14: 09 & 21 & 23.5 & 52.2 & 18.00 & 1 & 54.8 & 14.74 \\ 17: 48: 15: 08 & 21 & 23.5 & 52.2 & 18.78 & 5 & 55.0 & 14.27 \\ 17: 48: 16: 07 & 21 & 23.3 & 52.2 & 18.98 & -1 & 55.0 & 9.83 \\ 17: 48: 17: 06 & 21 & 3.1 & -5.8 & 18.96 & -8 & 55: 0 & 9.83 \\ 17: 48: 18: 05 & 21 & -0.8 & -5.8 & 18.97 & -17 & 55.4 & -0.45\end{array}$

PAGE 83 
AA10772.TXT

HNF-3557

Rev. 0

100IVD/8_IN_WET_SAND_SN_AA10772_750LB_55RPM_30SCFM_TO_COMPARE_NO_FLOW_INSERT

TIME PURG_TC2 PurgePSI PurgeCFM DEPTH(IN) DwnFrc(lb) RPM Inches/Min

$\begin{array}{lccccccc}17: 57: 09: 39 & 21 & -0.8 & -5.7 & 0.15 & -6 & 55.4 & -0.52 \\ 17: 57: 10: 33 & 22 & 6.8 & 54.7 & 0.53 & -6 & 55.8 & -0.40 \\ 17: 57: 11: 32 & 22 & 22.8 & 54.3 & 1.36 & 3 & 55.7 & -0.40 \\ 17: 57: 12: 31 & 22 & 22.8 & 54.0 & 2.18 & 9 & 55.7 & 13.30 \\ 17: 57: 13: 29 & 22 & 22.8 & 54.0 & 3.01 & 13 & 55.0 & 13.30 \\ 17: 57: 14: 28 & 22 & 22.8 & 53.9 & 3.82 & 11 & 54.9 & 13.56 \\ 17: 57: 15: 27 & 22 & 22.8 & 53.8 & 4.65 & 16 & 54.9 & 13.56 \\ 17: 57: 16: 26 & 22 & 22.8 & 53.8 & 5.42 & 15 & 54.9 & 13.75 \\ 17: 57: 17: 25 & 22 & 22.6 & 53.8 & 6.27 & 12 & 55.0 & 13.75 \\ 17: 57: 18: 24 & 22 & 22.6 & 53.8 & 7.07 & 10 & 55.0 & 13.94 \\ 17: 57: 19: 23 & 22 & 22.9 & 53.7 & 7.88 & 10 & 55.1 & 13.94 \\ 17: 57: 20: 22 & 22 & 22.9 & 53.6 & 8.69 & 13 & 55.1 & 13.46 \\ 17: 57: 21: 20 & 22 & 22.9 & 53.6 & 9.48 & 11 & 55.1 & 13.46 \\ 17: 57: 22: 19 & 22 & 22.9 & 53.6 & 10.31 & 15 & 55.1 & 12.20 \\ 17: 57: 23: 18 & 22 & 23.3 & 53.5 & 11.09 & 20 & 55.0 & 12.20 \\ 17: 57: 24: 17 & 22 & 24.3 & 53.0 & 11.88 & 38 & 55.0 & 9.80 \\ 17: 57: 25: 16 & 22 & 24.7 & 52.7 & 12.64 & 39 & 55.0 & 9.80 \\ 17: 57: 26: 15 & 22 & 24.5 & 52.6 & 13.40 & 41 & 55.0 & 12.21 \\ 17: 57: 27: 14 & 22 & 24.5 & 52.5 & 14.23 & 35 & 55.0 & 13.59 \\ 17: 57: 28: 12 & 22 & 24.1 & 52.5 & 14.99 & 23 & 55.0 & 13.59 \\ 17: 57: 29: 11 & 22 & 24.1 & 52.5 & 15.78 & 23 & 55.1 & 14.56 \\ 17: 57: 30: 10 & 22 & 23.9 & 52.5 & 16.57 & 15 & 55.1 & 14.56 \\ 17: 57: 31: 09 & 22 & 23.1 & 52.4 & 17.34 & 9 & 55.1 & 14.59 \\ 17: 57: 32: 08 & 22 & 23.1 & 52.4 & 18.14 & 8 & 55.1 & 14.59 \\ 17: 57: 33: 07 & 22 & 22.9 & 52.4 & 18.93 & 7 & 55.1 & 15.07 \\ 17: 57: 34: 06 & 22 & 22.9 & 52.5 & 19.67 & 11 & 55.1 & 15.07\end{array}$


100IVD/9_IN_WET_SAND_SN_Y_NFI_750LB_55RPM_30SCFM_TO_COMPARE_STANDARD_INSERT

TIME PURG_TC2 PurgePSI PurgeCFM DEPTH(IN) DwnFrc(Ib) RPM Inches/Min

$\begin{array}{llllllll}18: 09: 42: 15 & 22 & -0.8 & -5.7 & -0.00 & -6 & 51.2 & -0.62 \\ 18: 09: 43: 14 & 22 & 3.3 & 54.0 & 0.61 & -6 & 52.9 & -0.40 \\ 18: 09: 44: 13 & 22 & 22.0 & 54.7 & 1.45 & -1 & 53.7 & -0.40 \\ 18: 09: 45: 11 & 22 & 22.0 & & & & & \end{array}$

Data lost for remainder of test. Problem with power supply to DAS. 
100IVD/9_IN_WET_SAND_SN_Z_NFI_750LB_55RPM_30SCFM_TO_COMPARE_STANDARD_INSERT

TIME PURG_TC2 PurgePSI PurgeCFM DEPTH(IN) DwnFrc(Ib) RPM Inches/Min

$\begin{array}{lllllllc}18: 19: 13: 54 & 22 & -0.6 & -5.7 & -0.02 & -5 & 55.9 & -0.51 \\ 18: 19: 14: 53 & 22 & 22.0 & 55.9 & 0.70 & 3 & 56.0 & -0.51 \\ 18: 19: 15: 52 & 22 & 22.4 & 55.8 & 1.53 & 1 & 56.0 & 5.13 \\ 18: 19: 16: 50 & 22 & 22.0 & 55.7 & 2.34 & 1 & 55.5 & 14.14 \\ 18: 19: 17: 49 & 22 & 22.0 & 55.6 & 3.17 & 3 & 55.1 & 14.14 \\ 18: 19: 18: 48 & 22 & 22.0 & 55.6 & 3.99 & 4 & 55.1 & 14.22 \\ 18: 19: 19: 47 & 22 & 22.0 & 55.6 & 4.78 & 4 & 55.3 & 14.22 \\ 18: 19: 20: 46 & 22 & 22.2 & 55.5 & 5.61 & 8 & 55.4 & 14.38 \\ 18: 19: 21: 45 & 22 & 22.0 & 55.5 & 6.42 & 7 & 55.4 & 14.38 \\ 18: 19: 22: 44 & 22 & 22.0 & 55.5 & 7.21 & 11 & 55.4 & 14.41 \\ 18: 19: 23: 43 & 22 & 22.4 & 55.4 & 8.04 & 14 & 55.5 & 14.41 \\ 18: 19: 24: 41 & 22 & 22.6 & 55.4 & 8.82 & 18 & 55.5 & 13.69 \\ 18: 19: 25: 40 & 22 & 23.9 & 54.2 & 9.60 & 43 & 55.4 & 13.69 \\ 18: 19: 26: 39 & 22 & 24.7 & 53.4 & 10.39 & 53 & 55.4 & 10.44 \\ 18: 19: 27: 38 & 22 & 25.3 & 52.9 & 11.15 & 70 & 55.4 & 10.44 \\ 18: 19: 28: 37 & 22 & 25.7 & 52.7 & 11.88 & 81 & 55.3 & 9.04 \\ 18: 19: 29: 36 & 22 & 25.9 & 52.7 & 12.64 & 86 & 55.2 & 9.04 \\ 18: 19: 30: 35 & 22 & 26.1 & 52.5 & 13.37 & 86 & 55.2 & 12.28 \\ 18: 19: 31: 33 & 22 & 22.8 & 52.7 & 14.17 & 11 & 55.1 & 12.28 \\ 18: 19: 32: 32 & 22 & 22.4 & 53.0 & 14.99 & 10 & 55.1 & 13.94 \\ 18: 19: 33: 31 & 22 & 22.6 & 53.1 & 15.77 & 13 & 55.1 & 13.94 \\ 18: 19: 34: 30 & 22 & 22.8 & 53.1 & 16.53 & 13 & 55.0 & 13.91 \\ 18: 19: 35: 29 & 22 & 22.6 & 53.2 & 17.35 & 13 & 55.0 & 13.91 \\ 18: 19: 36: 28 & 22 & 22.6 & 53.3 & 18.15 & 10 & 55.0 & 15.18 \\ 18: 19: 37: 27 & 22 & 22.6 & 53.3 & 18.96 & 10 & 54.9 & 15.18\end{array}$


100IVD/9_IN_CRYST_UREA_SN_AA_NFI_750LB_55RPM_30SCFM_TO_COMPARE_STNDARD_INSRT

TIME PURG_TC2 PurgePSI PurgeCFM DEPTH(IN) DwnFrc(lb) RPM Inches/Min

$\begin{array}{lllllllc}\text { 19:03:03:59 } & 21 & -0.6 & 37.3 & 0.21 & 4 & 55.5 & -0.42 \\ \text { 19:03:04:58 } & 22 & 20.8 & 56.2 & 1.01 & 7 & 55.5 & 2.09 \\ \text { 19:03:05:57 } & 22 & 21.4 & 55.8 & 1.84 & 4 & 55.3 & 2.09 \\ \text { 19:03:06:56 } & 22 & 21.4 & 55.8 & 2.63 & 6 & 54.7 & 13.29 \\ \text { 19:03:07:55 } & 22 & 21.4 & 55.7 & 3.46 & 6 & 54.7 & 14.11 \\ \text { 19:03:08:53 } & 21 & 21.4 & 55.7 & 4.27 & 6 & 54.7 & 14.11 \\ \text { 19:03:09:52 } & 21 & 21.2 & 55.6 & 5.10 & 8 & 54.7 & 13.15 \\ 19: 03: 10: 51 & 22 & 21.2 & 55.6 & 5.92 & 5 & 54.7 & 13.15 \\ 19: 03: 11: 50 & 21 & 21.4 & 55.5 & 6.71 & 4 & 54.6 & 13.16 \\ 19: 03: 12: 49 & 21 & 21.4 & 55.5 & 7.53 & 5 & 54.6 & 13.16 \\ 19: 03: 13: 48 & 21 & 21.4 & 55.5 & 8.33 & 6 & 54.6 & 11.28 \\ 19: 03: 14: 47 & 21 & 21.4 & 55.4 & 9.16 & 8 & 54.7 & 11.28 \\ 19: 03: 15: 45 & 21 & 21.4 & 55.4 & 9.94 & 9 & 54.8 & 9.20 \\ 19: 03: 16: 44 & 21 & 21.4 & 55.3 & 10.74 & 8 & 54.8 & 9.20 \\ 19: 03: 17: 43 & 21 & 21.4 & 55.2 & 11.53 & 6 & 54.8 & 10.22 \\ 19: 03: 18: 42 & 21 & 21.4 & 55.2 & 12.35 & 7 & 54.9 & 10.22 \\ 19: 03: 19: 41 & 21 & 21.4 & 55.2 & 13.14 & 6 & 54.9 & 14.05 \\ 19: 03: 20: 40 & 21 & 21.4 & 55.2 & 13.95 & 8 & 54.9 & 14.05 \\ 19: 03: 21: 39 & 21 & 21.6 & 55.2 & 14.76 & 12 & 54.9 & 12.90 \\ 19: 03: 22: 38 & 21 & 21.4 & 55.0 & 15.53 & 10 & 54.9 & 12.90 \\ 19: 03: 23: 36 & 21 & 21.6 & 55.0 & 16.32 & 7 & 54.9 & 13.44 \\ 19: 03: 24: 35 & 21 & 21.4 & 54.8 & 17.14 & 8 & 55.0 & 13.44 \\ 19: 03: 25: 34 & 21 & 21.4 & 54.8 & 17.89 & 10 & 55.0 & 14.17 \\ 19: 03: 26: 33 & 21 & 21.4 & 54.8 & 18.66 & 10 & 54.9 & 14.17\end{array}$




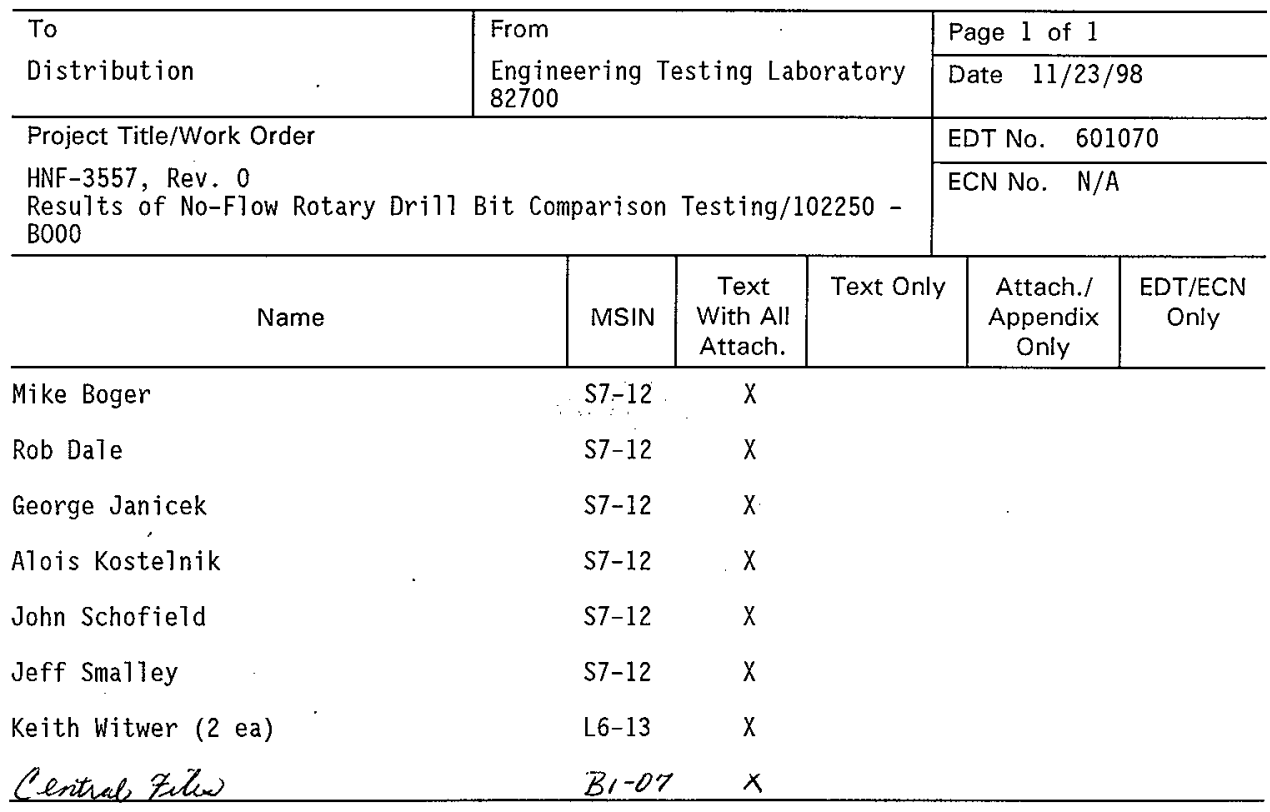

